\title{
Dynamics of a Single Species under Periodic Habitat Fluctuations and Allee Effect
}

\section{Fatma Bayramoğlu Rizaner}

\author{
Submitted to the \\ Institute of Graduate Studies and Research \\ in partial fulfillment of the requirements for the Degree of
}

\author{
Doctor of Philosophy \\ in \\ Mathematics
}

Eastern Mediterranean University

August 2012

Gazimağusa, North Cyprus 
Approval of the Institute of Graduate Studies and Research

Prof. Dr. Elvan Y1lmaz Director

I certify that this thesis satisfies the requirements as a thesis for the degree of Doctor of Philosophy in Mathematics.

Prof. Dr. Nazım Mahmudov

Chair, Department of Mathematics

We certify that we have read this thesis and that in our opinion it is fully adequate in scope and quality as a thesis for the degree of Doctor of Philosophy in Mathematics.

Assoc. Prof. Dr. Svitlana P. Rogovchenko Supervisor

Examining Committee

1. Prof. Dr. Albert Erkip

2. Assoc. Prof. Dr. Sonuç Zorlu Oğurlu

3. Assoc. Prof. Dr. Svitlana P. Rogovchenko 


\section{ABSTRACT}

The dynamics of a single species and harvested single species that goes extinct when rare, is described by nonlinear differential equations
a) $\dot{N}=r N\left(1-\frac{N}{K}\right)\left(\frac{N}{K}-\frac{A}{K}\right)$
b) $\dot{N}=r N\left(1-\frac{N}{K}\right)\left(\frac{N}{K}-\frac{A}{K}\right)-h N$,

where a parameter $A(0<A<K)$ is associated with the Allee effect, $r$ is the intrinsic growth rate, $h$ is the harvesting and $K$ is the carrying capacity of the environment. The intention of this thesis is to study the existence of periodic solutions and their stability properties assuming that $r, A, h$ and $K$ are continuous $T$ - periodic functions. Using rather elementary techniques, we completely describe populations dynamics analyzing influences of both strong $(A>0)$ and weak $(A<0)$ Allee effects. We discuss the effect of harvesting on a single species population in a fluctuating environment whose dynamics is described by a nonlinear differential equation. We consider separately cases of harvesting $(h>0)$ (stocking $(h<0)$ ), weak Allee effect $(A \leq 0)$ and strong Allee effect $(A>0)$. Thus, we answer questions regarding the location of positive periodic solutions and their stability complementing the research in a recent paper by Padhi [14]. Bounds for periodic solutions and estimates for backward blow-up times are also established. Furthermore, we demonstrate advantages of our approach on simple examples to which the results in the cited paper fail to apply.

Keywords: Nonlinear differential equation, Allee effect, periodic solutions, stability, blow up, existence, positive solutions, harvesting. 


\section{$\ddot{O} \mathbf{Z}$}

Yetersiz nüfus yoğunluğundan dolayı soyu tükenmekte olan tek bir türün ve hasat edilen tek bir türün dinamikleri doğrusal olmayan aşağıdaki diferansiyel denklemlerle tanımlanabilir,
a) $\dot{N}=r N\left(1-\frac{N}{K}\right)\left(\frac{N}{K}-\frac{A}{K}\right)$
b) $\dot{N}=r N\left(1-\frac{N}{K}\right)\left(\frac{N}{K}-\frac{A}{K}\right)-h N$.

Burada, $A$ parametresi $(0<A<K)$ Allee etkisi ile ilişkilidir, $r$ içsel büyüme oranı, $h$ hasat kaldırma ve $K$ çevrenin taşıma kapasitesidir. Bu tezin amacı $r, A, h$ ve $K$ 'nin sürekli $T$ - periyodik fonksiyonlar oldukları koşullarda, periyodik çözümlerin varlığını ve onların denge özelliklerini araştırmaktır. Temel teknikler kullanarak güçlü $(A>0)$ ve zayıf $(A<0)$ Allee etkileri incelenerek nüfus dinamikleri tamamıyla elde edilmişlerdir. Dinamikleri doğrusal olmayan diferansiyel denklemlerle tanımlanan dalgalanma ortamındaki tek bir nüfusun hasatı incelenmemiştir. Bu durumda ayrı ayrı hasat $(h>0)$ (stok $(h<0))$, zayıf Allee etkisi $(A \leq 0)$ ve güçlü Allee etkisi $(A>0)$ dikkate alınmıştır. Bu araştırmayla Padhi'nin makalesinde [14] ortaya çıkan pozitif periyodik çözümlerin konumları ve bunların istikrarlarıyla ilgili soruları aydınlattık. Periyodik çözümlerin sınırları ve geri darbe süreleri de tanımlanmıştır. Ayrıca, bu çalışmada önerilen yaklaşımın avantajı Padhi'nin makalesinde [14] önerdiği sonuçların uygulanmayacağı basit örnekler yardımıyla gösterilmiştir.

Anahtar Kelimeler: Doğrusal olmayan diferansiyel denklem, Allee etkisi, periyodik çözümler, çözümün varlı̆̆g, kararlılık, darbe süreleri, pozitif çözümler, hasat. 
To My Family 


\section{ACKNOWLEDGMENTS}

First of all, I would like to thank my supervisor, Assoc. Prof. Dr. Svitlana P. Rogovchenko, for her supervision, continuous support, help, suggestions, unlimited patience and encouragement during my Ph.D. period.

Then, I would like to thank Prof. Dr. Nazım I. Mahmudov, Head of Department of Mathematics, Eastern Mediterranean University, for his support in the department. Also, I am thankful to Prof. Dr. Yuri Rogovchenko of the Umeå University in Sweden for his help of my study. I sincere gratitude to Dr. Mustafa Hasanbulli of the New Zelland Institute for Advanced Study for his valuable help of my figures with Mathematica and for our valuable discussions.

Then, I am thankful to Prof. Dr. Albert Erkip and Assoc. Prof. Dr. Sonuç Zorlu Oğurlu for attending my presentation.

Finally, I would like to give special and infinite thanks to my daughter Ela Rizaner, my husband Ahmet Rizaner and my family Akay Bayramoğlu, Ayşe Bayramoğlu, Hüsnü Bayramoğlu and Gülfere Bayramoğlu for their endless love, care, support and patience. 


\section{TABLE OF CONTENTS}

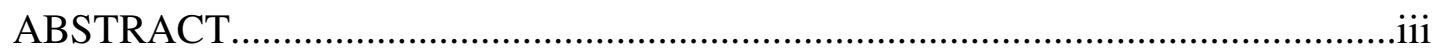

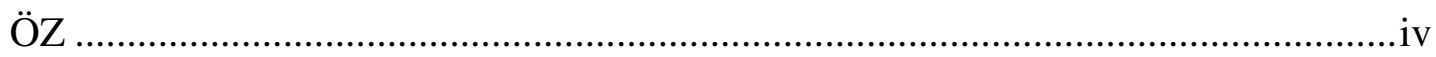

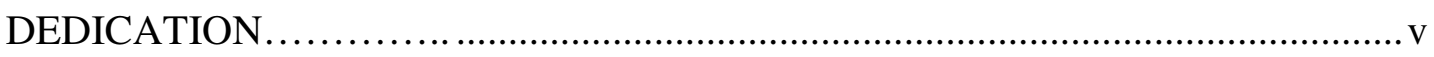

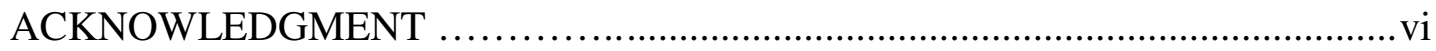

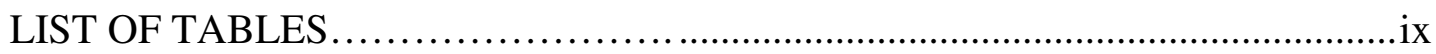

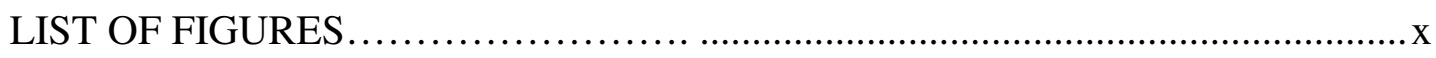

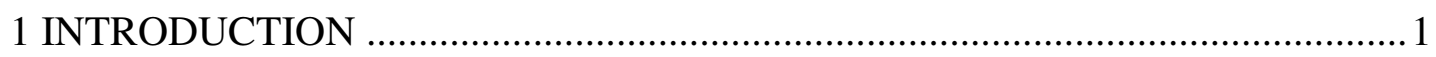

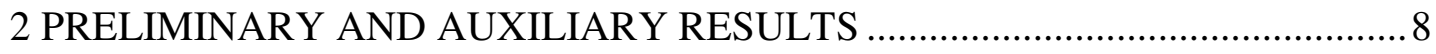

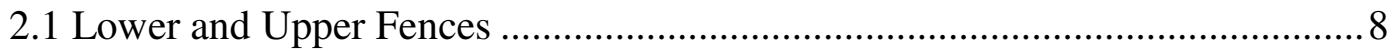

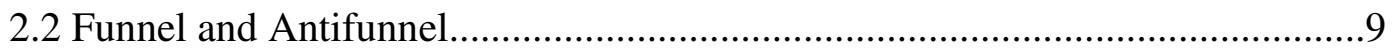

2.3 $T$ - Periodic Solutions and their Asymptotic Behavior..........................

3 EXISTENCE OF THE PERIODIC SOLUTIONS OF THE DIFFERENTIAL

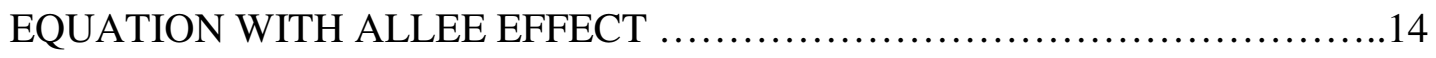

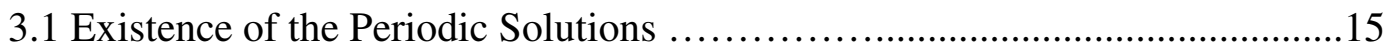

3.1.1 Existence of Periodic Solutions and their Stability for $A(t)>0$ .15

3.1.2 Existence of Periodic Solutions and their Stability for $A(t)=0$

3.1.3 Existence of Periodic Solutions and their Stability for $A(t)<0$ .21

3.2 Blow Up Time. .24 
4 EXISTENCE OF THE PERIODIC SOLUTIONS OF THE DIFFERENTIAL EQUATION WITH ALLEE EFFECT UNDER HARVESTING..................40

4.1 Existence of the Periodic Solutions .................................42

4.1.1 Existence of Periodic Solutions and their Stability for $A(t)>0$ and $h(t)>0$.

4.1.2 Existence of Periodic Solutions and their Stability for $A(t)>0$ and $h(t)<0$.

4.1.3 Existence of Periodic Solutions and their Stability for $A(t)<0$ and $h(t)>0$. .50

4.1.4 Existence of Periodic Solutions and their Stability for $A(t)<0$ and $h(t)<0$.

4.1.5 Existence of Periodic Solutions and their Stability for $A(t)=0, h(t)>0$ and $A(t)=0, h(t)<0$. 55

4.2 Examples and Discussion. .59

5 CONCLUSION. . .74

REFERENCES. .85 


\section{LIST OF TABLES}

Table 1: Phenomenological models of demographic Allee effect and harvesting (or stocking) for the per capita growth rate $g(N)=r\left(1-\frac{N}{K}\right)\left(\frac{N}{K}-\frac{A}{K}\right) \ldots \ldots \ldots \ldots \ldots \ldots \ldots . . .82$ 


\section{LIST OF FIGURES}

Figure 1: Three periodic solutions and several other solutions to Eq. (3.21) for $\gamma=1$

Figure 2: Vertical asymptotes (green), upper (orange) and lower (lilac) solutions along with several other solutions (red) to Eq. (3.21) for $\gamma=1$

Figure 3: Two periodic solutions and several other solutions to Eq. (3.21) for $\gamma=0$

Figure 4: Three periodic solutions and several other solutions to Eq. (3.21) for $\gamma=-1$

Figure 5: Existence of one positive asymptotically stable and one positive unstable periodic solution of Eq. (4.8) in case of strong Allee effect for $\gamma_{1}=1$ and harvesting for $\gamma_{2}=1$

Figure 6(a): Existence of one positive asymptotically stable and one positive unstable periodic solution of Eq. (4.9) in case of strong Allee effect $\gamma_{1}=1$ and stocking $\gamma_{2}=-1$ .65

Figure 6(b): Existence of one positive asymptotically stable periodic solution of Eq. (4.10) in case of strong Allee effect $\gamma_{1}=1$ and stocking $\gamma_{2}=-1$ .65

Figure 7(a): Existence of one positive asymptotically stable and one positive unstable periodic solution of Eq. (4.11) in case of weak Allee effect $\gamma_{1}=-1$ and harvesting $\gamma_{2}=1$ 
Figure 7(b) Existence of one positive asymptotically stable of Eq. (4.12) in case of

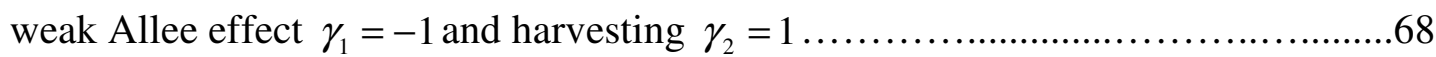

Figure 8: Existence of one positive asymptotically stable periodic solution of Eq (4.13) in case of weak Allee effect $\gamma_{1}=-1$ and stocking $\gamma_{2}=-1$ . .70

Figure 9(a): Existence of one positive asymptotically stable and one positive unstable periodic solution of Eq. (4.14) in case of harvesting $\gamma_{1}=0, \gamma_{2}=1$ 73 Figure 9(b): Existence of one positive asymptotically stable of Eq. (4.15) in case of stocking $\gamma_{1}=0, \gamma_{2}=-1$ 73

Figure 10: Qualitative changes in the dynamics of Eq. (3.21) as $\gamma$ changes from 1 to -1 with the step -0.25 . .76 Figure 11: Qualitative changes in the dynamics of Eqs. (4.8) - (4.15) as $\gamma_{1}$ and $\gamma_{2}$ change from 1 to -1 with the step -0.25 . .80 


\section{Chapter 1}

\section{INTRODUCTION}

The dynamics of a single non-structured population is directly influenced by regular changes in environmental conditions such as climate, food availability, predator scarcity, etc. Therefore, seasonal habitat fluctuations should be preferably taken into consideration in mathematical models due to the significant effect they have on the population density, even during brief periods when the physical and biological environments remain nearly constant. In fact, Nicholson [1] disputed that any periodic change of climate tends to impose its period upon oscillations of internal origin or to cause such oscillations to have a harmonic relation to periodic climate changes. Many researchers emphasized particular importance of periodic and almost periodic fluctuations in mathematical biology. For instance, Vance and Coddington [2] pointed out that periodic time variation holds considerable promise as a means to explore time-varying ecological processes.

Although rapid progress in mathematical biology within the last few decades led to incorporation of time-varying parameters in many models, the effect of environmental fluctuations is still being quite often underestimated or even neglected. Henson and Cushing [3] stressed that fluctuating environments are of particular interest to population biologists. Despite this fact, the vast majority of mathematical models used in population dynamics and ecology are autonomous and assume a constant environment. As a result, virtually all fundamental principles in 
theoretical population dynamics are based upon the assumption of a constant environment: monotonic logistic growth, competitive exclusion and ecological niche, predator-prey oscillations, and so on.

In the cited paper, the authors obtained very interesting results that provide the first rigorous evidence, via model analysis of laboratory data, that an effective periodicity can have a positive effect on total population biomass. This positive development is in line with the prediction made by Brauer and Sánchez [4] who emphasized that a general theory of the qualitative behavior of periodic population models, both single species and interacting species, would have many applications. Development of such theory appears to be not an easy task, as the discussion for a relatively simple logistic model in [5] demonstrates, see also the references cited therein.

In Chapter 2, we give some basic definitions and theorems about $T$ - periodic solutions and their asymptotic behavior, lower and upper fences, funnel and antifunnel.

In this thesis, we are concerned with a nonlinear differential equation [42],

$$
\dot{N}(t)=r(t) N(t)\left(1-\frac{N(t)}{K(t)}\right)\left(\frac{N(t)}{K(t)}-\frac{A(t)}{K(t)}\right)
$$

with continuous, positive $T$-periodic functions $r(t), K(t)$ and a continuous $T$ periodic function $A(t)$. Eq. (1.1) describes the dynamics of a single species subject to Allee effect, where the case of constant coefficients is dealt with [6]. This equation can be also written in a compact form

$$
\dot{N}(t)=N(t) g(N(t))
$$

where 


$$
g(N(t))=r(t)\left(1-\frac{N(t)}{K(t)}\right)\left(\frac{N(t)}{K(t)}-\frac{A(t)}{K(t)}\right)
$$

In Eqs. (1.1) and (1.2), $N$ denotes the population size, the function $g(N)$ stands for the density-dependent per capita growth rate, $r(t)$ denotes the maximum per capita population growth rate without Allee effect, $A(t)$ is the Allee threshold, that is, a critical population size or density below which the per capita population growth rate becomes negative, $K(t)$ is the carrying capacity of the environment.

In Chapter 3, we investigate the existence of periodic solutions of Eq. (1.1) and their asymptotic behavior for the Allee threshold, $A(t)>0, A(t)=0, A(t)<0$. A so called Allee effect occurs when positive density dependence dominates at low population size; for $A>0$ (strong Allee effect), it characterizes the dynamics of a single population that goes extinct when rare. This effect is often caused by difficulties in mate finding; it may also depend, as indicated by Lewis and Kareiva [7], on other factors such as inbreeding depression, food exploitation, predator avoidance of defense, etc. Recent studies indicate that a strong Allee effect $(A>0)$ can give rise to a complex dynamics even in simple models arising in mathematical ecology and epidemiology; it can lead to critical population size or population levels below which the population crashes to extinction. Courchamp [10] pointed out that studies demonstrating Allee effects and determining their causal mechanisms, either theoretically or empirically, ought to be more numerous in the future. For more information on the wide variety of Allee effects in mathematical ecology, we refer to 
an excellent monograph by Courchamp [10] and review papers by Boukal and Berec [12] or Berec [13].

Research reported in this thesis has been strongly motivated by a very recent contribution due to Padhi [14] who discussed existence of periodic solutions to a general scalar differential equation

$$
\frac{d x(t)}{d t}=-A(t) x(t)+f(t, x(t)),
$$

where $A$ and $f$ are continuous $T$ - periodic real valued functions on $\mathbb{R}$ and $\mathbb{R}^{2}$ respectively. Using Legget-Williams multiple fixed point theorem [15], Padhi [14] established existence of at least two positive periodic solutions to Eq. (1.3). As an application, a sufficient condition for the existence of periodic solutions to equation

$$
\frac{d y(t)}{d t}=a(t) y(t)(y(t)-b(t))(c(t)-y(t))
$$

describing the dynamics of a single species subject to Allee effect has been derived. In Eq. (1.4), all coefficients are positive $T$ - periodic functions; it is also assumed that $b(t)<c(t)$, for all $t \in \mathbb{R}$. We formulate one of the principal results obtained in the cited paper.

Theorem 1.1 Let

$$
M=\int_{0}^{T} a(s) c^{2}(s) d s \quad \text { and } \quad L=\int_{0}^{T} a(s) b(s) c(s) d s
$$

If

$$
\begin{gathered}
\frac{M+L+\sqrt{(M+L)^{2}-4 M \exp (-L)(\exp (L)-1)}}{2 M} \\
>\frac{\exp (2 L)-\exp (-L)}{M+L},
\end{gathered}
$$


Eq. (1.4) has at least two positive $T$ - periodic solutions.

Padhi [14] concluded the paper by pointing out the following. It would be interesting to develop results that identify the exact number of positive periodic solutions admitted by the considered model and study their stability nature. Such study becomes imperative from resource management perspective.

In this thesis, we answer the questions raised by Padhi [14] demonstrating that much more information regarding the properties of periodic and, in general, all solutions to Eq. (1.1) can be obtained by combining methods of mathematical analysis with a simple direction field argument and the upper and lower solutions techniques. Our arguments are far from being trivial because the development of a qualitative theory for nonautonomous equations is much more difficult compared to autonomous case and requires, as pointed out by Langa [16], an essentially nonautonomous analysis. In particular, in what regards stability of solutions and properties of attractors, Berger and Siegmund [17] emphasized recently that there exist several non-equivalent definitions for nonautonomous attractors, and in many respects the nonautonomous situation remains fundamentally different from the autonomous one. Although serious difficulties that arise in the study of stability and bifurcation properties of generic nonautonomous differential equations, our elementary yet efficient technique for Eq. (1.1) allows one to determine the exact number of periodic solutions, localize them and describe their stability properties characterizing completely the dynamics of the population.

In this chapter, we examine the blow up in finite time forward or backward, which prompts possibility for existence of vertical asymptotes for solutions. We estimate the behavior of solutions and we use upper and lower solutions to associated differential equations with constant coefficients whose exact solutions can be easily 
obtained in a closed form. We explore the examples of the asymptotic behavior and vertical asymptotes of the Eq. (3.21) for $\gamma=-1,0,1$ with the figures.

In Chapter 4, we investigate the effect of harvesting on the dynamics of population in a fluctuating environment described by a nonlinear differential equation [43],

$$
\dot{N}(t)=r(t) N(t)\left(1-\frac{N(t)}{K(t)}\right)\left(\frac{N(t)}{K(t)}-\frac{A(t)}{K(t)}\right)-h(t) N(t)
$$

with continuous, positive $T$ - periodic functions $r(t), K(t)$ and a continuous $T$ periodic function $A(t)$ that is,

$$
r(t+T)=r(t), K(t+T)=K(t), \text { and } A(t+T)=A(t) \text { for all } t \in \mathbb{R}
$$

Eq. (1.7) describes the dynamics of a single species subject to Allee effect, where the case of constant coefficients is dealt with [6]. $N$ denotes the population size, $r(t)$ denotes the maximum per capita population growth rate without Allee effect, $A(t)$ is the Allee threshold for a strong Allee effect, that is, a critical population size or density below which the per capita population growth rate becomes negative, $K(t)$ is the carrying capacity of the environment, $h(t)$ is continuous function of harvesting. The dynamics of population in a fluctuating environment described by Eq. (1.7) or its particular cases in the presence of harvesting has been studied by many authors. We would like mention to interesting contributions by Brauer and Sánchez [4], Lazer Chapter 1[38], Lazer and Sánchez [39], [40] and Padhi [14].

In this Chapter, all solutions to Eq. (1.7) are obtained by combining methods of mathematical analysis with simple direction field and the upper and lower solutions techniques. The discussed technique for Eq. (1.7) allows one to determine 
the exact number of periodic solutions, localize them and describe their stability properties characterizing the dynamics of the system for the properties of $A$ and $h$, $(A>0, A<0, A=0, h>0, h<0)$. We explained the existence of periodic solutions for $h=0$ in Chapter 3. We explore the examples for the asymptotic behavior of the Eq. (4.8) - Eq. (4.15) for $\gamma_{1}, \gamma_{2}=-1,0,1$, with the figures. 


\section{Chapter 2}

\section{PRELIMINARY AND AUXILIARY RESULTS}

In this chapter, we collected relevant results that are used in the sequel. In the qualitative theory of differential equations, one often uses lower and upper solutions, also called lower and upper fences (subsolutions and supersolutions) to prove existence and uniqueness of solutions, to describe their local and global behavior, to provide efficient estimates for solutions, etc.

\subsection{Lower and Upper Fences}

Definition 2.1.1 For the differential equation

$$
\frac{d x(t)}{d t}=f(t, x(t))
$$

a continuously differentiable function $\alpha(t)$ is called a lower fence (lower solution)

if $\dot{\alpha}(t) \leq f(t, \alpha(t))$ for all $t \in I$, where I is an open or closed interval with the endpoints $a$ and $b, \quad b \leq+\infty, a \geq-\infty$. Similarly, a continuously differentiable function $\beta(t)$ is called an upper fence (upper solution) if $\dot{\beta}(t) \geq f(t, \beta(t))$ for all $t \in I . \quad$ A lower/upper fence (lower/upper solution) is said to be strong (strict) if $\dot{\alpha}(t)<f(t, \alpha(t)) \quad$ or $\dot{\beta}(t)>f(t, \beta(t))$. A lower fence $\alpha(t) \quad$ ( an upper fence $\beta(t)$ ) is called nonporous for the differential equation (2.1) with solution $x(t)$ if $\alpha(t) \leq x(t)$ then $\alpha(t)<x(t)$ or if $\beta(t) \geq x(t)$ then $\beta(t)>x(t)$, for all $t \in I$ where $x(t)$ is defined. 


\subsection{Funnel and Antifunnel}

Definition 2.2.1 Let $\alpha(t)$ and $\beta(t)$ be nonporous lower and upper fences for differential equation (2.1) over I. If $\alpha(t)<\beta(t) \quad(\alpha(t)>\beta(t))$, the set of points $(t, y)$ such that, for all $t \in I, \quad \alpha(t) \leq y \leq \beta(t) \quad(\beta(t) \leq y \leq \alpha(t))$, is called a funnel (antifunnel).

For more details, we refer the reader to the book by Hubbard and West [17] where basic facts regarding fences and funnels can be found.

The following two results regard existence of periodic solutions to Eq. (1.1) and Eq. (1.7); they are very useful for our discussion in the Chapter 3 and Chapter 4. The first one is a celebrated Massera Theorem [19], [20], [21], [22] and the second one is a direct consequence of the Massera Theorem, [4], [20], [23], or [24].

\subsection{T-Periodic Solutions and Their Asymptotic Behavior}

Theorem 2.3.1 Suppose that the function $f(t, x)$ is continuous, satisfies the uniqueness condition for all $t$ and $x$, and

$$
f(t+T, x) \equiv f(t, x)
$$

If a solution $x=\phi(t)$ of a differential equation (2.1) is bounded for all $t \geq t_{0}$ ( for all $t \leq t_{0}$ ), then either it is $T$ - periodic or it asymptotically approaches some $T$ periodic solution as $t \rightarrow+\infty \quad$ (as $t \rightarrow-\infty)$.

Corollary 2.3.2 Assume that $f(t, x)$ is a smooth function satisfying (2.2) and there exist constants $a, b(a<b)$ such that $f(t, b)<0<f(t, a)$ for every $t$. Then there exists a $T$-periodic solution $x=\phi(t)$ of equation (2.1) satisfying $\phi(0)=c_{1}$ for some $c_{1} \in(a, b)$. 
Remark 2.3.3 A $T$-periodic solution $x=\phi(t)$ in Corollary (2.3.2) is asymptotically stable, see Brauer and Sánchez [4]. If, in addition, there exists a constant $d<a$ such that $f(t, d)<0$ for all $t$, there exists a second unstable $T$-periodic solution $x=\psi(t)$ of equation (2.1) satisfying $\psi(0)=c_{2}$ for some $c_{2} \in(d, a)$.

The next theorem was established in a more general form by Nkashama [25] as an extension of the existence result proved by Lakshmikantham and Leela [26] for a periodic boundary value problem

$$
\frac{d x(t)}{d t}=f(t, x(t)), \quad x(0)=x(T)
$$

with a continuous function $f:[0, T] \times \mathbb{R} \rightarrow \mathbb{R}$ to periodic boundary value problems with Carathéodory functions. It serves the same purpose as Theorem (2.3.1) and Corollary (2.3.2) Since our concern is polynomial differential equations of the form (1.1) with continuous coefficients, we assume continuous differentiability of $f$ to simplify the presentation.

Theorem 2.3.4 (i) Let $\alpha(t)$ and $\beta(t)$ be respectively lower and upper solutions for equation (2.1) on $I=[0, T]$ such that $\alpha(t) \leq \beta(t)$ on I. Assume also that

$$
\alpha(0) \leq \alpha(T) \quad \text { and } \quad \beta(0) \geq \beta(T)
$$

Then periodic boundary value problem (2.3) has at least one solution $x^{*}(t)$ satisfying $\alpha(t) \leq x^{*}(t) \leq \beta(t)$ for all $t \in I$.

(ii) Let $\alpha(t)$ and $\beta(t)$ be respectively lower and upper solutions for equation (2.1) on I such that $\alpha(t) \geq \beta(t)$ on I. Assume also that

$$
\alpha(0) \geq \alpha(T) \quad \text { and } \quad \beta(0) \leq \beta(T)
$$


Then periodic boundary value problem (2.3) has at least one solution $x^{*}(t)$ satisfying $\beta(t) \leq x^{*}(t) \leq \alpha(t)$ for all $t \in I$.

Andersen and Sandqvist [27] proved an interesting result providing an upper bound for the number of periodic solutions to Eq. (2.1). We extract it from the cited paper skipping details regarding relationship between the properties of periodic solutions and characteristic exponents that are not relevant to the study undertaken here.

Theorem 2.3.5 Assume that the function $f$ in Eq. (2.1) belongs to $C^{3}\left(\mathbb{R}^{2}\right)$, satisfies Eq. (2.2) and is such that $f_{x x x}(t, x) \leq 0$, for every $(t, x) \in \mathbb{R}^{2}$, and there is $t_{0}$ such that $f_{x x x}\left(t_{0}, x\right)<0$, for every $x \in \mathbb{R}$. Then Eq. (2.1) has at most three periodic solutions.

The following result due to Korman and Ouyang [28] gives the exact number of periodic solutions for a differential equation of the form

$$
\dot{x}=e(t)(x-a(t))(x-b(t))(x-c(t)),
$$

where $a, b, c$, and $e$ are continuous real-valued functions.

Theorem 2.3.6 Let Eq. (2.4) have continuous T-periodic coefficients such that e(t) has constant sign for almost all $t$ and

$$
\max _{t \in \mathbb{R}} a(t)<\min _{t \in \mathbb{R}} b(t), \quad \max _{t \in \mathbb{R}} b(t)<\min _{t \in \mathbb{R}} c(t) .
$$

Then Eq.(2.4) has exactly three T-periodic solutions. 
One can thus deduce from Theorem (2.3.6) the following proposition that provides the exact number of periodic solutions of Eq. (1.1) for the cases $A(t)>0$ (strong Allee effect), $A(t)=0$ and $A(t)<0$ (weak Allee effect).

Theorem 2.3.7 Consider Eq. (1.1) with continuous, positive $T$ - periodic functions $r(t)$ and $K(t)$. Assume also that either $(i) A(t)$ is a continuous, positive $T$ periodic functions such that

$$
\max _{t \in \mathbb{R}} A(t)<\min _{t \in \mathbb{R}} K(t)
$$

or (ii) $A(t)$ is a continuous, non-positive $T$ - periodic function such that

$$
-\min _{t \in \mathbb{R}} A(t)<\min _{t \in \mathbb{R}} K(t)
$$

Then, in either case, Eq. (1.1) has exactly three T-periodic solutions.

Remark 2.3.8 One should note that Theorems (2.3.5) - (2.3.7) fail to hold for generic cubic differential equations. For instance, Lins Neto [29] provided examples of equations of the form

$$
\frac{d y(t)}{d t}=a_{3}(t) y^{3}(t)+a_{2}(t) y^{2}(t)
$$

which have at least $k$ periodic solutions and not all solutions to Eq.(2.5) are periodic, where $k$ is a positive integer and $a_{2}, a_{3}$ are polynomials in $t$ or in $\cos (\pi t)$ and $\sin (\pi t)$. It is a special form of Eq. (1.1) that helps describe its dynamics more precisely.

We conclude this section with a simple technical proposition that facilitates estimates in the proofs of our main results. 
Lemma 2.3.9 Let $f_{1}$ and $f_{2}$ be two continuous real-valued functions defined on some interval $[a, b]=\mathbf{I} \subset \mathbb{R}$.

(i) If $\min _{t \in \mathrm{I}} f_{1}(t) \leq f_{1}(t) \leq \max _{t \in \mathrm{I}} f_{1}(t)<0<\min _{t \in \mathrm{I}} f_{2}(t) \leq f_{2}(t) \leq \max _{t \in \mathrm{I}} f_{2}(t)$,

then

$$
\min _{t \in \mathbf{I}} f_{1}(t) \cdot \max _{t \in \mathbf{I}} f_{2}(t) \leq f_{1}(t) f_{2}(t) \leq \max _{t \in \mathbf{I}} f_{1}(t) \cdot \min _{t \in \mathbf{I}} f_{2}(t) \quad \text { on } \mathbf{I} .
$$

(ii) If $\max _{t \in \mathbf{I}}\left[f_{1}(t), f_{2}(t)\right]<0$,

then

$$
\max _{t \in \mathbf{I}} f_{1}(t) \cdot \max _{t \in \mathbf{I}} f_{2}(t) \leq f_{1}(t) f_{2}(t) \leq \min _{t \in \mathbf{I}} f_{1}(t) \cdot \min _{t \in \mathbf{I}} f_{2}(t) \quad \text { on } \mathbf{I} .
$$




\section{Chapter 3}

\section{EXISTENCE OF PERIODIC SOLUTIONS OF THE DIFFERENTIAL EQUATION WITH ALLEE EFFECT}

In this chapter, we study the existence of periodic solutions to Eq. (1.1) and their stability properties. In what follows, $P_{\max }$ and $P_{\min }$ denote respectively the maximum and the minimum of a continuous periodic function $P(t)$. Taking into account that the exact number of periodic solutions to Eq. (1.1) is established in Theorem (2.3.7) which is a direct consequence of a more general Theorem (2.3.6) of Korman and Ouyang [28] and following the suggestion of Padhi [14], we concentrate our efforts on establishing location and stability properties of positive periodic solutions. When we have a negative periodic solution of the differential equation (1.1) describing dynamics of a population, it has no biological meaning. If the periodic solution is positive and stable then the biological meaning is the population survives and fluctuates periodically.

The solutions to Eq. (1.1) may not be defined for all values of $t$; some may blow up in finite time forward or backward, which prompts possibility for existence of vertical asymptotes for solutions. Direct integration of Eq. (1.1) is not possible despite of its relatively simple structure. Therefore, to estimate the behavior of solutions we use upper and lower solutions to associated differential equations with constant coefficients whose exact solutions can be easily obtained in a closed form. 


\subsection{Existence of Periodic Solutions}

In this section, we study the existence of periodic solutions to Eq. (1.1) and their stability properties. Three cases where the $A(t)$ is positive (strong Allee effect), zero and negative (weak Allee effect) are considered separately.

\subsubsection{Existence of Periodic Solutions and their Stability for $A(t)>0$}

In this subsection, we study the existence of periodic solutions to Eq. (1.1) and their stability properties for the Allee threshold $A(t)$ is positive (strong Allee effect).

Theorem 3.1 (Bistability) Let $A(t)>0$ and assume that

$$
A_{\max }<K_{\min }
$$

Then Eq. (1.1) has three periodic solutions: the asymptotically stable trivial solution $N_{\text {triv }}(t)=0$ and two positive solutions, an asymptotically stable solution $N_{1}(t)$ and an unstable solution $N_{2}(t)$.

Proof. Observe first that

$$
A_{\min } \leq A(t) \leq A_{\max }<\frac{A_{\max }+K_{\min }}{2}<K_{\min } \leq K(t) \leq K_{\max }
$$

Let $M^{*}>K_{\max }$. Then, using Lemma (2.3.9), we conclude that, for all $t \in \mathrm{J}=\left[t_{0}, t_{0}+T\right]$

$$
\begin{aligned}
f\left(t, M^{*}\right) & =\frac{r(t)}{K^{2}(t)} M^{*}\left(K(t)-M^{*}\right)\left(M^{*}-A(t)\right) \\
& \leq \frac{r_{\min }}{K_{\max }^{2}} M^{*}\left(K_{\max }-M^{*}\right)\left(M^{*}-A_{\max }\right)<0 .
\end{aligned}
$$

On the other hand, 


$$
\begin{aligned}
& f\left(t, \frac{A_{\max }+K_{\min }}{2}\right)=\frac{r(t)}{K^{2}(t)}\left(\frac{A_{\max }+K_{\min }}{2}\right)\left(K(t)-\frac{A_{\max }+K_{\min }}{2}\right) \\
& \times\left(\frac{A_{\text {max }}+K_{\min }}{2}-A(t)\right) \geq \frac{r_{\min }}{K_{\text {max }}^{2}}\left(\frac{A_{\text {max }}+K_{\min }}{2}\right) \\
& \times\left(K_{\min }-\frac{A_{\max }+K_{\min }}{2}\right)\left(\frac{A_{\max }+K_{\min }}{2}-A_{\max }\right) \\
& =\frac{r_{\min }}{8 K_{\max }^{2}}\left(A_{\max }+K_{\min }\right)\left(K_{\min }-A_{\max }\right)^{2}>0 .
\end{aligned}
$$

Since

$$
f\left(t, \beta_{1}(t)\right)<\dot{\beta}_{1}(t)=0 \quad \text { and } \quad f\left(t, \alpha_{1}(t)\right)>\dot{\alpha}_{1}(t)=0 \text {, }
$$

for all $t \geq t_{0}$, we conclude that $\beta_{1}(t)=M^{*}$ and $\alpha_{1}(t)=\left(A_{\max }+K_{\min }\right) / 2$ are strict upper and lower fences respectively. Note that $\alpha_{1}(t)<\beta_{1}(t)$. Therefore, there is a funnel that contains an asymptotically stable periodic solution $N_{1}(t)$ to Eq. (1.1). Furthermore, $N_{1}(t)$ attracts all other solutions with close initial data; these solutions stay in the funnel after entering it once. The existence of a periodic solution $N_{1}(t)$ is guaranteed by Theorem (2.3.1), Corollary (2.3.2), or Theorem (2.3.4), as well as corresponding theorems about funnels; see Hubbard and West [18]. Corollary (2.3.2) and theorems on funnels [17] ensure asymptotic stability. Similar reasoning applies in the rest of this proof and for other results included in this section.

Next, observe that

$$
\begin{aligned}
f\left(t, \frac{A_{\min }}{2}\right) & =\frac{r(t)}{K^{2}(t)} \frac{A_{\min }}{2}\left(K(t)-\frac{A_{\min }}{2}\right)\left(\frac{A_{\min }}{2}-A(t)\right) \\
& \leq \frac{r_{\text {min }}}{K_{\text {max }}^{2}} \frac{A_{\min }}{2}\left(K_{\text {min }}-\frac{A_{\min }}{2}\right)\left(\frac{A_{\min }}{2}-A_{\min }\right) \\
& =-\frac{r_{\min } A_{\min }^{2}}{8 K_{\max }^{2}}\left(2 K_{\min }-A_{\min }\right)<0
\end{aligned}
$$

and 


$$
\begin{aligned}
f\left(t,-\frac{A_{\min }}{2}\right) & =\frac{r(t)}{K^{2}(t)}\left(-\frac{A_{\min }}{2}\right)\left(K(t)+\frac{A_{\min }}{2}\right)\left(-\frac{A_{\min }}{2}-A(t)\right) \\
& \geq \frac{r_{\min }}{K_{\max }^{2}}\left(-\frac{A_{\min }}{2}\right)\left(K_{\text {min }}+\frac{A_{\min }}{2}\right)\left(-\frac{A_{\min }}{2}-A_{\min }\right) \\
& =\frac{3 r_{\min } A_{\min }^{2}}{8 K_{\max }^{2}}\left(2 K_{\min }+A_{\min }\right)>0 .
\end{aligned}
$$

The same argument as above leads to the conclusion that $\beta_{0}(t)=A_{\min } / 2$ and $\alpha_{0}(t)=-A_{\min } / 2$ are, respectively, strict upper and lower fences because

$$
f\left(t, \alpha_{0}(t)\right)>\dot{\alpha}_{0}(t)=0, \quad f\left(t, \beta_{0}(t)\right)<\dot{\beta}_{0}(t)=0
$$

Since $\alpha_{0}(t)<\beta_{0}(t)$, the trivial solution $N_{\text {triv }}(t)$ to Eq. (1.1) is located in a funnel formed by $\alpha_{0}$ and $\beta_{0}$. This solution is asymptotically stable; all nearby solutions enter a funnel and stay eventually there.

To study unstable positive periodic solution $N_{2}(t)$, we reverse the time, keeping in mind that a past attractor is a future repellor. Note that a new function $N(\tau)=N(-t)$ satisfies a modified differential equation

$$
\frac{d N(\tau)}{d \tau}=-r(\tau) N(\tau)\left(1-\frac{N(\tau)}{K(\tau)}\right)\left(\frac{N(\tau)}{K(\tau)}-\frac{A(\tau)}{K(\tau)}\right)
$$

Let $\hat{f}(\tau, N)$ denote the right-hand side of Eq. (3.4). Then

$$
\begin{aligned}
\hat{f}\left(\tau, \frac{A_{\max }+K_{\min }}{2}\right) & =-\frac{r(\tau)}{K^{2}(\tau)}\left(\frac{A_{\max }+K_{\min }}{2}\right)\left(K(\tau)-\frac{A_{\max }+K_{\min }}{2}\right) \\
& \times\left(\frac{A_{\text {max }}+K_{\min }}{2}-A(\tau)\right) \leq \frac{r_{\min }}{K_{\max }^{2}}\left(\frac{A_{\text {max }}+K_{\min }}{2}\right) \\
& \times\left(K_{\text {min }}-\frac{A_{\max }+K_{\min }}{2}\right)\left(A_{\max }-\frac{A_{\text {max }}+K_{\min }}{2}\right) \\
& =-\frac{r_{\min }}{8 K_{\max }^{2}}\left(A_{\max }+K_{\min }\right)\left(K_{\min }-A_{\max }\right)^{2}<0
\end{aligned}
$$


and

$$
\begin{aligned}
\hat{f}\left(\tau, \frac{A_{\text {min }}}{2}\right) & =\frac{r(\tau)}{K^{2}(\tau)}\left(\frac{A_{\min }}{2}\right)\left(K(\tau)-\frac{A_{\min }}{2}\right) \\
& \times\left(A(\tau)-\frac{A_{\min }}{2}\right) \geq \frac{r_{\text {min }} A_{\text {min }}^{2}}{8 K_{\max }^{2}}\left(2 K_{\text {min }}-A_{\text {min }}\right)>0 .
\end{aligned}
$$

Reasoning as above, we conclude that $\beta_{2}(t)=\left(A_{\max }+K_{\min }\right) / 2$ and $\alpha_{2}(t)=A_{\min } / 2$ are strict upper and lower fences respectively, since

$$
\hat{f}\left(t, \alpha_{2}(t)\right)>\dot{\alpha}_{2}(t)=0, \quad \hat{f}\left(t, \beta_{2}(t)\right)<\dot{\beta}_{2}(t)=0 .
$$

The fact that $\alpha_{2}(t)<\beta_{2}(t)$ yields that these fences form a funnel as $\tau \rightarrow \infty$ and, correspondingly, an antifunnel as $t \rightarrow \infty$. This means that there exists an asymptotically stable, as $\tau \rightarrow+\infty$, periodic solution to Eq. (3.4) satisfying

$$
\frac{A_{\min }}{2}<N(\tau)<\frac{A_{\max }+K_{\min }}{2}
$$

Consequently, Eq. (1.1) has an unstable periodic solution $N_{2}(t)$, as $t \rightarrow+\infty$, satisfying Eq. (3.5). Therefore, we have established existence of three periodic solutions to Eq. (1.1), namely, the trivial solution $N_{\text {triv }}(t)$ and two positive solutions, a stable solution $N_{1}(t)$ and an unstable solution $N_{2}(t)$ satisfying, for all $t \in \mathbb{R}$

$$
-\frac{A_{\min }}{2}<N_{\text {triv }}(t)<\frac{A_{\min }}{2}<N_{2}(t)<\frac{A_{\max }+K_{\min }}{2}<N_{1}(t)<M^{*} .
$$

By Theorem (2.3.6), Eq. (1.1) cannot have more periodic solutions. The proof is complete. 
In the following proposition, we indicate the corridors where periodic solutions are located. It can be easily proved along the same lines as Theorem (3.1). For instance, one can use $K_{\max }+\varepsilon$ rather than $M^{*}$ to derive an estimate similar to (3.2) and $K_{\min }-\varepsilon$ to obtain an inequality analogous to (3.3).

Corollary 3.2 Let the assumptions of Theorem (3.1) be satisfied. Then, for any $\varepsilon>0$, the following estimates for the three periodic solutions of Eq. (1.1) hold, for all $t \in \mathbb{R}$,

$$
\begin{gathered}
-\varepsilon<N_{\text {triv }}(t)<\varepsilon, \\
K_{\text {min }}-\varepsilon<N_{1}(t)<K_{\text {max }}+\varepsilon, \\
A_{\text {min }}-\varepsilon<N_{2}(t)<A_{\text {max }}+\varepsilon .
\end{gathered}
$$

Remark 3.3 New estimates in Corollary (3.2) are better than (3.6), but are not sharp. Our technique requires control of the sign of the right-hand side of Eq. (1.1) and, unfortunately, does not allow further tightening of inequalities (3.7).

\subsubsection{Existence of Periodic Solutions and their Stability for $A(t)=0$}

In this subsection, we investigate the existence of the periodic solutions to Eq. (1.1) and their stability when the Allee threshold $A(t)$ is zero (weak Allee effect).

Consider the case when $A(t)=0$. Then, Eq. (1.1) takes the form

$$
\dot{N}(t)=\frac{r(t)}{K(t)} N^{2}(t)\left(1-\frac{N(t)}{K(t)}\right)
$$

whereas assumption (3.1) is satisfied automatically. 
Theorem 3.4 (Unique Positive Attractor) Eq. (3.8) has two periodic solutions, a semi-stable trivial solution $N_{\text {triv }}(t)$ and an asymptotically stable positive solution $N_{1}(t)$ that attracts all other solutions to this equation with positive initial data.

Proof. For any $M^{*}>K_{\max }$, one has, for all $t \geq t_{0}$,

$$
\begin{aligned}
f\left(t, M^{*}\right) & =\frac{r(t)}{K^{2}(t)}\left(M^{*}\right)^{2}\left(K(t)-M^{*}\right) \\
& \leq \frac{r_{\min }}{K_{\max }^{2}}\left(M^{*}\right)^{2}\left(K_{\max }-M^{*}\right)<0
\end{aligned}
$$

and

$$
\begin{aligned}
f\left(t, \frac{K_{\min }}{2}\right) & =\frac{r(t)}{K^{2}(t)}\left(\frac{K_{\min }}{2}\right)^{2}\left(K(t)-\frac{K_{\min }}{2}\right) \\
& \geq \frac{r_{\min } K_{\min }^{3}}{8 K_{\max }^{2}}>0 .
\end{aligned}
$$

Thus, horizontal lines $\beta_{1}(t)=M^{*}$ and $\alpha_{1}(t)=K_{\min } / 2$ are strict upper and lower fences, respectively, since

$$
f\left(t, \alpha_{1}(t)\right)>\dot{\alpha}_{1}(t)=0, \quad f\left(t, \beta_{1}(t)\right)<\dot{\beta}_{1}(t)=0 .
$$

Furthermore, $\alpha_{1}(t)<\beta_{1}(t)$, which means that these fences form a funnel; once solution enters the funnel, it stays there eventually. Therefore, Eq. (3.8) has an asymptotically stable positive periodic solution $N_{1}(t)$ satisfying

$$
\frac{K_{\min }}{2}<N_{1}(t)<M^{*} .
$$

On the other hand, 


$$
\begin{aligned}
f\left(t,-\frac{K_{\min }}{2}\right) & =\frac{r(t)}{K^{2}(t)}\left(-\frac{K_{\min }}{2}\right)^{2}\left(K(t)+\frac{K_{\min }}{2}\right) \\
& \geq \frac{r_{\text {min }}}{K_{\max }^{2}}\left(-\frac{K_{\min }}{2}\right)^{2}\left(K_{\text {min }}+\frac{K_{\min }}{2}\right) \\
& =\frac{3 r_{\min } K_{\min }^{3}}{8 K_{\max }^{2}}>0 .
\end{aligned}
$$

This means that the trivial solution $N_{\text {triv }}(t)$ is a semi-stable periodic solution to Eq. (3.8); it attracts solutions with negative initial data and repels those with positive initial data; there are no more periodic solutions in this case.

Remark 3.5 Observe that in the case $A(t)=0$, solutions to Eq. (3.8) with positive initial data exhibit logistic type dynamics similar to that observed for the logistic differential equation

$$
\dot{N}(t)=r(t) N(t)\left(1-\frac{N(t)}{K(t)}\right)
$$

although solutions to Eq. (3.8) do not approach a unique positive solution $N_{1}(t)$ as fast as the solution of Eq. (3.9).

The following proposition is similar to Corollary (3.2).

Corollary 3.6 Let $A(t)=0$. Then, for any $\varepsilon>0$ and for all $t \in \mathbb{R}$, the first two estimates in (3.7) hold for the periodic solutions $N_{\text {triv }}(t)$ and $N_{1}(t)$ to Eq. (1.1).

\subsubsection{Existence of Periodic Solutions and their Stability for $A(t)<0$}

In this subsection, we examine the existence of the periodic solution and their stability for the case $A(t)<0$ (weak Allee effect). 
Theorem 3.7 (Positive and Negative Attractors) Let $A(t)<0$ and assume that

$$
0<-A_{\min }<K_{\min }
$$

Then Eq. (1.1) has three periodic solutions: the trivial solution $N_{\text {triv }}(t)$ which is unstable, a positive solution $N_{1}(t)$ and a negative solution $N_{2}(t)$, both asymptotically stable. Furthermore, $N_{1}(t)$ attracts all solutions with positive initial values.

Proof. Pick an $M^{*}>K_{\max }$, then

$$
\begin{aligned}
f\left(t, M^{*}\right) & =\frac{r(t)}{K^{2}(t)} M^{*}\left(K(t)-M^{*}\right)\left(M^{*}-A(t)\right) \\
& \leq \frac{r_{\min }}{K_{\max }^{2}} M^{*}\left(K_{\max }-M^{*}\right)\left(M^{*}-A_{\max }\right)<0 .
\end{aligned}
$$

Noting that, by virtue of (3.10), $A_{\max }+K_{\min }>0$, we conclude that

$$
\begin{aligned}
& f\left(t, \frac{A_{\max }+K_{\min }}{2}\right)=\frac{r(t)}{K^{2}(t)}\left(\frac{A_{\max }+K_{\min }}{2}\right)\left(K(t)-\frac{A_{\max }+K_{\min }}{2}\right) \\
& \times\left(\frac{A_{\text {max }}+K_{\text {min }}}{2}-A(t)\right) \geq \frac{r_{\text {min }}}{K_{\text {max }}^{2}}\left(\frac{A_{\text {max }}+K_{\text {min }}}{2}\right) \\
& \times\left(K_{\min }-\frac{A_{\max }+K_{\min }}{2}\right)\left(\frac{A_{\max }+K_{\min }}{2}-A_{\max }\right) \\
& =\frac{r_{\min }}{8 K_{\max }^{2}}\left(A_{\max }+K_{\min }\right)\left(K_{\min }-A_{\max }\right)^{2}>0 .
\end{aligned}
$$

Therefore, $\beta_{1}(t)=M^{*}$ and $\alpha_{1}(t)=\left(A_{\max }+K_{\min }\right) / 2$ are, respectively, strict upper and lower fences since

$$
f\left(t, \alpha_{1}(t)\right)>\dot{\alpha}_{1}(t)=0, \quad f\left(t, \beta_{1}(t)\right)<\dot{\beta}_{1}(t)=0 .
$$

Furthermore, $\alpha_{1}(t)<\beta_{1}(t)$, and the two horizontal lines, $\beta_{1}(t)=M^{*}$ and $\alpha_{1}(t)=\left(A_{\max }+K_{\min }\right) / 2$ form a funnel, which means that there exists an 
asymptotically stable as $t \rightarrow \infty$ positive periodic solution $N_{1}(t)$ to Eq. (1.1) satisfying

$$
\frac{A_{\max }+K_{\min }}{2}<N_{1}(t)<M^{*}
$$

for all $t \in \mathbb{R}$. All other solutions with positive initial data enter the funnel and stay there eventually.

Observe also that, by virtue of Lemma (2.3.9),

$$
\begin{aligned}
f\left(t, \frac{A_{\max }}{2}\right) & =\frac{r(t)}{K^{2}(t)}\left(\frac{A_{\max }}{2}\right)\left(K(t)-\frac{A_{\max }}{2}\right)\left(\frac{A_{\max }}{2}-A(t)\right) \\
& \leq \frac{r_{\min }}{K_{\max }^{2}}\left(\frac{A_{\max }}{2}\right)\left(K_{\min }-\frac{A_{\max }}{2}\right)\left(\frac{A_{\max }}{2}-A_{\max }\right) \\
& =-\frac{r_{\min } A_{\max }^{2}}{8 K_{\max }^{2}}\left(2 K_{\min }-A_{\max }\right)<0
\end{aligned}
$$

and, for any $h^{*}<A_{\min }<0$,

$$
\begin{aligned}
f\left(t, h^{*}\right) & =\frac{r(t)}{K^{2}(t)} h^{*}\left(K(t)-h^{*}\right)\left(h^{*}-A(t)\right) \\
& \geq \frac{r_{\text {min }}}{K_{\max }^{2}} h^{*}\left(K_{\min }-h^{*}\right)\left(h^{*}-A_{\min }\right)>0 .
\end{aligned}
$$

Therefore, $\beta_{0}(t)=A_{\max } / 2$ and $\alpha_{0}(t)=\left(A_{\max }+K_{\min }\right) / 2$ are strict upper and lower fences, respectively, because

$$
\dot{\alpha}_{0}(t)=0<f\left(t, \alpha_{0}(t)\right), \quad \dot{\beta}_{0}(t)=0>f\left(t, \beta_{0}(t)\right) .
$$

They form an antifunnel since $\alpha_{0}(t)>\beta_{0}(t)$. The trivial solution $N_{\text {triv }}(t)$ to Eq. (1.1), contained in the antifunnel, is unstable and repels other solutions with close initial data. On the other hand, we observe that the second nontrivial periodic solution $N_{2}(t)$ takes on only negative values and attracts all nearby solutions. It is 
not difficult to see that $N_{2}(t)$ is located in a funnel formed by a strict upper solution $\beta_{2}(t)=A_{\max } / 2$ and a strict lower solution $\alpha_{2}(t)=h^{*}$; solutions with close initial data are eventually trapped inside the funnel.

\subsection{Blow Up Time}

In this section, the solutions to Eq. (1.1) may not be defined for all values of $t$; some may blow up in finite time forward or backward, which prompts possibility for existence of vertical asymptotes for solutions. Direct integration of Eq. (1.1) is not possible despite of its relatively simple structure. Therefore, to estimate the behavior of solutions we use upper and lower solutions to associated differential equations with constant coefficients whose exact solutions can be easily obtained in a closed form. In the sequel, we explore behavior of solutions to Eq. (1.1) satisfying the initial condition

$$
N\left(t_{0}\right)=N_{0}
$$

The following result plays the key role in our subsequent discussion.

Lemma 3.8 (Comparison Lemma) Assume that $A(t)>0$ and (3.1) are satisfied.

Then, for $N_{0}>K_{\max }$, every solution $N(t)$ to Eq. (1.1) satisfies, for all $t \geq t_{0}$,

$$
\eta(t) \leq N(t) \leq \varphi(t)
$$

where $\eta$ and $\varphi$ are solutions to differential equations

$$
\dot{\eta}(t)=-\frac{r_{\max }}{K_{\min }^{2}} \eta^{3}(t)
$$


and

$$
\dot{\varphi}(t)=\frac{r_{\min }}{K_{\max }^{2}}\left(K_{\max }-\varphi(t)\right)^{3}
$$

with the same initial data. For $t<t_{0}$, the functions $\eta$ and $\varphi$ in (3.12) swap the roles. Similarly, for $N_{0}<0$, every solution $N(t)$ to Eq. (1.1) satisfies, for all $t \geq t_{0}$,

$$
\phi(t) \leq N(t) \leq \psi(t)
$$

where $\phi$ and $\psi$ are solutions to differential equations

$$
\dot{\phi}(t)=-\frac{r_{\min }}{K_{\max }^{2}} \phi^{3}(t)
$$

and

$$
\dot{\psi}(t)=\frac{r_{\max }}{K_{\min }^{2}}\left(K_{\max }-\psi(t)\right)^{3}
$$

satisfying the same initial condition. For $t<t_{0}$, the functions $\phi$ and $\psi$ in (3.15) swap the roles.

Proof. For $N_{0}>K_{\max }$, one has, for all $t \geq t_{0}$,

$$
\begin{aligned}
N(t) & =\frac{r(t)}{K^{2}(t)} N(t)(K(t)-N(t))(N(t)-A(t)) \\
& \leq \frac{r_{\min }}{K_{\max }^{2}} N(t)\left(K_{\max }-N(t)\right)\left(N(t)-A_{\max }\right) \\
& \leq \frac{r_{\min }}{K_{\max }^{2}}\left(K_{\max }-N(t)\right)^{3}
\end{aligned}
$$

and 


$$
\begin{aligned}
\dot{N}(t) & =\frac{r(t)}{K^{2}(t)} N(t)(K(t)-N(t))(N(t)-A(t)) \\
& \geq \frac{r_{\text {max }}}{K_{\min }^{2}} N(t)\left(K_{\min }-N(t)\right)\left(N(t)-A_{\min }\right) \\
& \geq-\frac{r_{\max }}{K_{\min }^{2}} N(t)^{3} .
\end{aligned}
$$

In a similar manner, for $N_{0}<0$, we obtain the following estimates:

$$
\begin{aligned}
N(t) & =\frac{r(t)}{K^{2}(t)} N(t)(K(t)-N(t))(N(t)-A(t)) \\
& \leq \frac{r_{\max }}{K_{\min }^{2}} N(t)\left(N(t)-A_{\max }\right)\left(K_{\max }-N\right) \\
& \leq \frac{r_{\max }}{K_{\min }^{2}}\left(K_{\max }-N(t)\right)^{3}
\end{aligned}
$$

and

$$
\begin{aligned}
N(t) & =\frac{r(t)}{K^{2}(t)} N(t)(K(t)-N(t))(N(t)-A(t)) \\
& \geq-\frac{r_{\min }}{K_{\max }^{2}} N(t)\left(K_{\min }-N(t)\right)\left(N(t)-A_{\min }\right) \\
& \geq-\frac{r_{\min }}{K_{\max }^{2}} N^{3}(t),
\end{aligned}
$$

for all $t \geq t_{0}$. The proof is complete.

Corollary 3.9 (i) Let $A(t)=0$. Then both conclusions of Lemma (3.8) regarding solutions with large positive and negative initial data remain intact.

(ii) Let $A(t)<0$ and assume that (3.10) holds. Then, the first conclusion of Lemma (3.8) for solutions with large positive data remains intact. The second conclusion holds for solutions satisfying condition

$$
N_{0}<A_{\min }<0
$$


Proof. We note that the only change in the proof regards the second conclusion in the case (ii). We have to require that (3.18) holds to ensure that the term $N(t)-A(t)$ on the right hand side is always negative. The rest of the proof is as in Lemma (3.8). Using Lemma (3.8), one can describe more precisely backward behavior of solutions to Eq. (1.1) satisfying the initial condition (3.11).

Theorem 3.10 (i) Suppose that $A(t)>0$ and (3.1) is satisfied. Then backward blow up time $t_{\text {bul }}$ for solutions to Eq. (1.1) with large initial data $N_{0}>K_{\max }$ satisfies the estimates

$$
t_{\varphi}^{*}=t_{0}-\frac{K_{\max }^{2}}{2 r_{\min }\left(K_{\max }-N_{0}\right)^{2}} \leq t_{\mathrm{bul}} \leq t_{0}-\frac{K_{\min }^{2}}{2 r_{\max } N_{0}^{2}}=t_{\eta}^{*} .
$$

For solutions with negative initial data, the estimates for the backward blow up time $t_{\text {bun }}$ take the form

$$
t_{\phi}^{*}=t_{0}-\frac{K_{\max }^{2}}{2 r_{\min } N_{0}^{2}} \leq t_{\text {bun }} \leq t_{0}-\frac{K_{\min }^{2}}{2 r_{\max }\left(K_{\max }-N_{0}\right)^{2}}=t_{\psi}^{*}
$$

(ii) Let $A(t)=0$. Then conclusions in (i) remain intact.

(iii) Suppose that $A(t)<0$ and (3.10) holds. Then (3.19) is satisfied for solutions with large positive data, whereas (3.20) holds for solutions satisfying (3.18).

Proof. (i) Let $N(t)$ be the solution to Eq. (1.1) satisfying the initial condition (3.11). Then, by virtue of Lemma (3.8), for all $t \in \mathbb{R}, N(t)$ is squeezed (note the order swapping in the inequalities at $t=t_{0}$ ) between solutions to differential equations 
(3.13) and (3.14) starting at $\left(t_{0}, N_{0}\right)$. A straightforward integration of Eq. (3.14) from $t_{0}$ to $t$ yields

$$
\frac{1}{\left(\varphi(t)-K_{\max }\right)^{2}}=\frac{1}{\left(\varphi\left(t_{0}\right)-K_{\max }\right)^{2}}+2 \frac{r_{\min }}{K_{\max }^{2}}\left(t-t_{0}\right)
$$

or, equivalently,

$$
\varphi(t)=\frac{K_{\max } \sqrt{\left(\varphi\left(t_{0}\right)-K_{\max }\right)^{-2}+2 r_{\min } K_{\max }^{-2}\left(t-t_{0}\right)}+1}{\sqrt{\left(\varphi\left(t_{0}\right)-K_{\max }\right)^{-2}+2 r_{\min } K_{\max }^{-2}\left(t-t_{0}\right)}} .
$$

Therefore, solution $\varphi(t)$ to Eq. (3.14) satisfying (3.11) blows up backward in time at the instant

$$
t_{\varphi}^{*}=t_{0}-\frac{K_{\max }^{2}}{2 r_{\min }\left(K_{\max }-\varphi\left(t_{0}\right)\right)^{2}}
$$

this solution has a vertical asymptote $t=t_{\varphi}^{*}$. Similarly, integrating Eq. (3.13) between $t_{0}$ to $t$, one has

$$
\frac{1}{\eta^{2}(t)}=\frac{1}{\eta^{2}\left(t_{0}\right)}+2 \frac{r_{\max }}{K_{\min }^{2}}\left(t-t_{0}\right)
$$

or

$$
\eta(t)=\frac{1}{\sqrt{\eta^{-2}\left(t_{0}\right)+2 r_{\max } K_{\min }^{-2}\left(t-t_{0}\right)}} .
$$

Consequently, solution $\eta(t)$ to Eq. (3.13) satisfying (3.11) blows up backward in time at the instant

$$
t_{\eta}^{*}=t_{0}-\frac{K_{\min }^{2}}{2 r_{\max } \eta^{2}\left(t_{0}\right)}
$$


and has a vertical asymptote $t=t_{\eta}^{*}$. The estimate (3.19) accounts for the change of order in (3.12). Following the same lines, one can also evaluate backward blow up time for solutions to Eq. (1.1) with negative initial values. Using equations (3.16) and (3.17) rather than (3.13) and (3.14), after a straightforward integration from $t_{0}$ to $t$, one deduces explicit formulas for solutions of the second pair of equations:

$$
\phi(t)=\frac{1}{\sqrt{\phi^{-2}\left(t_{0}\right)+2 r_{\min } K_{\max }^{-2}\left(t-t_{0}\right)}}
$$

and

$$
\psi(t)=\frac{K_{\max } \sqrt{\left(K_{\max }-\psi\left(t_{0}\right)\right)^{-2}+2 r_{\min } K_{\max }^{-2}\left(t-t_{0}\right)}+1}{\sqrt{\left(K_{\max }-\psi\left(t_{0}\right)\right)^{-2}+2 r_{\min } K_{\max }^{-2}\left(t-t_{0}\right)}} .
$$

Corresponding blow up times are

$$
t_{\phi}^{*}=t_{0}-\frac{K_{\max }^{2}}{2 r_{\min } \phi^{2}\left(t_{0}\right)}
$$

and

$$
t_{\psi}^{*}=t_{0}-\frac{K_{\min }^{2}}{2 r_{\max }\left(K_{\max }-\psi\left(t_{0}\right)\right)^{2}} .
$$

Taking into account that $\phi$ and $\psi$ swap in (3.15) for $t<t_{0}$, these two equations lead to the estimate (3.20) for the backward blow up time for solutions to Eq. (1.1) with negative initial data. The proof in the case (ii) is the same, whereas in the case (iii) only obvious minor modifications are required.

Remark 3.11 The estimates provided by solutions to differential equations (3.13)(3.14) are not very tight as time $t$ advances. This can be seen from the figures in 
the next chapter. The reason is that all differential equations are in the one and only form that allows to determine explicit solutions. However, the estimates for the blow up time are reasonably good, especially those provided by solutions to Eq. (3.13) for large positive initial values and by solutions to Eq. (3.14) for negative initial values.

\subsection{Example and Discussion}

In this section, we discuss examples that illustrate results of Section 3.1 and Section 3.2. To underline the changes in the dynamics of the population that occur during the transition of the Allee threshold $A(t)$ from positive values through zero to negative ones, we intentionally keep the other two parameters of the system, the intrinsic growth rate $r(t)$ and carrying capacity $K(t)$, unchanged. We also compare our technique to the approach suggested in the recent work by Padhi [14].

Example 3.12 For $\gamma \in \mathbb{R}$, consider Eq. (1.1) with

$$
r(t)=\sin 2 \pi t+4, \quad K(t)=\cos 2 \pi t+8, \quad A(t)=\gamma(\sin 2 \pi t+2)
$$

This choice of coefficients leads to the differential equation

$$
\dot{N}(t)=(\sin 2 \pi t+4) N(t)\left(1-\frac{N(t)}{\cos 2 \pi t+8}\right) \times\left(\frac{N(t)}{\cos 2 \pi t+8}-\gamma \frac{\sin 2 \pi t+2}{\cos 2 \pi t+8}\right)
$$

To begin with, let $\gamma=1$. Obviously, then $A(t)>0$ and $A_{\max }=3 \leq 7=K_{\min }$. By Theorem (3.1), Eq. (3.21) has three periodic solutions: an asymptotically stable trivial solution $N_{\text {triv }}(t)=0$ and two positive solutions, an asymptotically stable solution $N_{1}(t)$ and an unstable solution $N_{2}(t)$.

By Corollary (3.2), these three solutions satisfy the inequalities 


$$
\begin{gathered}
-\varepsilon<N_{\text {triv }}(t)<\varepsilon, \\
7-\varepsilon<N_{1}(t)<9+\varepsilon, \\
1-\varepsilon<N_{2}(t)<3+\varepsilon,
\end{gathered}
$$

for any $\varepsilon>0$, see Figure 1 .

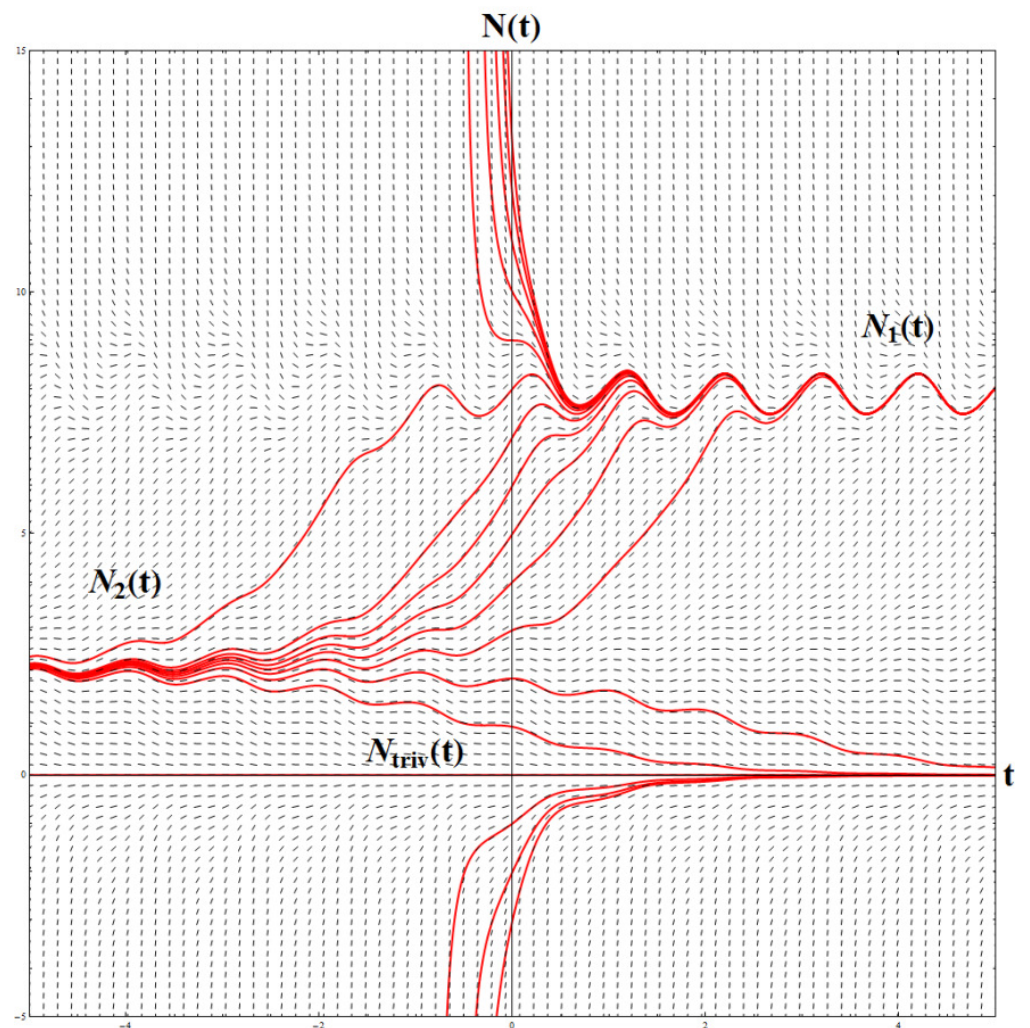

Figure 1: Three periodic solutions and several other solutions to Eq. (3.21) for $\gamma=1$.

This and all other figures in this chapter have been plotted with the help of the computer algebra system Mathematica. Differential equations (3.13)-(3.17) that define upper and lower solutions for Eq. (3.21) have the form 


$$
\begin{aligned}
& \dot{\eta}(t)=-\frac{5}{49} \eta^{3}(t), \\
& \dot{\varphi}(t)=\frac{1}{27}(9-\phi(t))^{3}, \\
& \dot{\phi}(t)=-\frac{1}{27} \varphi^{3}(t), \\
& \dot{\psi}(t)=\frac{5}{49}(9-\psi(t))^{3} .
\end{aligned}
$$

The estimates (3.19) and (3.20) for the backward blow up times provided in Theorem (3.10) yield for Eq. (3.21) the following. For solutions with large initial data,

$$
t_{0}-\frac{27}{2\left(9-N_{0}\right)^{2}} \leq t_{\text {bul }} \leq t_{0}-\frac{49}{10 N_{0}^{2}}
$$

whereas for solutions with negative initial data, the estimates take the form

$$
t_{0}-\frac{27}{2 N_{0}^{2}} \leq t_{\text {bun }} \leq t_{0}-\frac{49}{10\left(9-N_{0}\right)^{2}}
$$

Periodic solutions to Eq. (3.21) are shown in Figure 2 along with several solutions approaching asymptotically stable periodic solution $N_{1}(t)$.

For solutions departing from the points $(0,13)$ and $(0,-3)$, the estimates for the backward blow up time are, respectively,

$$
-\frac{27}{32} \leq t_{\text {bul }} \leq-\frac{49}{1690}, \quad-\frac{27}{338} \leq t_{\text {bun }} \leq-\frac{49}{1600}
$$

and 


$$
-\frac{27}{288} \leq t_{\text {bul }} \leq-\frac{49}{90}, \quad-\frac{3}{2} \leq t_{\text {bun }} \leq-\frac{49}{1440}
$$

Two pairs of vertical asymptotes are shown, along with several trajectories, in Figure 2. Taking into account that the difference $t_{\eta}^{*}-t_{\psi}^{*}$ is usually very small, it is quite difficult to distinguish asymptotes to solutions to Eqs. (3.13) and (3.17) plotted together. In fact, for both pairs of asymptotes to solutions through $(0,13)$ and $(0,-3)$, one has

$$
t_{\eta}^{*}-t_{\psi}^{*}=\frac{49}{1600}-\frac{49}{1690}=49\left(\frac{1}{1600}-\frac{1}{1690}\right)=1.6309 \times 10^{-3}
$$

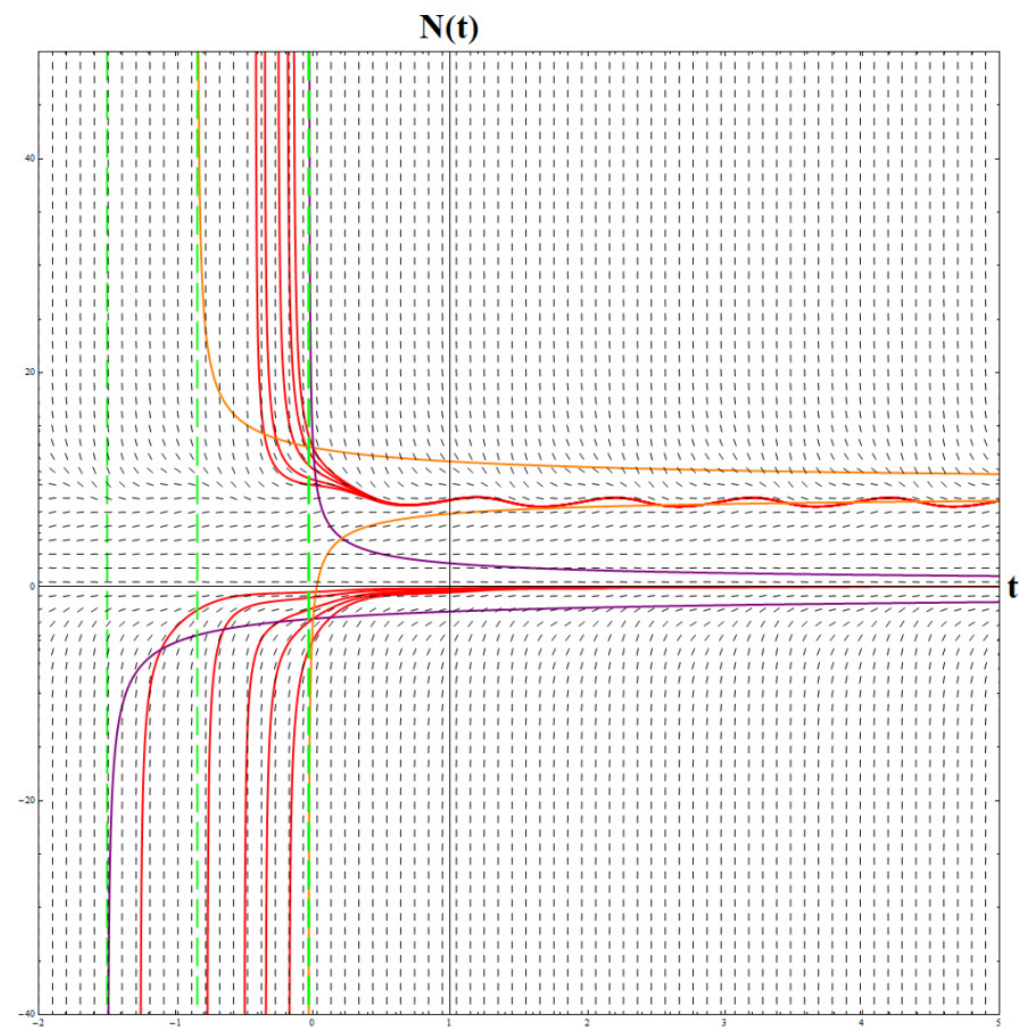

Figure 2: Vertical asymptotes (green), upper (orange) and lower (lilac) solutions along with several other solutions (red) to Eq. (3.21) for $\gamma=1$.

This is why one can see only three asymptotes in Figure 2, the upmost right being slightly thicker and representing two asymptotes that collide rather than one. Note that, by virtue of Lemma (3.8), vertical asymptotes do not depend on the choice of 
$A(t)$. This is a natural consequence of the restrictions (3.1) and (3.10) imposed on the coefficient associated with the Allee effect $A(t)$ simply leaves no trace in Eqs. (3.13)-(3.17). Consequently, variations in $A(t)$ in Eq. (3.21) do not affect their location.

In order to compare our results with those derived by Padhi [14], observe that, by virtue of (1.5), one obtains for Eq. (3.21) the following values:

$$
M=\int_{0}^{1}(\sin (2 \pi t)+4) d t=4
$$

and

$$
L=\int_{0}^{1} \frac{(\sin (2 \pi t)+4)(\sin (2 \pi t)+2)}{8+\cos (2 \pi t)} d t=-\frac{55}{21} \sqrt{7}+8 \approx 1.0707
$$

A straightforward computation yields

$$
\begin{aligned}
& \alpha=\frac{1}{2 \cdot 4} \times\left(\sqrt{\left(4-\frac{55}{21} \sqrt{7}+8\right)^{2}-4 \cdot 4 \exp \left(\frac{55}{21} \sqrt{7}-8\right)\left(\exp \left(-\frac{55}{21} \sqrt{7}+8\right)-1\right)}\right. \\
& \left.+4-\frac{55}{21} \sqrt{7}+8\right) \approx 1.1211
\end{aligned}
$$

and

$$
\beta=\frac{\exp \left(2 \cdot\left(-\frac{55}{21} \sqrt{7}+8\right)\right)-\exp \left(\frac{55}{21} \sqrt{7}-8\right)}{4-\frac{55}{21} \sqrt{7}+8} \approx 1.6108
$$

Therefore, condition (1.6) in Theorem (1.1) fails to hold for Eq. (3.21) because $\alpha<\beta$. Thus, the cited theorem does not apply to this equation. Serious limitations brought by applying a much more general result derived by Padhi [14] for Eq. (1.3) 
to its particular case, Eq. (1.4) are clearly seen, whereas our simpler technique tailored for the latter equation proves to be more efficient. In fact, Theorem (1.1) does not bring much information to mathematical biologists who require more details regarding the exact number of positive periodic solutions and their qualitative properties.

Letting now $\gamma=0$ in Eq. (3.21), we obtain

$$
\dot{N}(t)=\frac{\sin 2 \pi t+4}{\cos 2 \pi t+8} N^{2}(t)\left(1-\frac{N(t)}{\cos 2 \pi t+8}\right) .
$$

By Theorem (3.4), Eq. (3.25) has two periodic solutions, a semi-stable trivial solution $N_{\text {triv }}(t)$ and an asymptotically stable positive solution $N_{1}(t)$ that attracts all solutions to this equation with positive initial data. Both solutions are sketched in Figure 3. The first two estimates in (3.22), as well as the estimates (3.23) and (3.24) for the backward blow up times remain valid also for solutions to Eq. (3.25) with large and negative initial data respectively. 


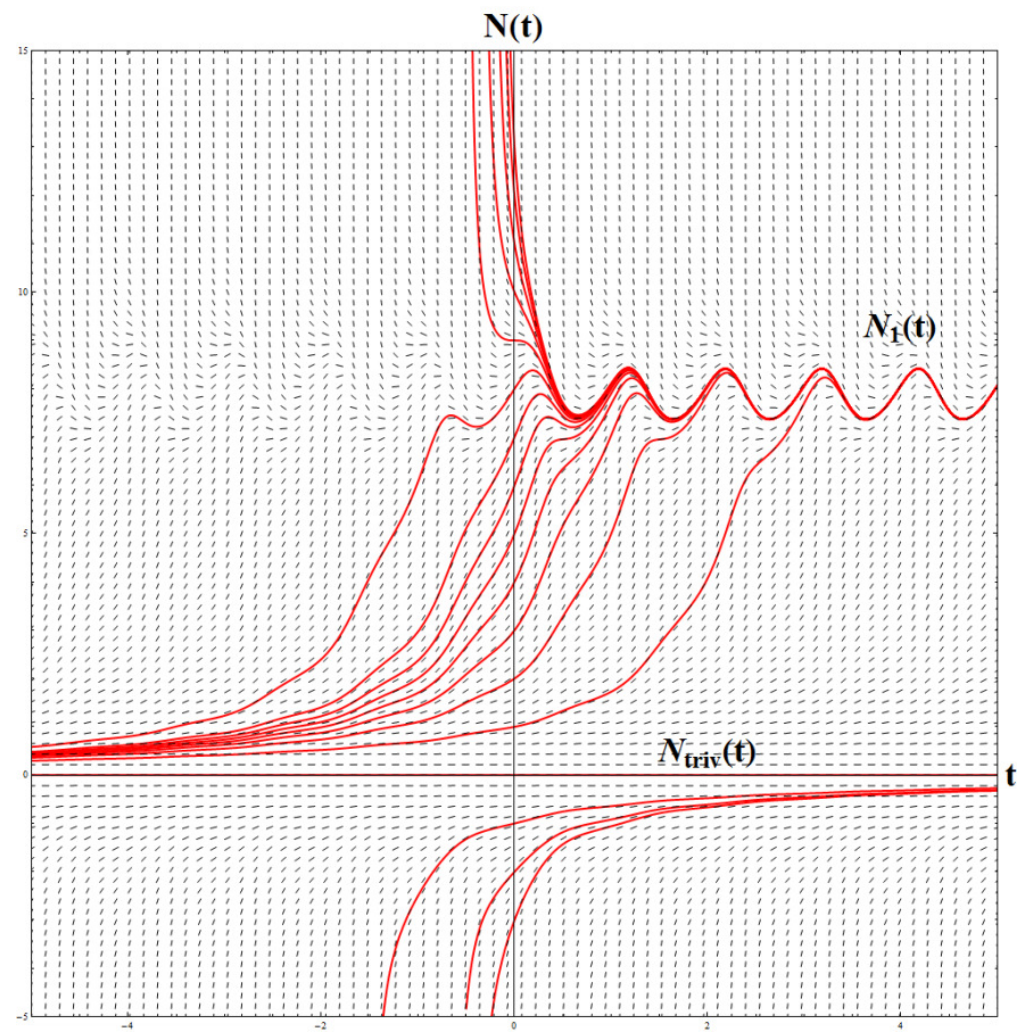

Figure 3: Two periodic solutions and several other solutions to Eq. (3.21) for $\gamma=0$.

Finally, choosing $\gamma=-1$ in Eq. (3.21), one arrives at the differential equation

$$
\dot{N}(t)=(\sin 2 \pi t+4) N(t)\left(1-\frac{N(t)}{\cos 2 \pi t+8}\right)\left(\frac{N(t)+\sin 2 \pi t+2}{\cos 2 \pi t+8}\right) .
$$

Observe that (3.10) is satisfied since $A_{\min }=-3, \quad K_{\min }=7$, and $K_{\min }+A_{\min }=4>0$. Consequently, by Theorem (3.7), Eq. (3.26) has three periodic solutions, an unstable trivial solution $N_{\text {triv }}(t)$, an asymptotically stable positive solution $N_{1}(t)$ and an asymptotically stable negative solution $N_{2}(t)$, see Figure 4. 


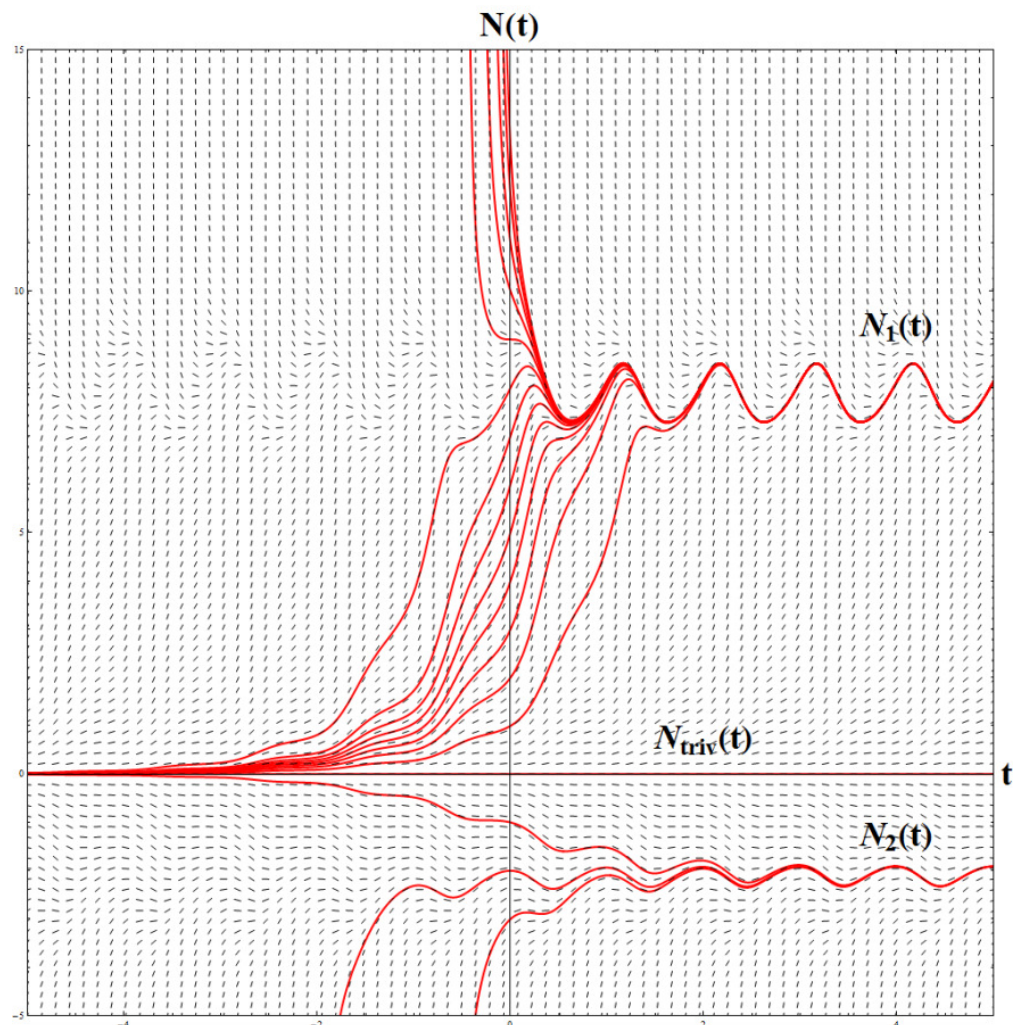

Figure 4: Three periodic solutions and several other solutions to Eq. (3.21) for $\gamma=-1$.

Note that $N_{1}(t)$ attracts all solutions with positive initial values. As above, the estimates (3.23) and (3.24) for the backward blow up times also apply to solutions to Eq. (3.26) with large positive initial data and solutions with negative initial data satisfying (3.18).

We would like to stress that despite of its generality and power, the method using an advanced multiple fixed point theorem to Eqs. (1.3) and (1.4) suggested by Padhi [14] does not apply to the latter equation whenever condition (1.6) fails to hold; this fact was observed for Eq. (3.21) for $\gamma=1$; more equations with similar properties can be easily provided. Furthermore, the example selected by Padhi [14] to illustrate Theorem (1.1) looks quite artificial and may not be suitable for describing the dynamics of the real system due to rather unrealistic ranges of the carrying capacity $c(t)=(1.2+\sin t)^{-1}$ and intrinsic growth rate $a(t)=(1.2+\sin t)^{2}$. 
In fact; the maximal value $a_{\max }=121 / 25$ is very close to the maximum of the carrying capacity $c_{\max }=5$,

$$
\frac{5}{11} \leq c(t) \leq 5 \quad \text { and } \quad \frac{1}{25} \leq a(t) \leq \frac{121}{25}
$$

Finally, we note that Padhi [14] do not discuss weak Allee effect.

In this light, our approach combining rigorous mathematical analysis with direction field arguments and the upper and lower solutions method is more efficient for Eq. (1.1); it provides all necessary information on the dynamics of the species under nonrestrictive and easily verifiable assumptions like (3.1), (3.10) or (3.18). Such simple but efficient approach is very important in applied problems arising in population dynamics or epidemiology. One should note that since the suggested technique relies on the form of the right-hand side in a given differential equation, its efficiency is directly related to estimates for the right-hand side used in Section 3.1 or estimates for solutions derived in Section 3.2 and thus differs for various classes of differential equations.

However, similar ideas proved to be useful for the study of several population models, see, for instance, the direction field arguments in the analysis of periodic logistic and Gompertz equations with harvesting in Brauer and Sánchez [4] and applications of the comparison technique to periodic competing species in [30] or [31], as well as the references cited therein. Very recently, Hasanbulli [32] used the technique described in this thesis for the study of the effect of a periodic constant yield harvesting on a single species population whose dynamics in a fluctuating environment is described by the logistic differential equation with periodic coefficients. They demonstrated the existence of the attractor-repellor pair, obtained 
efficient bounds for positive attractor and repellor along with the estimates for extinction time and for forward/backward blow up times.

In what concerns dynamics of the general differential equation (1.3), we would like to mention a very interesting contribution by Bardi [33] for a general nonlinear differential equation

$$
\frac{d x(t)}{d t}=x(t)(r(t)-g(t, x(t)))
$$

where $r$ is continuous $T$ - periodic function, $g: \mathbb{R} \times[0,+\infty) \rightarrow \mathbb{R}$ is continuous $T$ - periodic in $t$ and continuously differentiable in $x$ function such that $g(t, 0) \equiv 0$. In the cited paper, existence, multiplicity and global stability results for Eq. (3.27) have been proved. We believe that an application of Bardi's approach based on the analysis of the Poincaré map associated to Eq. (3.27) to the problem studied by Padhi [14] for Eq. (1.3) would be much more beneficial. 


\section{Chapter 4}

\section{EXISTENCE OF PERIODIC SOLUTIONS OF THE DIFFERENTIAL EQUATION WITH ALLEE EFFECT UNDER HARVESTING}

In this chapter, we study the existence of periodic solutions to Eq. (1.7) and their stability properties. In the following theorems, we show the corridors where the periodic solutions located. Our technique requires control of the sign of the righthand side of Eq. (1.7). Eight cases where the Allee threshold $A(t)$ is positive, zero, negative and the harvesting $h(t)$ is positive, negative considered separately.

Let the minimal and maximal values for the growth rate, carrying capacity, Allee threshold and harvesting effort,

$$
\begin{aligned}
& r_{\max }=\max _{t \in[0,+\infty)} r(t), r_{\min }=\min _{t \in[0,+\infty)} r(t), K_{\max }=\max _{t \in[0,+\infty)} K(t), K_{\min }=\min _{t \in[0,+\infty)} K(t) \\
& A_{\max }=\max _{t \in[0,+\infty)} A(t), A_{\min }=\min _{t \in[0,+\infty)} A(t), h_{\max }=\max _{t \in[0,+\infty)} h(t), h_{\min }=\min _{t \in[0,+\infty)} h(t) .
\end{aligned}
$$

If $A(t)>0$ then

$$
A_{\min } \leq A_{\max }<K_{\min } \leq K_{\max }
$$

If $A(t)<0$ then

$$
\left|A_{\max }\right| \leq\left|A_{\min }\right|<K_{\min } \leq K_{\max }
$$


Consider the square on the right-hand side of Eq. (1.7) in the form

$$
\begin{aligned}
\dot{N}(t)= & -\frac{r(t)}{K^{2}(t)} N(t)\left(N(t)-\left(\frac{K(t)+A(t)}{2}\right)\right)^{2} \\
& -\frac{r(t)}{K^{2}(t)} N(t)\left(\frac{K^{2}(t)}{r(t)} h(t)-\left(\frac{K(t)-A(t)}{2}\right)^{2}\right) .
\end{aligned}
$$

The derivative $\frac{d N}{d t}=\dot{N}(t)$ is negative for all $t \in \mathbb{R}$, we provided that the harvesting $h(t)$ satisfied the condition

$$
\left(\frac{K^{2}(t)}{r(t)} h(t)-\left(\frac{K(t)-A(t)}{2}\right)^{2}\right)>0
$$

in which case the population density $N(t) \rightarrow 0$ as $t \rightarrow+\infty$, the Eq. (1.7) has zero solution and the population goes extinct. Supposing that the opposite to inequality (4.3) holds for all $t \in \mathbb{R}$, we obtain the existence of periodic solutions. Since $h(t)<0$, $D=(K(t)+A(t))^{2}-4\left(K(t) A(t)+\frac{K^{2}(t)}{r(t)} h(t)\right)=4\left(\left(\frac{K(t)-A(t)}{2}\right)^{2}-\frac{K^{2}(t)}{r(t)} h(t)\right)>0$.

Then, the solutions of the equation $f(N, t)=0$ are

$$
N_{1}(t)=\frac{(K(t)+A(t))+\sqrt{(K(t)+A(t))^{2}-4\left(K(t) A(t)+\frac{K^{2}(t)}{r(t)} h(t)\right)}}{2}>0
$$

and 


$$
N_{2}(t)=\frac{(K(t)+A(t))-\sqrt{(K(t)+A(t))^{2}-4\left(K(t) A(t)+\frac{K^{2}(t)}{r(t)} h(t)\right)}}{2}
$$

One of the solutions is positive; the other one is positive or negative, depending on the following.

If $A(t)+\frac{K(t)}{r(t)} h(t)>0$ provided by $h_{\min }>-\frac{A_{\min } r_{\min }}{K_{\max }}$ then, $N_{2}(t)>0$, for $t \geq 0$.

If $A(t)+\frac{K(t)}{r(t)} h(t)<0$ provided by $h_{\max }<-\frac{A_{\max } r_{\max }}{K_{\min }}$ then $N_{2}(t)<0$, for $t \geq 0$.

\subsection{Existence of Periodic Solutions}

\subsubsection{Existence of Periodic Solutions and their Stability for $A(t)>0$ and $h(t)>0$}

\section{( Harvesting for Strong Allee Effect )}

Theorem 4.1 Let $A(t)>0$ and $h(t)>0$ and harvesting satisfies the condition

$$
h_{\max }<\frac{r_{\min }}{K_{\max }^{2}}\left(\frac{K_{\min }-A_{\max }}{2}\right)^{2}
$$

then Eq. (1.7) has three periodic solutions; the asymptotically stable trivial solution $N_{\text {triv }}(t)$ and two positive solutions, asymptotically stable solution $N_{1}(t)$ and an unstable solution $N_{2}(t)$.

Proof. Let $N=K_{\max }$, then, using Lemma (2.3.9), we conclude that, for all $t \in[0,+\infty)$ 


$$
\begin{aligned}
& \dot{N}=f\left(t, K_{\text {max }}\right)=\left(K_{\text {max }}\right)\left(\frac{r(t)}{K^{2}(t)}\left(K(t)-K_{\text {max }}\right)\left(K_{\text {max }}-A(t)\right)-h(t)\right) \\
& <\left(K_{\text {max }}\right)\left(\frac{r_{\text {min }}}{K_{\text {max }}^{2}}\left(K_{\text {max }}-K_{\text {max }}\right)\left(K_{\text {max }}-A_{\text {max }}\right)-h_{\text {min }}\right) \\
& =-K_{\text {max }} h_{\text {min }}<0
\end{aligned}
$$

On the other hand,

$$
\begin{aligned}
& \dot{N}=f\left(t, \frac{A_{\text {max }}+K_{\text {min }}}{2}\right) \\
& =\left(\frac{K_{\min }+A_{\max }}{2}\right) \times\left[\frac{r(t)}{K^{2}(t)}\left(K(t)-\frac{K_{\min }+A_{\max }}{2}\right)\left(\frac{K_{\min }+A_{\max }}{2}-A(t)\right)-h(t)\right] \\
& >\left(\frac{K_{\min }+A_{\max }}{2}\right) \times\left[\frac{r_{\min }}{K_{\max }^{2}}\left(K_{\min }-\frac{K_{\min }+A_{\max }}{2}\right)\left(\frac{K_{\min }+A_{\max }}{2}-A_{\max }\right)-h_{\max }\right] \\
& =\left(\frac{K_{\min }+A_{\max }}{2}\right) \\
& \times\left[-\frac{r_{\min }}{K_{\max }^{2}}\left(\frac{K_{\min }+A_{\max }}{2}-K_{\min }\right)\left(\frac{K_{\min }+A_{\max }}{2}-A_{\max }\right)-h_{\max }\right] \\
& =\left(\frac{K_{\min }+A_{\max }}{2}\right) \\
& \times\left[-\frac{r_{\min }}{K_{\max }^{2}}\left[\left(\frac{K_{\min }+A_{\max }}{2}\right)^{2}-\left(\frac{K_{\min }+A_{\max }}{2}\right)\left(K_{\min }+A_{\max }\right)+A_{\max } K_{\min }\right]-h_{\max }\right] \\
& =\left(\frac{K_{\min }+A_{\max }}{2}\right) \times\left[-\frac{r_{\min }}{K_{\max }^{2}}\left(-\left(\frac{K_{\min }-A_{\max }}{2}\right)^{2}+A_{\max } K_{\min }\right)-h_{\max }\right] \\
& =\left(\frac{K_{\min }+A_{\max }}{2}\right)\left[\frac{r_{\min }}{K_{\max }^{2}}\left(\left(\frac{K_{\min }+A_{\max }}{2}\right)^{2}-A_{\max } K_{\min }\right)-h_{\max }\right] \\
& =\left(\frac{K_{\min }+A_{\max }}{2}\right)\left[\frac{r_{\min }}{K_{\max }^{2}}\left(\frac{K_{\min }-A_{\max }}{2}\right)^{2}-h_{\max }\right]>0
\end{aligned}
$$

Since

$$
f\left(t, \beta_{1}(t)\right)<\dot{\beta}_{1}(t)=0 \quad \text { and } \quad f\left(t, \alpha_{1}(t)\right)>\dot{\alpha}_{1}(t)=0
$$

for all $t \geq t_{0}$, we conclude that, $\beta_{1}(t)=K_{\max }$ and $\alpha_{1}(t)=\left(A_{\max }+K_{\min }\right) / 2$ are strict upper and lower fences respectively. Note that, $\alpha_{1}(t)<\beta_{1}(t)$. Therefore, there is a 
funnel that contains an asymptotically stable periodic solution $N_{1}(t)$ to Eq. (1.7). $N_{1}(t)$ attracts all other solutions with close initial data, these solutions stay in the funnel which are close in the horizontal lines $\alpha_{1}(t)$ and $\beta_{1}(t)$. The existence of a periodic solution $N_{1}(t)$ is guaranteed by Theorem (2.3.1), Corollary (2.3.2) or Theorem (2.3.4) which is corresponding theorems on funnels see Hubbard and West [18]. This solution is asymptotically stable.

Next, observe that,

$$
\begin{aligned}
\dot{N} & =f\left(t, A_{\text {min }}\right)=\left(A_{\text {min }}\right)\left(\frac{r(t)}{K^{2}(t)}\left(K(t)-A_{\text {min }}\right)\left(A_{\text {min }}-A(t)\right)-h(t)\right) \\
& <\left(A_{\text {min }}\right)\left(\frac{r_{\text {min }}}{K_{\text {max }}^{2}}\left(K_{\text {min }}-A_{\min }\right)\left(A_{\text {min }}-A_{\text {min }}\right)-h_{\text {min }}\right) \\
& =-A_{\text {min }} h_{\text {min }}<0
\end{aligned}
$$

and

$$
\begin{aligned}
\dot{N} & =f\left(t,-A_{\text {min }}\right)=\left(-A_{\min }\right)\left(\frac{r(t)}{K^{2}(t)}\left(K(t)+A_{\min }\right)\left(-A_{\text {min }}-A(t)\right)-h(t)\right) \\
& >\left(-A_{\text {min }}\right)\left(\frac{r_{\text {min }}}{K_{\text {max }}^{2}}\left(K_{\text {min }}+A_{\text {min }}\right)\left(-A_{\text {min }}-A_{\min }\right)-h_{\text {max }}\right) \\
& =\left(A_{\text {min }}\right)\left(\frac{r_{\text {min }}}{K_{\text {max }}^{2}}\left(K_{\text {min }}+A_{\text {min }}\right)\left(2 A_{\text {min }}\right)+h_{\text {max }}\right)>0
\end{aligned}
$$

Thus, the conclusion that $\beta_{0}(t)=A_{\text {min }}$ and $\alpha_{0}(t)=-A_{\text {min }}$ are strict upper and lower fences, respectively, because

$$
f\left(t, \alpha_{0}(t)\right)>\dot{\alpha}_{0}(t)=0 \text { and } \quad f\left(t, \beta_{0}(t)\right)<\dot{\beta}_{0}(t)=0
$$

and $\alpha_{0}(t)<\beta_{0}(t)$ then trivial solution $N_{\text {triv }}(t)$ to Eq. (1.7) is located in a funnel formed by the horizontal lines $\alpha_{0}(t)$ and $\beta_{0}(t)$. This solution is asymptotically stable; all solutions enter a funnel and stay there. 
Note that, by the Lemma (2.3.9), the two horizontal lines, $\beta_{2}(t)=A_{\min }$ and $\alpha_{2}(t)=\frac{A_{\max }+K_{\min }}{2}$ are strict upper and lower fences, respectively, since

$$
f\left(t, \alpha_{2}(t)\right)>\dot{\alpha}_{2}(t)=0 \text { and } \quad f\left(t, \beta_{2}(t)\right)<\dot{\beta}_{2}(t)=0 .
$$

They form an antifunnel and $\alpha_{2}(t)>\beta_{2}(t)$ as $t \rightarrow+\infty$. For an unstable periodic solution $N_{2}(t)$, as $t \rightarrow+\infty$ to Eq. (1.7) contained in the antifunnel and the other solutions with close initial value in the antifunnel. Therefore, we have established existence of three periodic solutions to Eq. (1.7), namely the trivial solution $N_{\text {triv }}(t)$ and two positive solutions, a stable solution $N_{1}(t)$ and an unstable solution $N_{2}(t)$ satisfying, for all $t \in[0,+\infty)$,

$$
-A_{\min }<N_{\text {triv }}(t)<A_{\min }<N_{2}(t)<\frac{A_{\max }+K_{\min }}{2}<N_{1}(t)<K_{\max } .
$$

By Theorem (2.3.6), Eq. (1.7) cannot have more periodic solutions. The proof is completed.

4.1.2 Existence of Periodic Solutions and their Stability for $A(t)>0$ and $h(t)<0$ ( Stocking for Weak Allee Effect )

Theorem 4.2 Let $A(t)>0$ and $h(t)<0$ and harvesting satisfies the condition,

a. $h_{\min }>-\frac{1}{4} r_{\min }\left(\frac{A_{\min }}{K_{\max }}\right)^{2}$ then Eq. (1.7) has three periodic solutions; the asymptotically stable trivial solution $N_{\text {triv }}(t)$ and two positive solutions, asymptotically stable solution $N_{1}(t)$ and an unstable solution $N_{2}(t)$ 
b. $-r_{\min }<h_{\min }<h_{\max }<-\left(r_{\max }\right)\left(\frac{A_{\max }+r_{\max }}{K_{\min }}\right)^{2}$ then Eq. (1.7) has three periodic solutions; the trivial solution $N_{\text {triv }}(t)$ which is unstable a positive solutions $N_{1}(t)$ and a negative solution $N_{2}(t)$, both asymptotically stable.

Proof.

a. For $N=A_{\min }+K_{\max }$, for all $t \geq t_{0}$,

$$
\begin{aligned}
\dot{N} & =f\left(t, A_{\text {min }}+K_{\text {max }}\right) \\
& =\left(A_{\text {min }}+K_{\text {max }}\right) \times\left(\frac{r(t)}{K^{2}(t)}\left(K(t)-\left(A_{\text {min }}+K_{\text {max }}\right)\right)\left(\left(A_{\text {min }}+K_{\text {max }}\right)-A(t)\right)-h(t)\right) \\
& <\left(A_{\text {min }}+K_{\text {max }}\right) \times\left(\frac{r_{\text {min }}}{K_{\text {max }}^{2}}\left(K_{\text {max }}-\left(A_{\text {min }}+K_{\text {max }}\right)\right)\left(\left(A_{\text {min }}+K_{\text {max }}\right)-A_{\text {max }}\right)-h_{\text {min }}\right) \\
& =\left(A_{\text {min }}+K_{\text {max }}\right)\left(-r_{\text {min }} \frac{A_{\text {min }}}{K_{\text {max }}}-h_{\text {min }}\right)<0 .
\end{aligned}
$$

Since $h_{\min }>-\frac{1}{4} r_{\min }\left(\frac{A_{\min }}{K_{\max }}\right)^{2}>-\frac{r_{\min } A_{\min }}{K_{\max }}$.

On the other hand, $\dot{N}=f\left(t, \frac{A_{\max }+K_{\min }}{2}\right)>0$, this is shown in Theorem (4.1).

There is a funnel that contains an asymptotically stable periodic solution $N_{1}(t)$ to Eq. (1.7). This solution attracts all other solutions with close initial value, these solutions stay in the funnel bounded by the horizontal lines $\beta_{1}(t)=A_{\min }+K_{\max }$, $\alpha_{1}(t)=\frac{A_{\max }+K_{\min }}{2}$ and $\alpha_{1}(t)<\beta_{1}(t)$, and the existence of the periodic solution $N_{1}(t)$ is guaranteed. Next, observe that, 


$$
\begin{aligned}
\dot{N} & =f\left(t, \frac{A_{\text {min }}}{2}\right)=\frac{A_{\text {min }}}{2}\left(\frac{r(t)}{K^{2}(t)}\left(K(t)-\frac{A_{\min }}{2}\right)\left(\frac{A_{\min }}{2}-A(t)\right)-h(t)\right) \\
& <\frac{A_{\min }}{2}\left(\frac{r_{\text {min }}}{K_{\text {max }}^{2}}\left(A_{\text {min }}-\frac{A_{\text {min }}}{2}\right)\left(\frac{A_{\text {min }}}{2}-A_{\text {min }}\right)-h_{\text {min }}\right) \\
& =\frac{A_{\text {min }}}{2}\left(-\frac{r_{\text {min }}}{K_{\text {max }}^{2}}\left(\frac{A_{\text {min }}}{2}\right)^{2}-h_{\text {min }}\right)<0 .
\end{aligned}
$$

and

$$
\begin{aligned}
\dot{N} & =f\left(t,-\frac{A_{\text {min }}}{2}\right)=\left(-\frac{A_{\min }}{2}\right)\left(\frac{r(t)}{K^{2}(t)}\left(K(t)+\frac{A_{\min }}{2}\right)\left(-\frac{A_{\min }}{2}-A(t)\right)-h(t)\right) \\
& >\left(-\frac{A_{\min }}{2}\right)\left(\frac{r_{\min }}{K_{\text {max }}^{2}}\left(A_{\min }+\frac{A_{\min }}{2}\right)\left(-\frac{A_{\min }}{2}-A_{\min }\right)-h_{\text {max }}\right) \\
& =\left(-\frac{A_{\min }}{2}\right)\left(-\frac{9}{4} \frac{r_{\text {min }} A_{\min }^{2}}{K_{\max }^{2}}-h_{\max }\right)>0,
\end{aligned}
$$

since $h_{\max }>h_{\min }>-\frac{1}{4} r_{\min }\left(\frac{A_{\min }}{K_{\max }}\right)^{2}>-\frac{9}{4} r_{\min }\left(\frac{A_{\min }}{K_{\max }}\right)^{2}$.

The conclusion that $\beta_{0}(t)=\frac{A_{\min }}{2}$ and $\alpha_{0}(t)=-\frac{A_{\min }}{2}$ are strict upper and lower fences, respectively and $\alpha_{0}(t)<\beta_{0}(t)$. The trivial solution $N_{\text {triv }}(t)$ to Eq. (1.7) is located in a funnel bounded by the horizontal lines $\alpha_{0}(t)$ and $\beta_{0}(t)$. The two horizontal lines, $\beta_{2}(t)=\frac{A_{\min }}{2}$ and $\alpha_{2}(t)=\frac{A_{\max }+K_{\min }}{2}$ are strict upper and lower fences, respectively. They form an antifunnel and $\alpha_{2}(t)>\beta_{2}(t)$ as $t \rightarrow+\infty$. For an unstable periodic solution $N_{2}(t)$, as $t \rightarrow+\infty$ to Eq. (1.7) contained in the antifunnel and the other solutions with close initial data in the antifunnel. Therefore, we have established existence of three periodic solutions to Eq. (1.7), namely the trivial solution $N_{\text {triv }}(t)$ and two positive solutions, a stable solution $N_{1}(t)$ and an unstable solution $N_{2}(t)$ satisfying, for all $t \in[0,+\infty)$, 


$$
-\frac{A_{\min }}{2}<N_{\text {triv }}(t)<\frac{A_{\min }}{2}<N_{2}(t)<\frac{A_{\max }+K_{\min }}{2}<N_{1}(t)<A_{\min }+K_{\max } .
$$

b. Let $N=2 K_{\max }$, for all $t \geq t_{0}$,

$$
\begin{aligned}
\dot{N} & =f\left(t, 2 K_{\text {max }}\right)=\left(2 K_{\text {max }}\right)\left(\frac{r(t)}{K^{2}(t)}\left(K(t)-2 K_{\text {max }}\right)\left(2 K_{\text {max }}-A(t)\right)-h(t)\right) \\
& <\left(2 K_{\text {max }}\right)\left(\frac{r_{\text {min }}}{K_{\text {max }}^{2}}\left(K_{\text {max }}-2 K_{\text {max }}\right)\left(2 K_{\text {max }}-K_{\text {max }}\right)-h_{\text {min }}\right) \\
& =\left(2 K_{\text {max }}\right)\left(-r_{\text {min }}-h_{\text {min }}\right)<0 .
\end{aligned}
$$

On the other hand,

$$
\begin{aligned}
\dot{N} & =f\left(t, K_{\text {min }}\right)=\left(K_{\text {min }}\right)\left(\frac{r(t)}{K^{2}(t)}\left(K(t)-K_{\text {min }}\right)\left(K_{\text {min }}-A(t)\right)-h(t)\right) \\
& >\left(K_{\text {min }}\right)\left(\frac{r_{\text {min }}}{K_{\text {max }}^{2}}\left(K_{\text {max }}-K_{\text {min }}\right)\left(K_{\text {min }}-A_{\text {max }}\right)-h_{\text {max }}\right)>0 .
\end{aligned}
$$

The existence of the periodic solution $N_{1}(t)$ is guaranteed by Theorem (2.3.1) and Theorem (2.3.4). There is a funnel that contains an asymptotically stable periodic solution $N_{1}(t)$ and all other solutions with close initial data to Eq. (1.7), these solutions stay in the funnel bounded by the horizontal lines $\beta_{1}(t)=2 K_{\max }$, $\alpha_{1}(t)=K_{\min }$ and $\alpha_{1}(t)<\beta_{1}(t)$. Then, observe that,

$$
\begin{aligned}
\dot{N} & =f\left(t,-r_{\text {max }}\right)=\left(-r_{\text {max }}\right)\left(\frac{r(t)}{K^{2}(t)}\left(K(t)+r_{\text {max }}\right)\left(-r_{\text {max }}-A(t)\right)-h(t)\right) \\
& <\left(-r_{\text {max }}\right)\left(\frac{r_{\text {max }}}{K_{\text {max }}^{2}}\left(K_{\text {max }}+r_{\text {max }}\right)\left(-r_{\text {max }}-A_{\text {max }}\right)-h_{\text {max }}\right) \\
& <\left(r_{\text {max }}\right)\left(r_{\text {max }}\left(\frac{A_{\text {max }}+r_{\text {max }}}{K_{\text {max }}}\right)^{2}+h_{\text {max }}\right)<0 .
\end{aligned}
$$

and 


$$
\begin{aligned}
\dot{N} & =f\left(t,-K_{\text {max }}\right)=\left(-K_{\text {max }}\right)\left(\frac{r(t)}{K^{2}(t)}\left(K(t)+K_{\text {max }}\right)\left(-K_{\text {max }}-A(t)\right)-h(t)\right) \\
& >\left(-K_{\text {max }}\right)\left(\frac{r_{\text {min }}}{K_{\text {max }}^{2}}\left(K_{\text {max }}+K_{\text {max }}\right)\left(-K_{\text {max }}-A_{\text {max }}\right)-h_{\text {max }}\right) \\
& =\left(K_{\text {max }}\right)\left(\frac{r_{\text {min }}}{K_{\text {max }}^{2}}\left(2 K_{\text {max }}\right)\left(K_{\text {max }}+A_{\text {max }}\right)+h_{\text {max }}\right) \\
& >\left(K_{\text {max }}\right)\left(4 r_{\text {min }}+h_{\text {max }}\right)>0 .
\end{aligned}
$$

The two horizontal lines $\beta_{0}(t)=-r_{\max }$ and $\alpha_{0}(t)=K_{\min }$ are strict upper and lower fences, respectively. They form an antifunnel and $\alpha_{0}(t)>\beta_{0}(t)$ as $t \rightarrow+\infty$. The trivial solution $N_{\text {triv }}(t)$ to Eq. (1.7) is unstable between the horizontal lines $\alpha_{0}(t)$ and $\beta_{0}(t)$. Consequently, Eq. (1.7) has an asymptotically stable periodic solution $N_{2}(t)$. There is a funnel that contains an asymptotically stable periodic solution $N_{2}(t)$ and all other solutions with close initial data to Eq. (1.7), these solutions stay in the funnel bounded by the horizontal lines $\beta_{2}(t)=-r_{\max }, \alpha_{2}(t)=-K_{\max }$ and $\alpha_{2}(t)<\beta_{2}(t)$. Therefore, we have established existence of three periodic solutions to Eq. (1.7), namely the trivial solution $N_{\text {triv }}(t)$ and the positive and negative solutions $N_{1}(t)$ and $N_{2}(t)$, which are asymptotically stable, for all $t \in \mathbb{R}$,

$$
-K_{\max }<N_{2}(t)<-r_{\max }<N_{\text {triv }}(t)<K_{\min }<N_{1}(t)<2 K_{\max } .
$$

The proof is completed. 


\subsubsection{Existence of Periodic Solutions and their Stability for $A(t)<0$ and $h(t)>0$}

\section{( Harvesting for Weak Allee Effect )}

Theorem 4.3 Let $A(t)<0$ and $h(t)>0$ and harvesting satisfies the condition

a. $\max _{t \geq 0}\left\{-\frac{A_{\min } r_{\min }}{K_{\max }}, \frac{3}{4} r_{\min }\left(\frac{A_{\max }}{K_{\max }}\right)^{2}\right\}<h_{\min }<h_{\max }<\frac{1}{4} r_{\min }\left(\frac{K_{\min }}{K_{\max }}\right)^{2}$ then Eq. (1.7) has three periodic solutions; the asymptotically stable trivial solution $N_{\text {triv }}(t)$ and two positive solutions, asymptotically stable solution $N_{1}(t)$ and an unstable solution $N_{2}(t)$.

b. $h_{\max }<\min _{t \geq 0}\left\{-\frac{A_{\max } r_{\max }}{K_{\min }}, \frac{3}{4} r_{\min }\left(\frac{A_{\max }}{K_{\max }}\right)^{2}\right\}$ then Eq. (1.7) has three periodic solutions; the trivial solution $N_{\text {triv }}(t)$ which is unstable a positive solutions $N_{1}(t)$ and a negative solution $N_{2}(t)$, both asymptotically stable.

Proof.

a. Let $N=K_{\text {max }}$, then

$$
\begin{aligned}
\dot{N} & =f\left(t, K_{\max }\right)=\left(K_{\max }\right)\left(\frac{r(t)}{K^{2}(t)}\left(K(t)-K_{\max }\right)\left(K_{\max }-A(t)\right)-h(t)\right) \\
& <\left(K_{\max }\right)\left(\frac{r_{\text {min }}}{K_{\max }^{2}}\left(K_{\max }-K_{\max }\right)\left(K_{\max }-A_{\max }\right)-h_{\min }\right) \\
& =-K_{\max } h_{\min }<0 .
\end{aligned}
$$

On the other hand,

$$
\begin{aligned}
\dot{N} & =f\left(t, \frac{K_{\text {min }}}{2}\right)=\left(\frac{K_{\text {min }}}{2}\right)\left(\frac{r(t)}{K^{2}(t)}\left(K(t)-\frac{K_{\text {min }}}{2}\right)\left(\frac{K_{\text {min }}}{2}-A(t)\right)-h(t)\right) \\
& >\left(\frac{K_{\text {min }}}{2}\right)\left(\frac{r_{\text {min }}}{K_{\text {max }}^{2}}\left(K_{\text {min }}-\frac{K_{\text {min }}}{2}\right)\left(\frac{K_{\text {min }}}{2}\right)-h_{\text {max }}\right) \\
& =\left(\frac{K_{\text {min }}}{2}\right)\left(\frac{1}{4} r_{\text {min }}\left(\frac{K_{\text {min }}}{K_{\text {max }}}\right)^{2}-h_{\text {max }}\right)>0 .
\end{aligned}
$$


Therefore, there is a funnel that contains an asymptotically stable periodic solution $N_{1}(t)$ and all other solutions with close initial data to Eq. (1.7). These solutions stay in the funnel bounded by the horizontal lines $\beta_{1}(t)=K_{\max }, \quad \alpha_{1}(t)=\frac{K_{\min }}{2}$ and $\alpha_{1}(t)<\beta_{1}(t)$. Next, we observe that,

$$
\begin{aligned}
\dot{N} & =f\left(t,-\frac{A_{\max }}{2}\right)=\left(-\frac{A_{\max }}{2}\right)\left(\frac{r(t)}{K^{2}(t)}\left(K(t)+\frac{A_{\max }}{2}\right)\left(-\frac{A_{\max }}{2}-A(t)\right)-h(t)\right) \\
& <\left(-\frac{A_{\max }}{2}\right)\left(\frac{r_{\min }}{K_{\text {max }}^{2}}\left(-A_{\max }+\frac{A_{\text {max }}}{2}\right)\left(-\frac{A_{\max }}{2}-A_{\max }\right)-h_{\text {min }}\right) \\
& =\left(-\frac{A_{\max }}{2}\right)\left(\frac{3}{4} r_{\min }\left(\frac{A_{\max }}{K_{\text {max }}}\right)^{2}-h_{\text {min }}\right)<0 .
\end{aligned}
$$

and

$$
\begin{aligned}
\dot{N} & =f\left(t, 2 A_{\max }\right)=\left(2 A_{\max }\right)\left(\frac{r(t)}{K^{2}(t)}\left(K(t)-2 A_{\max }\right)\left(2 A_{\max }-A(t)\right)-h(t)\right) \\
& >\left(2 A_{\max }\right)\left(\frac{r_{\text {min }}}{K_{\text {max }}^{2}}\left(A_{\max }-2 A_{\max }\right)\left(2 A_{\text {max }}-A_{\text {max }}\right)-h_{\text {max }}\right) \\
& =\left(2 A_{\max }\right)\left(-r_{\text {min }}\left(\frac{A_{\text {max }}}{K_{\text {max }}}\right)^{2}-h_{\text {max }}\right)>0 .
\end{aligned}
$$

The trivial solution $N_{\text {triv }}(t)$ to Eq. (1.7) is located in a funnel bounded by the horizontal lines $\beta_{0}(t)=-\frac{A_{\max }}{2}, \alpha_{0}(t)=2 A_{\max }$ and $\alpha_{0}(t)<\beta_{0}(t)$, this solution is asymptotically stable. The two horizontal lines $\beta_{2}(t)=-\frac{A_{\max }}{2}$ and $\alpha_{2}(t)=\frac{K_{\min }}{2}$ are strict upper and lower fences, respectively. They form an antifunnel, and the fact that $\alpha_{2}(t)>\beta_{2}(t)$ as $t \rightarrow+\infty$. Consequently, Eq. (1.7) has an unstable periodic solution $N_{2}(t)$, as $t \rightarrow+\infty$, between the horizontal lines $\alpha_{2}(t)$ and $\beta_{2}(t)$. Therefore, we have established existence of three periodic solutions to Eq. (1.7). 


$$
2 A_{\max }<N_{\text {triv }}(t)<-\frac{A_{\max }}{2}<N_{2}(t)<\frac{K_{\min }}{2}<N_{1}(t)<K_{\max } .
$$

b. Let $N=K_{\text {max }}$, then $\dot{N}=f\left(t, K_{\text {max }}\right)<0$, this is shown in part (a).

On the other hand,

$$
\begin{aligned}
\dot{N} & =f\left(t,-\frac{A_{\max }}{2}\right)=\left(-\frac{A_{\max }}{2}\right)\left(\frac{r(t)}{K^{2}(t)}\left(K(t)+\frac{A_{\max }}{2}\right)\left(-\frac{A_{\max }}{2}-A(t)\right)-h(t)\right) \\
& >\left(-\frac{A_{\max }}{2}\right)\left(\frac{r_{\min }}{K_{\max }^{2}}\left(-A_{\max }+\frac{A_{\max }}{2}\right)\left(-\frac{A_{\max }}{2}-A_{\max }\right)-h_{\max }\right) \\
& =\left(-\frac{A_{\max }}{2}\right)\left(\frac{3}{4} r_{\min }\left(\frac{A_{\max }}{K_{\max }}\right)^{2}-h_{\max }\right)>0 .
\end{aligned}
$$

Consequently, Eq. (1.7) has an asymptotically stable periodic solution $N_{1}(t)$. There is a funnel that contains an asymptotically stable periodic solution $N_{1}(t)$ and all other solutions with close initial data to Eq. (1.7). These solutions stay in the funnel bounded by the horizontal lines $\beta_{1}(t)=K_{\max }, \quad \alpha_{1}(t)=-\frac{A_{\max }}{2}$, and $\alpha_{1}(t)<\beta_{1}(t)$. Next, observe that,

$$
\begin{aligned}
\dot{N} & =f\left(t, \frac{A_{\max }}{2}\right)=\left(\frac{A_{\max }}{2}\right)\left(\frac{r(t)}{K^{2}(t)}\left(K(t)-\frac{A_{\max }}{2}\right)\left(\frac{A_{\max }}{2}-A(t)\right)-h(t)\right) \\
& <\left(\frac{A_{\max }}{2}\right)\left(\frac{r_{\min }}{K_{\max }^{2}}\left(A_{\max }-\frac{A_{\max }}{2}\right)\left(\frac{A_{\max }}{2}+A_{\max }\right)-h_{\max }\right) \\
& <\left(\frac{A_{\max }}{2}\right)\left(\frac{3}{4} r_{\min }\left(\frac{A_{\max }}{K_{\max }}\right)^{2}-h_{\max }\right)<0,
\end{aligned}
$$

and 


$$
\begin{aligned}
& \dot{N}=f\left(t, 2 A_{\text {min }}\right)=\left(2 A_{\text {min }}\right)\left(\frac{r(t)}{K^{2}(t)}\left(K(t)-2 A_{\text {min }}\right)\left(2 A_{\text {min }}-A(t)\right)-h(t)\right) \\
& >\left(2 A_{\min }\right)\left(\frac{r_{\min }}{K_{\text {max }}^{2}}\left(A_{\min }-2 A_{\min }\right)\left(2 A_{\min }-A_{\min }\right)-h_{\text {max }}\right) \\
& =\left(2 A_{\min }\right)\left(-r_{\min }\left(\frac{A_{\min }}{K_{\max }}\right)^{2}-h_{\max }\right)>0 \text {. }
\end{aligned}
$$

The trivial solution $N_{\text {triv }}(t)$ to Eq. (1.7) is an unstable bounded by two horizontal lines $\beta_{0}(t)=\frac{A_{\max }}{2}$ and $\alpha_{0}(t)=-\frac{A_{\max }}{2}$ are strict upper and lower fences, respectively. They form an antifunnel, since the fact that $\alpha_{0}(t)>\beta_{0}(t)$ as $t \rightarrow+\infty$. Because of, Eq. (1.7) has an asymptotically stable periodic solution $N_{2}(t)$ for all $t \in[0,+\infty)$. There is a funnel that contains an asymptotically stable periodic solution $N_{2}(t)$ and all other solutions with close initial data to Eq. (1.7). These solutions stay in the funnel bounded by the horizontal lines $\beta_{2}(t)=\frac{A_{\max }}{2}, \alpha_{2}(t)=2 A_{\min }$, and $\alpha_{2}(t)<\beta_{2}(t)$. Therefore, we have established existence of three periodic solutions to Eq. (1.7),

$$
2 A_{\min }<N_{2}(t)<\frac{A_{\max }}{2}<N_{\text {triv }}(t)<-\frac{A_{\max }}{2}<N_{1}(t)<K_{\max } .
$$

The proof is completed.

\subsubsection{Existence of Periodic Solutions and their Stability for $A(t)<0$ and $h(t)<0$}

\section{( Stocking for Weak Allee Effect )}

Theorem 4.4 Let $A(t)<0$ and $h(t)<0$ and harvesting satisfies the condition $h_{\max }>\max _{t \geq 0}\left\{-r_{\min },-4 r_{\min }\left(\frac{K_{\min }}{K_{\max }}\right)^{2}\right\}$ then Eq. (1.7) has three periodic solutions; the trivial solution $N_{\text {triv }}(t)$ which is unstable a positive solutions $N_{1}(t)$ and a negative 
solution $N_{2}(t)$, both asymptotically stable.

Proof. Let $N=2 K_{\max }$, for all $t \geq t_{0}$

$$
\begin{aligned}
\dot{N} & =f\left(t, 2 K_{\text {max }}\right)=\left(2 K_{\text {max }}\right)\left(\frac{r(t)}{K^{2}(t)}\left(K(t)-2 K_{\text {max }}\right)\left(2 K_{\text {max }}-A(t)\right)-h(t)\right) \\
& <\left(2 K_{\text {max }}\right)\left(\frac{r_{\text {min }}}{K_{\text {max }}^{2}}\left(K_{\text {max }}-2 K_{\text {max }}\right)\left(2 K_{\text {max }}-K_{\text {max }}\right)-h_{\text {max }}\right) \\
& =\left(2 K_{\text {max }}\right)\left(-r_{\text {min }}-h_{\text {max }}\right)<0 .
\end{aligned}
$$

On the other hand,

$$
\begin{aligned}
\dot{N} & =f\left(t, \frac{K_{\min }}{2}\right)=\left(\frac{K_{\min }}{2}\right)\left(\frac{r(t)}{K^{2}(t)}\left(K(t)-\frac{K_{\min }}{2}\right)\left(\frac{K_{\min }}{2}-A(t)\right)-h(t)\right) \\
& >\left(\frac{K_{\min }}{2}\right)\left(\frac{r_{\min }}{K_{\max }^{2}}\left(K_{\min }-\frac{K_{\min }}{2}\right)\left(\frac{K_{\min }}{2}-A_{\max }\right)-h_{\min }\right) \\
& =\left(\frac{K_{\min }}{2}\right)\left(\frac{r_{\min }}{K_{\max }^{2}}\left(\frac{K_{\min }}{2}\right)\left(\frac{K_{\min }}{2}-A_{\max }\right)-h_{\min }\right)>0 .
\end{aligned}
$$

Therefore, Eq. (1.7) has an asymptotically stable periodic solution $N_{1}(t)$. There is a funnel that contains an asymptotically stable periodic solution $N_{1}(t)$ and all other solutions with close initial data to Eq. (1.7). These solutions stay in the funnel bounded by the horizontal lines $\beta_{1}(t)=2 K_{\max }, \alpha_{1}(t)=\frac{K_{\min }}{2}$, and $\alpha_{1}(t)<\beta_{1}(t)$.

There is a funnel that contains an asymptotically stable periodic solution $N_{1}(t)$ to Eq. (1.7). Then, we observe that,

$$
\begin{aligned}
\dot{N} & =f\left(t, \frac{A_{\max }}{2}\right)=\left(\frac{A_{\max }}{2}\right)\left(\frac{r(t)}{K^{2}(t)}\left(K(t)-\frac{A_{\max }}{2}\right)\left(\frac{A_{\max }}{2}-A(t)\right)-h(t)\right) \\
& <\left(\frac{A_{\max }}{2}\right)\left(\frac{r_{\min }}{K_{\max }^{2}}\left(-A_{\max }-\frac{A_{\max }}{2}\right)\left(\frac{A_{\max }}{2}-A_{\max }\right)-h_{\max }\right) \\
& =\left(\frac{A_{\max }}{2}\right)\left(\frac{3}{4} r_{\min }\left(\frac{A_{\max }}{K_{\max }}\right)^{2}-h_{\max }\right)<0 .
\end{aligned}
$$

and 


$$
\begin{aligned}
\dot{N}=f\left(t,-K_{\text {min }}\right) & =\left(-K_{\text {min }}\right)\left(\frac{r(t)}{K^{2}(t)}\left(K(t)+K_{\text {min }}\right)\left(-K_{\text {min }}-A(t)\right)-h(t)\right) \\
& >\left(-K_{\text {min }}\right)\left(\frac{r_{\text {min }}}{K_{\text {max }}^{2}}\left(K_{\text {min }}+K_{\text {min }}\right)\left(-K_{\text {min }}-K_{\text {min }}\right)-h_{\text {min }}\right) \\
& =\left(-K_{\text {min }}\right)\left(-4 r_{\text {min }}\left(\frac{K_{\text {min }}}{K_{\text {max }}}\right)^{2}-h_{\text {min }}\right)>0 .
\end{aligned}
$$

Therefore, the trivial solution $N_{\text {triv }}(t)$ to Eq. (1.7) is an unstable bounded by two horizontal lines $\beta_{0}(t)=\frac{A_{\max }}{2}$ and $\alpha_{0}(t)=\frac{K_{\min }}{2}$ are strict upper and lower fences, respectively. They form an antifunnel, because of the fact $\alpha_{0}(t)>\beta_{0}(t)$ as $t \rightarrow+\infty$. Also, Eq. (1.7) has an asymptotically stable periodic solution $N_{2}(t)$ for all $t \in[0,+\infty)$. There is a funnel that contains an asymptotically stable periodic solution $N_{2}(t)$ and all other solutions with close initial data to Eq. (1.7). These solutions stay in the funnel bounded by the horizontal lines $\beta_{2}(t)=\frac{A_{\max }}{2}, \alpha_{2}(t)=-K_{\min }$, and $\alpha_{2}(t)<\beta_{2}(t)$. Therefore, we have determined existence of three periodic solutions to Eq. (1.7),

$$
-K_{\min }<N_{2}(t)<\frac{A_{\max }}{2}<N_{t r i v}(t)<\frac{K_{\min }}{2}<N_{1}(t)<2 K_{\max } .
$$

The proof is completed.

4.1.5 Existence of Periodic Solutions and their Stability for $A(t)=0, h(t)>0$ and $A(t)=0, h(t)<0$ ( Harvesting and Stocking for Weak Allee Effect $)$

\section{Theorem 4.5}

a. Let $A(t)=0$ and $h(t)>0$ and harvesting satisfies the condition $h_{\max }<\frac{1}{4} r_{\min }\left(\frac{K_{\min }}{K_{\max }}\right)^{2}$ then Eq. (1.7) has three periodic solutions; the 
asymptotically stable trivial solution $N_{\text {triv }}(t)$ and two positive solutions, asymptotically stable solution $N_{1}(t)$ and an unstable solution $N_{2}(t)$.

b. Let $A(t)=0$ and $h(t)<0$ and harvesting satisfies the condition $h_{\max }<-\frac{3}{4} r_{\min }\left(\frac{K_{\min }}{K_{\max }}\right)^{2}$ then Eq. (1.7) has three periodic solutions; the trivial solution $N_{\text {triv }}(t)$ which is unstable a positive solution $N_{1}(t)$ and a negative solution $N_{2}(t)$, both asymptotically stable.

Proof.

a. Let $N=K_{\max }$, for all $t \geq t_{0}$ then

$$
\begin{aligned}
\dot{N} & =f\left(t, K_{\text {max }}\right)=\left(K_{\text {max }}\right)\left(\frac{r(t)}{K^{2}(t)} K_{\text {max }}\left(K(t)-K_{\text {max }}\right)-h(t)\right) \\
& <\left(K_{\text {max }}\right)\left(\frac{r_{\text {min }}}{K_{\text {max }}^{2}} K_{\text {max }}\left(K_{\text {max }}-K_{\text {max }}\right)-h_{\text {min }}\right) \\
& =-K_{\text {max }} h_{\text {min }}<0
\end{aligned}
$$

On the other hand,

$$
\begin{aligned}
& \dot{N}=f\left(t, \frac{K_{\min }}{2}\right)=\left(\frac{K_{\min }}{2}\right)\left(\frac{r(t)}{K^{2}(t)} \frac{K_{\min }}{2}\left(K(t)-\frac{K_{\min }}{2}\right)-h(t)\right) \\
& >\left(\frac{K_{\min }}{2}\right)\left(\frac{r_{\min }}{K_{\max }^{2}} \frac{K_{\min }}{2}\left(K_{\min }-\frac{K_{\min }}{2}\right)-h_{\max }\right) \\
& =\left(\frac{K_{\min }}{2}\right)\left(\frac{1}{4} r_{\min }\left(\frac{K_{\min }}{K_{\max }}\right)^{2}-h_{\max }\right)>0 \text {. }
\end{aligned}
$$

Consequently, Eq. (1.7) has an asymptotically stable periodic solution $N_{1}(t)$. There is a funnel that contains an asymptotically stable periodic solution $N_{1}(t)$ and all other solutions with close initial data to Eq. (1.7). These solutions stay in the funnel 
bounded by the horizontal lines $\beta_{1}(t)=K_{\max }, \alpha_{1}(t)=\frac{K_{\min }}{2}$ and $\alpha_{1}(t)<\beta_{1}(t)$.

There is a funnel that contains an asymptotically stable periodic solution $N_{1}(t)$ to Eq. (1.7). Then, we assume that $N=h_{\min }$,

$$
\begin{aligned}
\dot{N} & =f\left(t, h_{\text {min }}\right)=\left(h_{\text {min }}\right)\left(\frac{r(t)}{K^{2}(t)}\left(h_{\text {min }}\right)\left(K(t)-h_{\text {min }}\right)-h(t)\right) \\
& <\left(h_{\text {min }}\right)\left(\frac{r_{\text {min }}}{K_{\text {max }}^{2}}\left(h_{\text {min }}\right)\left(K_{\text {min }}-h_{\text {min }}\right)-h_{\text {min }}\right) \\
& <\left(h_{\text {min }}\right)^{2}\left(\frac{r_{\text {min }}}{K_{\text {max }}^{2}}\left(K_{\text {min }}-h_{\text {min }}\right)-1\right)<0
\end{aligned}
$$

and

$$
\begin{aligned}
\dot{N} & =f\left(t,-\frac{K_{\text {min }}}{2}\right)=\left(-\frac{K_{\min }}{2}\right)\left(\frac{r(t)}{K^{2}(t)}\left(-\frac{K_{\min }}{2}\right)\left(K(t)+\frac{K_{\min }}{2}\right)-h(t)\right) \\
& >\left(-\frac{K_{\text {min }}}{2}\right)\left(\frac{r_{\text {min }}}{K_{\text {max }}^{2}}\left(-\frac{K_{\text {min }}}{2}\right)\left(K_{\text {min }}+\frac{K_{\text {min }}}{2}\right)-h_{\text {max }}\right) \\
& =\left(\frac{K_{\min }}{2}\right)\left(\frac{3}{4} r_{\min }\left(\frac{K_{\min }}{K_{\text {max }}}\right)^{2}+h_{\text {max }}\right)>0 .
\end{aligned}
$$

So, the trivial solution $N_{\text {triv }}(t)$ to Eq. (1.7) is located in a funnel. This solution is asymptotical stable; all solutions enter a funnel bounded by the horizontal lines which are upper and lower fences $\beta_{0}(t)=h_{\min }, \alpha_{0}(t)=-\frac{K_{\min }}{2}$, and $\alpha_{0}(t)<\beta_{0}(t)$ respectively, and stay there. Consequently, Eq. (1.7) has an unstable periodic solution $N_{2}(t)$, as $t \rightarrow+\infty$, bounded by two horizontal lines $\beta_{2}(t)=h_{\min }$ and $\alpha_{2}(t)=\frac{K_{\min }}{2}$ are strict upper and lower fences, respectively, since the fact that $\alpha_{2}(t)>\beta_{2}(t)$ as $t \rightarrow+\infty$. Therefore, we have determined existence of three periodic solutions to Eq. (1.7), 


$$
-\frac{K_{\min }}{2}<N_{t r i v}(t)<h_{\min }<N_{2}(t)<\frac{K_{\min }}{2}<N_{1}(t)<K_{\max } .
$$

b. Let $N=2 K_{\max }$, then

$$
\begin{aligned}
\dot{N} & =f\left(t, 2 K_{\text {max }}\right)=\left(2 K_{\text {max }}\right)\left(\frac{r(t)}{K^{2}(t)}\left(2 K_{\text {max }}\right)\left(K(t)-2 K_{\text {max }}\right)-h(t)\right) \\
& <\left(2 K_{\text {max }}\right)\left(\frac{r_{\text {min }}}{K_{\text {max }}^{2}}\left(2 K_{\text {max }}\right)\left(K_{\text {max }}-2 K_{\text {max }}\right)-h_{\text {max }}\right) \\
& =\left(2 K_{\text {max }}\right)\left(-2 r_{\text {min }}-h_{\text {max }}\right)<0 .
\end{aligned}
$$

On the other hand, since $h_{\min }<0$, we have

$$
\begin{aligned}
\dot{N} & =f\left(t, K_{\text {min }}\right)=\left(K_{\text {min }}\right)\left(\frac{r(t)}{K^{2}(t)}\left(K_{\text {min }}\right)\left(K(t)-K_{\text {min }}\right)-h(t)\right) \\
& >\left(K_{\min }\right)\left(\frac{r_{\min }}{K_{\text {max }}^{2}}\left(K_{\min }\right)\left(K_{\min }-K_{\text {min }}\right)-h_{\text {min }}\right) \\
& =-K_{\min } h_{\text {min }}>0 .
\end{aligned}
$$

Thus, Eq. (1.7) has asymptotically stable periodic solution $N_{1}(t)$ to Eq. (1.7). There is a funnel that contains an asymptotically stable periodic solution $N_{1}(t)$ and all other solutions with close initial data to Eq. (1.7). These solutions stay in the funnel bounded by the horizontal lines $\beta_{1}(t)=2 K_{\max }$ and $\alpha_{1}(t)=K_{\min }$, since $\alpha_{1}(t)<\beta_{1}(t)$. Next, we observe that,

$$
\begin{aligned}
\dot{N} & =f\left(t,-\frac{K_{\text {min }}}{2}\right)=\left(-\frac{K_{\min }}{2}\right)\left(\frac{r(t)}{K^{2}(t)}\left(-\frac{K_{\min }}{2}\right)\left(K(t)+\frac{K_{\text {min }}}{2}\right)-h(t)\right) \\
& <\left(-\frac{K_{\text {min }}}{2}\right)\left(\frac{r_{\text {min }}}{K_{\text {max }}}\left(-\frac{K_{\text {min }}}{2}\right)\left(K_{\text {min }}+\frac{K_{\text {min }}}{2}\right)-h_{\text {max }}\right) \\
& <\left(-\frac{K_{\text {min }}}{2}\right)\left(-\frac{3}{4} r_{\text {min }}\left(\frac{K_{\min }}{K_{\text {max }}}\right)^{2}-h_{\text {max }}\right)<0
\end{aligned}
$$

and since $h_{\text {min }}<0$, 


$$
\begin{aligned}
\dot{N} & =f\left(t,-K_{\text {max }}\right)=\left(-K_{\text {max }}\right)\left(\frac{r(t)}{K^{2}(t)}\left(-K_{\text {max }}\right)\left(K(t)+K_{\text {max }}\right)-h(t)\right) \\
& >\left(-K_{\text {max }}\right)\left(\frac{r_{\text {min }}}{K_{\text {max }}^{2}}\left(-K_{\text {max }}\right)\left(K_{\text {max }}+K_{\text {max }}\right)-h_{\text {min }}\right) \\
& =\left(-K_{\text {max }}\right)\left(-2 r_{\text {min }}-h_{\text {min }}\right)>0 .
\end{aligned}
$$

Consequently, the trivial solution $N_{\text {triv }}(t)$ to Eq. (1.7) is an unstable, this solution of the Eq. (1.7) stay in the antifunnel bounded by two horizontal lines $\beta_{0}(t)=K_{\min }$ and $\alpha_{0}(t)=-\frac{K_{\min }}{2}$ are strict upper and lower fences, respectively, since the fact that $\alpha_{0}(t)<\beta_{0}(t)$ as $t \rightarrow+\infty$. Because of, Eq. (1.7) has an asymptotically stable periodic solution $N_{2}(t)$ for all $t \rightarrow+\infty$. The asymptotically stable periodic solution $N_{1}(t)$ and all other solutions with close initial data to Eq. (1.7) stay in the funnel bounded by the horizontal lines $\beta_{2}(t)=-\frac{K_{\min }}{2}$, $\alpha_{2}(t)=-K_{\max }$, and $\alpha_{2}(t)<\beta_{2}(t)$. Therefore, we have established existence of three periodic solutions to Eq. (1.7), for all $t \in[0,+\infty)$, the corridors where these periodic solutions are located are

$$
-K_{\max }<N_{2}(t)<-\frac{K_{\min }}{2}<N_{t r i v}(t)<K_{\min }<N_{1}(t)<2 K_{\max }
$$

The proof is completed.

\subsection{Examples and Discussion}

In this section, we introduce the existence of the periodic solutions and their asymptotic behavior of Eq. (1.7) and also we investigate the effect of harvesting on the dynamics of population in a fluctuating environment described by Eq. (1.7). All the figures in this chapter have been plotted with the computer algebra system Mathematica. 
Example 4.6 Consider the following differential equation with $A(t)>0$ and $h(t)>0$,

$$
\begin{aligned}
\dot{N} & =(40+\sin 2 \pi t) N\left(1-\frac{N}{50+\cos 2 \pi t}\right) \\
& \times\left(\frac{N}{50+\cos 2 \pi t}-\gamma_{1} \frac{10+\sin 2 \pi t}{50+\cos 2 \pi t}\right)-\gamma_{2}(2+\cos 2 \pi t) N
\end{aligned}
$$

where

$r(t)=40+\sin 2 \pi t, K(t)=50+\cos 2 \pi t, A(t)=\gamma_{1}(10+\sin 2 \pi t), h(t)=\gamma_{2}(2+\cos 2 \pi t)$.

Let $\gamma_{1}=1$, and $\gamma_{2}=1$. Obviously, the condition (4.1) is satisfied $A_{\max }=11 \leq K_{\min }=49$. By the Theorem (4.1), the condition (4.3) is satisfied,

$$
\begin{aligned}
& h_{\max }<\frac{r_{\min }}{K_{\max }^{2}}\left(\frac{K_{\min }-A_{\max }}{2}\right)^{2} \\
& 3<\frac{39}{(51)^{2}}\left(\frac{49-11}{2}\right)^{2} \cong 5.41292 .
\end{aligned}
$$

Therefore, Eq. (4.8) has three periodic solutions: an asymptotically stable trivial solution $N_{\text {triv }}(t)=0$ and two positive solutions, an asymptotically stable solution $N_{1}(t)$ and an unstable solution $N_{2}(t)$.

By the Theorem (4.1), these three solutions satisfy the inequality,

$$
\begin{gathered}
-A_{\text {min }}<N_{\text {triv }}(t)<A_{\min }<N_{2}(t)<\frac{A_{\max }+K_{\min }}{2}<N_{1}(t)<K_{\max } \\
-9<N_{\text {triv }}(t)<9<N_{2}(t)<30<N_{1}(t)<51
\end{gathered}
$$

which are the corridors of these periodic solutions, see Figure 5. 


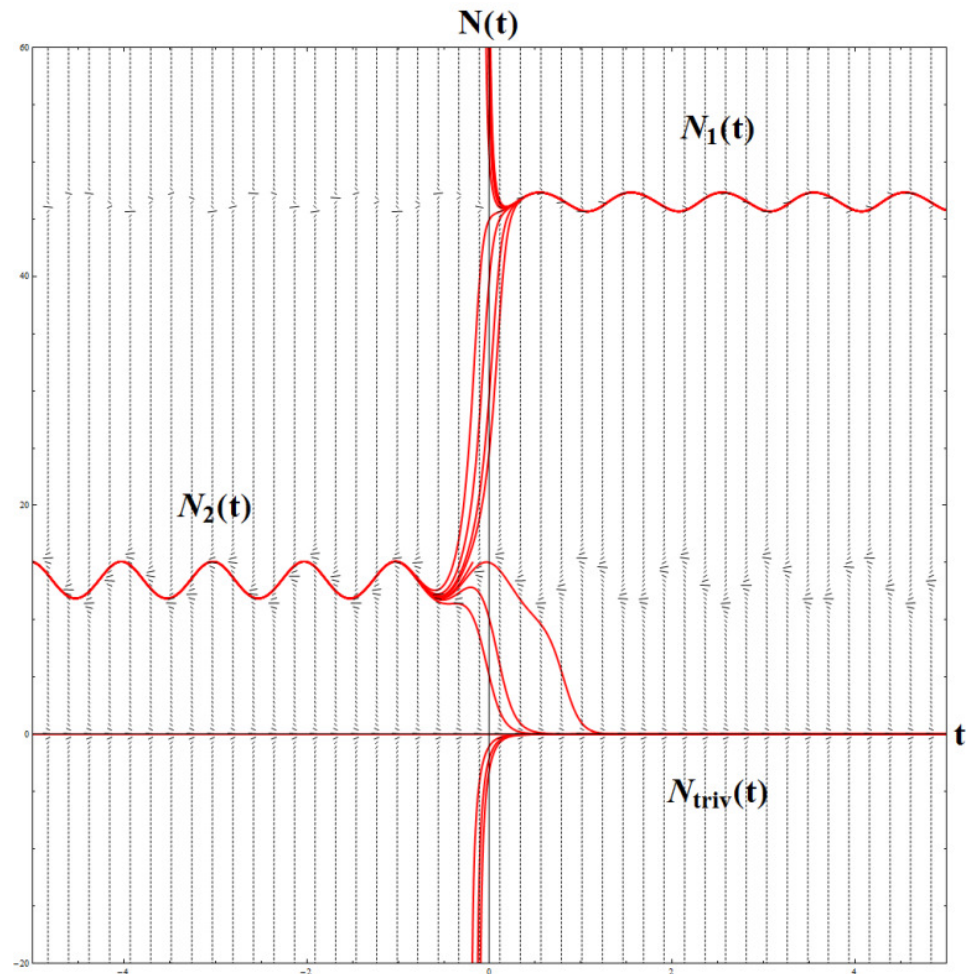

Figure 5: Existence of one positive asymptotically stable and one positive unstable periodic solution of Eq. (4.8) in case of strong Allee effect for $\gamma_{1}=1$ and harvesting for $\gamma_{2}=1$. 
Example 4.7 Consider the following differential equation with $A(t)>0$ and $h(t)<0$.

$$
\text { a. } \begin{aligned}
\dot{N} & =(10+\sin 2 \pi t) N\left(1-\frac{N}{10+\frac{1}{4} \cos 2 \pi t}\right) \\
& \times\left(\frac{N}{10+\frac{1}{4} \cos 2 \pi t}-\gamma_{1} \frac{8+\sin 2 \pi t}{10+\frac{1}{4} \cos 2 \pi t}\right)-\gamma_{2}\left(1+\frac{1}{100} \cos 2 \pi t\right) N
\end{aligned}
$$

where

$$
\begin{aligned}
& r(t)=10+\sin 2 \pi t, K(t)=10+\frac{1}{4} \cos 2 \pi t \\
& \begin{aligned}
& A(t)= \gamma_{1}(8+\sin 2 \pi t), h(t)=\gamma_{2}\left(1+\frac{1}{100} \cos 2 \pi t\right) . \\
& \text { b. } \dot{N}=(8+\sin 2 \pi t) N\left(1-\frac{N}{30+\cos 2 \pi t}\right) \\
& \quad \times\left(\frac{N}{30+\cos 2 \pi t}-\gamma_{1} \frac{2+\sin 2 \pi t}{30+\cos 2 \pi t}\right)-\gamma_{2}(5+\cos 2 \pi t) N
\end{aligned}
\end{aligned}
$$

where

$$
r(t)=8+\sin 2 \pi t, K(t)=30+\cos 2 \pi t, A(t)=\gamma_{1}(2+\sin 2 \pi t), h(t)=\gamma_{2}(5+\cos 2 \pi t) .
$$

a. Let $\gamma_{1}=1$, and $\gamma_{2}=-1$. Obviously, the condition (4.1) is satisfied $A_{\max }=9 \leq K_{\min }=10.25$. By the Theorem (4.2), the condition 


$$
\begin{aligned}
& h_{\min }>-\frac{1}{4} r_{\min }\left(\frac{A_{\min }}{K_{\max }}\right)^{2} \\
& -1.01>-\frac{1}{4}(9)\left(\frac{7}{10.25}\right)^{2}=-1.049375
\end{aligned}
$$

is satisfied. Thus, Eq. (4.9) has three periodic solutions: the asymptotically stable trivial solution $N_{\text {triv }}(t)=0$ and two positive solutions, asymptotically stable solution $N_{1}(t)$ and an unstable solution $N_{2}(t)$.

By the Theorem (4.2), these three solutions satisfy the inequality,

$$
\begin{gathered}
-\frac{A_{\min }}{2}<N_{\text {triv }}(t)<\frac{A_{\min }}{2}<N_{2}(t)<\frac{A_{\max }+K_{\min }}{2}<N_{1}(t)<A_{\min }+K_{\text {max }} . \\
-3.5<N_{\text {triv }}(t)<3.5<N_{2}(t)<9.375<N_{1}(t)<17.25
\end{gathered}
$$

which are the corridors of these periodic solutions, see Figure 6(a).

b. Let $\gamma_{1}=1$, and $\gamma_{2}=-1$. Obviously, the condition (4.1) is satisfied $A_{\max }=3 \leq K_{\min }=29$. By the Theorem (4.2), the condition

$$
\begin{aligned}
& -r_{\min }<h_{\min }<h_{\max }<-\left(r_{\max }\right)\left(\frac{A_{\max }+r_{\max }}{K_{\min }}\right)^{2} \\
& -7<-6<-4<-(9)\left(\frac{3+9}{29}\right)^{2}=-1.541022
\end{aligned}
$$

is satisfied. Therefore, Eq. (4.10) has three periodic solutions: the trivial solution $N_{\text {triv }}(t)=0$ which is unstable a positive solutions $N_{1}(t)$ and a negative solution $N_{2}(t)$, both asymptotically stable.

By the Theorem (4.2), these three solutions satisfy the inequality, 


$$
\begin{gathered}
-K_{\max }<N_{2}(t)<-r_{\max }<N_{t r i v}(t)<K_{\min }<N_{1}(t)<2 K_{\max } \\
-31<N_{2}(t)<-9<N_{\text {triv }}(t)<29<N_{1}(t)<62
\end{gathered}
$$

which are the corridors of these periodic solutions, see Figure 6(b). 


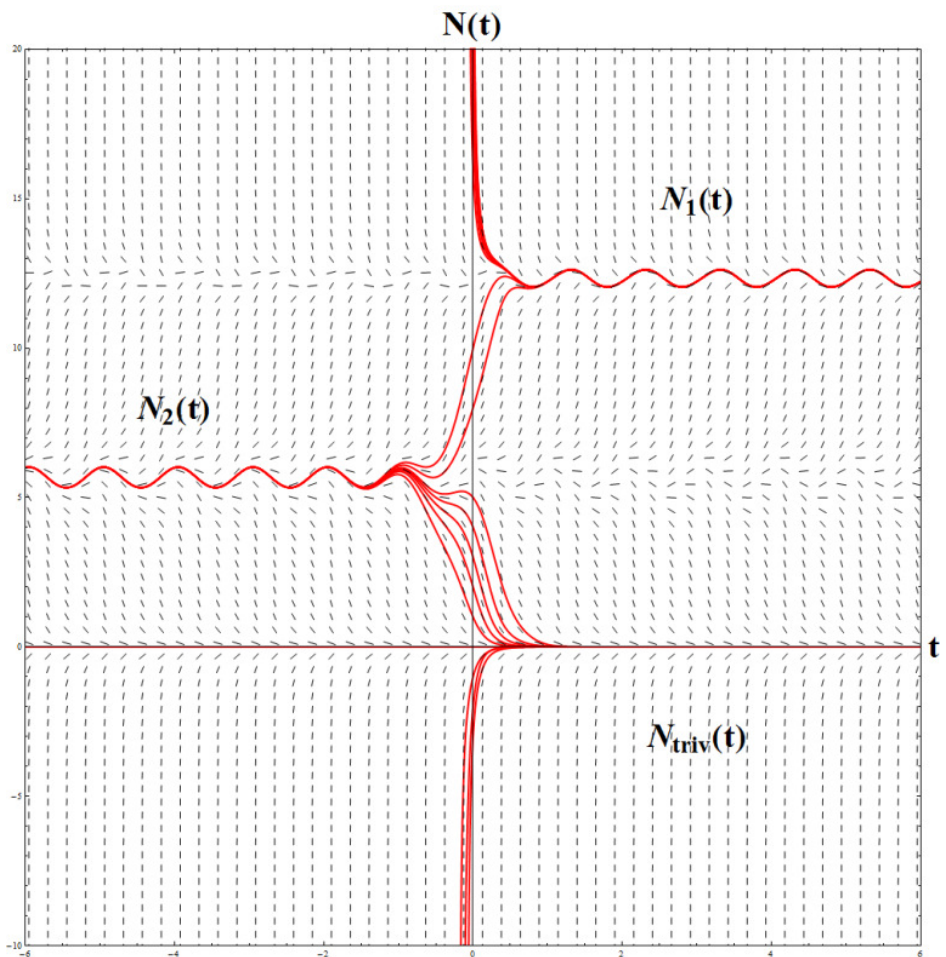

Figure 6(a): Existence of one positive asymptotically stable and one positive unstable periodic solution of Eq. (4.9) in case of strong Allee effect for $\gamma_{1}=1$ and stocking for $\gamma_{2}=-1$.

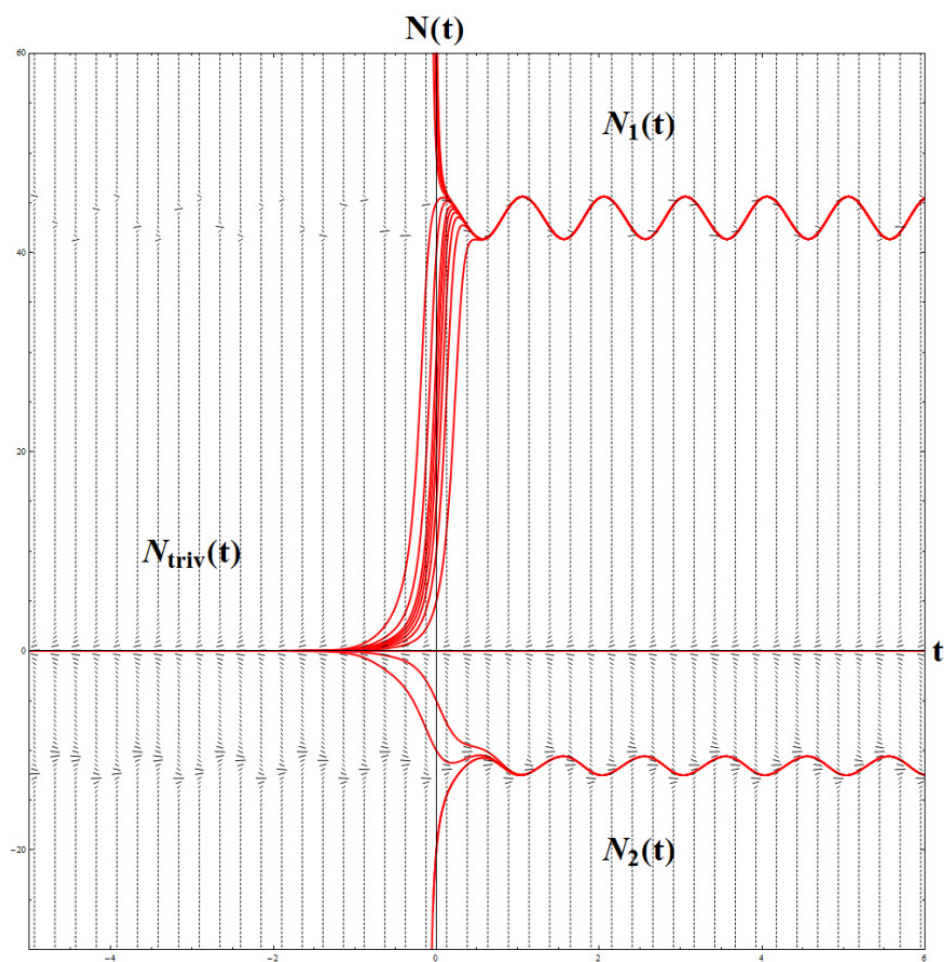

Figure 6(b): Existence of one positive asymptotically stable periodic solution of Eq. (4.10) in case of strong Allee effect for $\gamma_{1}=1$ and stocking for $\gamma_{2}=-1$. 
Example 4.8 Consider the following differential equation with $A(t)<0$ and $h(t)>0$,

a. $\dot{N}=(45+\sin 2 \pi t) N\left(1-\frac{N}{45+\cos 2 \pi t}\right)$

$$
\times\left(\frac{N}{45+\cos 2 \pi t}-\gamma_{1} \frac{2+\sin 2 \pi t}{45+\cos 2 \pi t}\right)-\gamma_{2}(8+\cos 2 \pi t) N
$$

where

$r(t)=45+\sin 2 \pi t, K(t)=45+\cos 2 \pi t, A(t)=\gamma_{1}(2+\sin 2 \pi t), h(t)=\gamma_{2}(8+\cos 2 \pi t)$.

b. $\dot{N}=(20+\sin 2 \pi t) N\left(1-\frac{N}{30+\cos 2 \pi t}\right)$

$$
\times\left(\frac{N}{30+\cos 2 \pi t}-\gamma_{1} \frac{17+\sin 2 \pi t}{30+\cos 2 \pi t}\right)-\gamma_{2}(2+\cos 2 \pi t) N
$$

where

$r(t)=20+\sin 2 \pi t, K(t)=30+\cos 2 \pi t, A(t)=\gamma_{1}(17+\sin 2 \pi t), h(t)=\gamma_{2}(2+\cos 2 \pi t)$.

a. Let $\gamma_{1}=-1$, and $\gamma_{2}=1$. Obviously, the condition (4.2) is satisfied $0<\left|A_{\min }\right|=3 \leq K_{\min }=44$. By the Theorem (4.3), the condition

$$
\max _{t \geq 0}\left\{-\frac{A_{\min } r_{\min }}{K_{\max }}, \frac{3}{4} r_{\min }\left(\frac{A_{\max }}{K_{\max }}\right)^{2}\right\}<h_{\min }<h_{\max }<\frac{1}{4} r_{\min }\left(\frac{K_{\min }}{K_{\max }}\right)^{2}
$$

$$
\max _{t \geq 0}\left\{-\frac{(-3)(44)}{46}, \frac{3}{4}(44)\left(\frac{-1}{46}\right)^{2}\right\}=2.869565<7<9<\frac{1}{4}(44)\left(\frac{44}{46}\right)^{2}=10.064272
$$


is satisfied. Thus, Eq. (4.11) has three periodic solutions: the asymptotically stable trivial solution $N_{\text {triv }}(t)=0$ and two positive solutions, asymptotically stable solution $N_{1}(t)$ and an unstable solution $N_{2}(t)$.

By the Theorem (4.3), these three solutions satisfy the inequality,

$$
\begin{aligned}
& 2 A_{\max }<N_{\text {triv }}(t)<-\frac{A_{\max }}{2}<N_{2}(t)<\frac{K_{\min }}{2}<N_{1}(t)<K_{\max } \\
& -2<N_{\text {triv }}(t)<0.5<N_{2}(t)<22<N_{1}(t)<46 .
\end{aligned}
$$

which are the corridors of these periodic solutions, see Figure 7(a).

b. Let $\gamma_{1}=-1$, and $\gamma_{2}=1$. Obviously, the condition (4.2) is satisfied $0<-A_{\max }=16 \leq K_{\min }=29$. By the Theorem (4.3), the condition

$$
\begin{gathered}
h_{\max }<\min _{t \geq 0}\left\{-\frac{A_{\max } r_{\max }}{K_{\min }}, \frac{3}{4} r_{\min }\left(\frac{A_{\max }}{K_{\max }}\right)^{2}\right\} \\
3<\min _{t \geq 0}\left\{-\frac{(-16)(21)}{29}, \frac{3}{4}(19)\left(\frac{-16}{31}\right)^{2}\right\} \cong 3.796046
\end{gathered}
$$

is satisfied. Therefore, Eq. (4.12) has three periodic solutions: the trivial solution $N_{\text {triv }}(t)=0$ which is unstable a positive solution $N_{1}(t)$ and a negative solution $N_{2}(t)$, both asymptotically stable.

By the Theorem (4.3), these three solutions satisfy the inequality,

$$
\begin{gathered}
2 A_{\text {min }}<N_{2}(t)<\frac{A_{\max }}{2}<N_{\text {triv }}(t)<-\frac{A_{\max }}{2}<N_{1}(t)<K_{\max } \\
-36<N_{2}(t)<-8<N_{\text {triv }}(t)<8<N_{1}(t)<31 .
\end{gathered}
$$

which are the corridors of these periodic solutions, see Figure 7(b). 


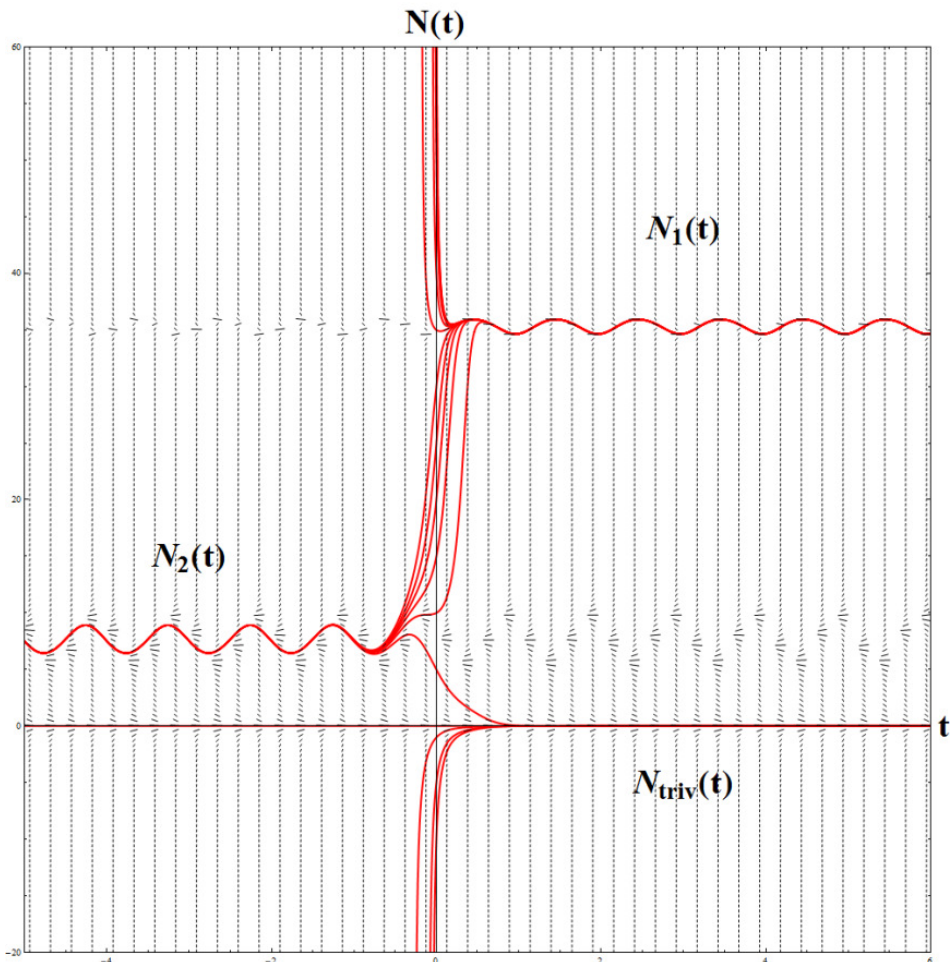

Figure 7(a): Existence of one positive asymptotically stable and one positive unstable periodic solution of Eq. (4.11) in case of weak Allee effect for $\gamma_{1}=-1$ and harvesting for $\gamma_{2}=1$.

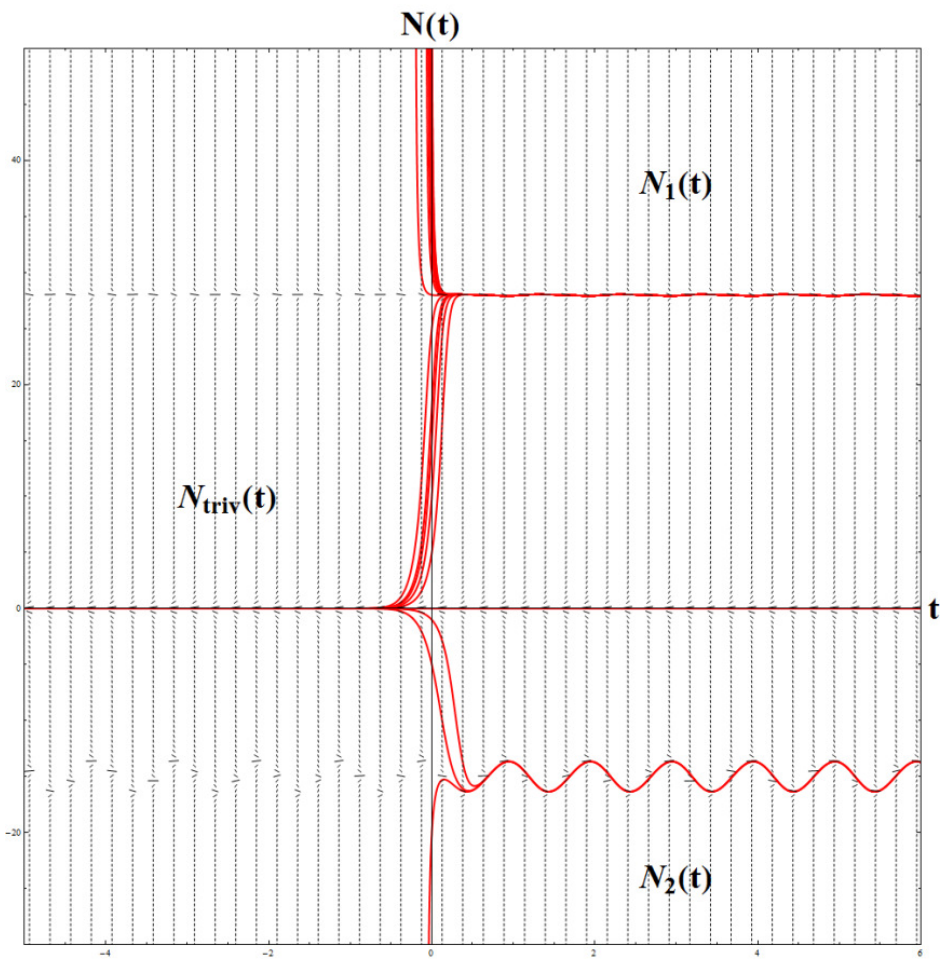

Figure 7(b): Existence of one positive asymptotically stable of Eq. (4.12) in case of weak Allee effect $\gamma_{1}=-1$ and harvesting $\gamma_{2}=1$. 
Example 4.9 Consider the following differential equation with $A(t)<0$ and

$$
\begin{aligned}
& h(t)<0, \\
& \qquad \begin{aligned}
\dot{N} & =(5+\sin 2 \pi t) N\left(1-\frac{N}{10+\cos 2 \pi t}\right) \\
& \times\left(\frac{N}{10+\cos 2 \pi t}-\gamma_{1} \frac{6+\sin 2 \pi t}{10+\cos 2 \pi t}\right)-\gamma_{2}(2+\cos 2 \pi t) N
\end{aligned}
\end{aligned}
$$

where

$$
r(t)=5+\sin 2 \pi t, K(t)=10+\cos 2 \pi t, A(t)=\gamma_{1}(6+\sin 2 \pi t), h(t)=\gamma_{2}(2+\cos 2 \pi t) .
$$

Let $\gamma_{1}=-1$, and $\gamma_{2}=-1$. The condition (4.2) is satisfied $0<\left|A_{\min }\right|=7 \leq K_{\min }=9$. By the Theorem (4.4), the condition

$$
\begin{aligned}
& h_{\max }>\max _{t \geq 0}\left\{-r_{\min },-4 r_{\min }\left(\frac{K_{\min }}{K_{\max }}\right)^{2}\right\} \\
& -1>\max _{t \geq 0}\left\{-4,-4(4)\left(\frac{9}{11}\right)^{2}\right\}=-4
\end{aligned}
$$

is satisfied. Therefore, Eq. (4.13) has three periodic solutions: the trivial solution $N_{\text {triv }}(t)=0$ which is unstable a positive solutions $N_{1}(t)$ and a negative solution $N_{2}(t)$, both asymptotically stable.

By the Theorem (4.4), these three solutions satisfy the inequality,

$$
\begin{gathered}
-K_{\min }<N_{2}(t)<\frac{A_{\max }}{2}<N_{\text {triv }}(t)<\frac{K_{\min }}{2}<N_{1}(t)<2 K_{\max } \\
-9<N_{2}(t)<-2.5<N_{\text {triv }}(t)<4.5<N_{1}(t)<22 .
\end{gathered}
$$

which are the corridors of these periodic solutions, see Figure 8. 


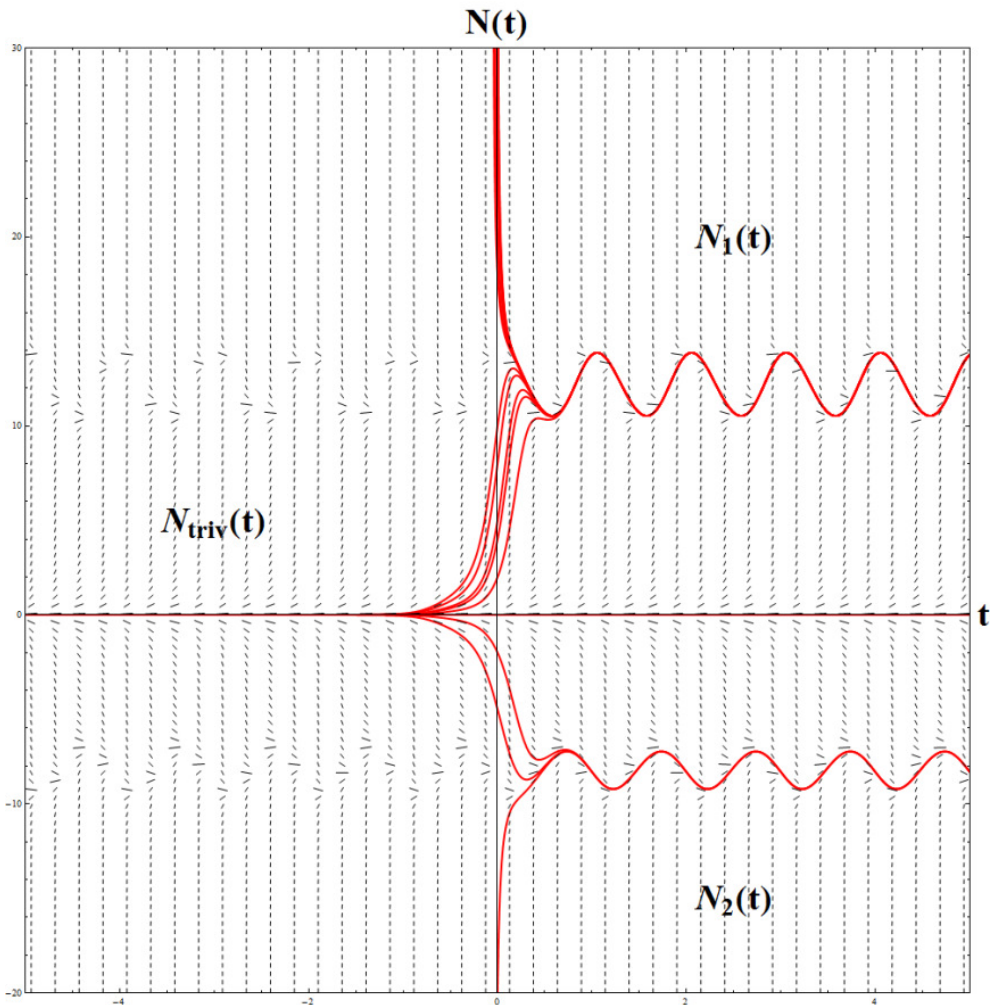

Figure 8: Existence of one positive asymptotically stable periodic solution of Eq. (4.13) in case of weak Allee effect for $\gamma_{1}=-1$ and stocking for $\gamma_{2}=-1$. 


\section{Example 4.10}

a. Consider the following differential equation with $A(t)=0$ and $h(t)>0$

$$
\begin{aligned}
\dot{N} & =(30+\sin 2 \pi t) N \\
& \times\left(1-\frac{N}{40+\cos 2 \pi t}\right)\left(\frac{N}{40+\cos 2 \pi t}\right)-\gamma_{2}(2+\cos 2 \pi t) N
\end{aligned}
$$

where $r(t)=30+\sin 2 \pi t, K(t)=40+\cos 2 \pi t, A(t)=0, h(t)=\gamma_{2}(2+\cos 2 \pi t)$.

b. Consider the following differential equation with $A(t)=0$ and $h(t)<0$

$$
\begin{aligned}
\dot{N} & =(20+\sin 2 \pi t) N \\
& \times\left(1-\frac{N}{25+\cos 2 \pi t}\right)\left(\frac{N}{25+\cos 2 \pi t}\right)-\gamma_{2}(16+\cos 2 \pi t) N
\end{aligned}
$$

where $r(t)=20+\sin 2 \pi t, K(t)=25+\cos 2 \pi t, A(t)=0, h(t)=\gamma_{2}(16+\cos 2 \pi t)$.

a. Let $\gamma_{1}=0$, and $\gamma_{2}=1$. The condition (4.1) is satisfied $A_{\max }=0 \leq K_{\min }=41$. By the Theorem (4.5), the condition

$$
\begin{aligned}
& h_{\max }<\frac{1}{4} r_{\min }\left(\frac{K_{\min }}{K_{\max }}\right)^{2} \\
& 3<\frac{1}{4}(29)\left(\frac{39}{41}\right)^{2}=6.559934
\end{aligned}
$$

is satisfied. Therefore, Eq. (4.14) has three periodic solutions: the asymptotically stable trivial solution $N_{\text {triv }}(t)=0$ and two positive solutions, asymptotically stable solution $N_{1}(t)$ and an unstable solution $N_{2}(t)$.

By the Theorem (4.5), these three solutions satisfy the inequality, 


$$
\begin{aligned}
& -\frac{K_{\min }}{2}<N_{\text {triv }}(t)<h_{\min }<N_{2}(t)<\frac{K_{\min }}{2}<N_{1}(t)<K_{\max } \\
& -19.5<N_{\text {triv }}(t)<1<N_{2}(t)<19.5<N_{1}(t)<41 .
\end{aligned}
$$

which are the corridors of these periodic solutions, see Figure 9(a).

b. Let $\gamma_{1}=0$, and $\gamma_{2}=-1$. The condition (4.1) is satisfied $A_{\max }=0 \leq K_{\min }=24$. By the Theorem (4.5), the condition

$$
\begin{aligned}
& h_{\max }<-\frac{3}{4} r_{\min }\left(\frac{K_{\min }}{K_{\max }}\right)^{2} \\
& -15<-\frac{3}{4}(19)\left(\frac{24}{26}\right)^{2}=-12.142012
\end{aligned}
$$

is satisfied. Therefore, Eq. (4.15) has three periodic solutions: the trivial solution $N_{\text {triv }}(t)=0$ which is unstable a positive solutions $N_{1}(t)$ and a negative solution $N_{2}(t)$, both asymptotically stable.

By the Theorem (4.5), these three solutions satisfy the inequality,

$$
\begin{gathered}
-K_{\max }<N_{2}(t)<-\frac{K_{\min }}{2}<N_{\text {triv }}(t)<K_{\min }<N_{1}(t)<2 K_{\max } . \\
-24<N_{2}(t)<-12<N_{\text {triv }}(t)<24<N_{1}(t)<52 .
\end{gathered}
$$

which are the corridors of these periodic solutions, see Figure 9(b). 


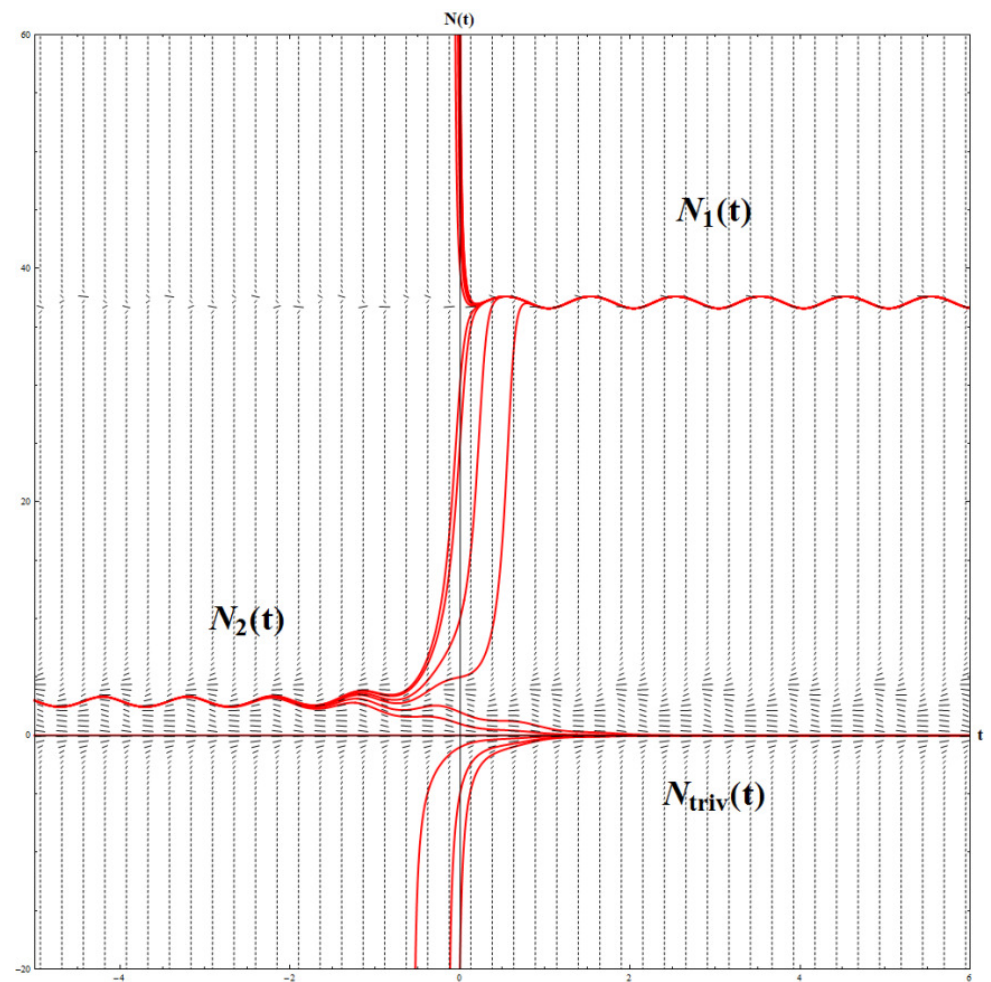

Figure 9(a): Existence of one positive asymptotically stable and one positive unstable periodic solution of Eq. (4.14) in case of harvesting for $\gamma_{1}=0, \gamma_{2}=1$.

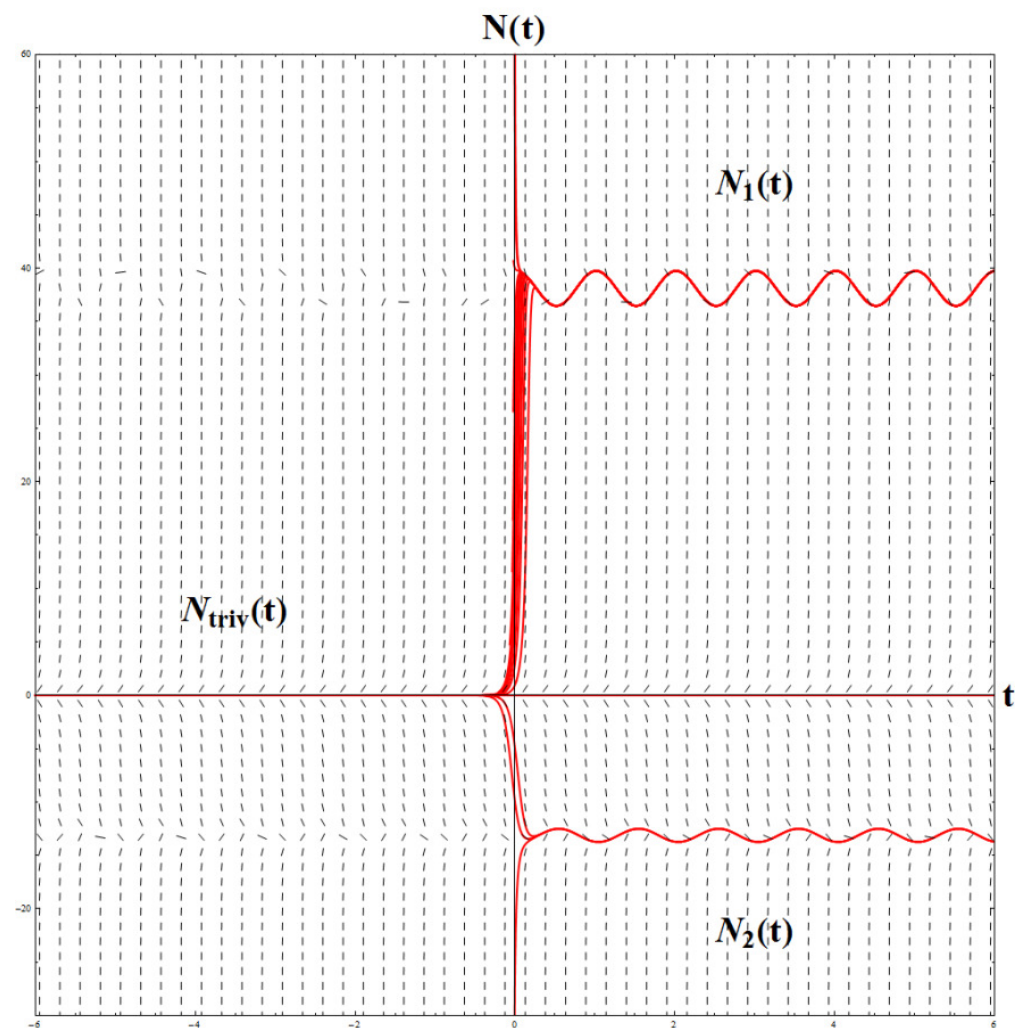

Figure 9(b): Existence of one positive asymptotically stable of Eq. (4.15) in case of stocking $\gamma_{1}=0, \gamma_{2}=-1$. 


\section{Chapter 5}

\section{CONCLUSION}

In this thesis, we have established the following three patterns in the dynamics of Eq. (1.1) in relation to the changes of the Allee threshold $A(t)$ :

a. for $A(t)>0$, a bistability is observed: an unstable positive periodic solution $N_{2}(t)$ is located between a pair of asymptotically stable solutions, a positive solution $N_{1}(t)$ and the trivial solution $N_{\text {triv }}(t)$; all solutions with positive initial values split into two groups that are attracted to one of the solutions in this pair solutions with initial data $N_{0}<A_{\min }$ and $N_{0}>A_{\max }$ (more precisely, with $N_{0}<N_{2}\left(t_{0}\right)$ and $\left.N_{0}>N_{2}\left(t_{0}\right)\right)$ approach, respectively, $N_{\text {triv }}(t)$ and $N_{1}(t)$ as $t \rightarrow+\infty$

b. for $A(t)=0$, the semi-stable trivial solution $N_{\text {triv }}(t)$ does not attract solutions with positive initial values anymore, but still attracts those with negative initial values; an unstable positive periodic solution $N_{2}(t)$ disappears, whereas the only remaining positive periodic solution $N_{1}(t)$ attracts all solutions with positive initial data;

c. for $A(t)<0$, the trivial solution $N_{\text {triv }}(t)$ completely loses stability and becomes a repellor, one of the nontrivial periodic solutions, $N_{2}(t)$, emerges again, this time as a negative asymptotically stable solution, whereas the status of a positive 
asymptotically stable periodic solution $N_{1}(t)$ does not change: as in (b), it attracts all solutions with positive initial data.

Clearly, the dynamics in (a) is the most complicated; in (b) and (c) the first quadrant is invariant - all solutions with positive initial data remain there and are eventually attracted by the only positive periodic solution $N_{1}(t)$. Furthermore, in all three cases, solutions with large positive and negative (rather large negative) initial values blow up backward in time. However, no troubles occur for $t \geq t_{0}$. The transition dynamics for Eq. (3.21) associated with the variation of parameter $\gamma$ from 1 to -1 with the step -0.25 is summarized in Figure 10. One can observe how the qualitative properties change from the bistability in case $\gamma>0$ to the situation where the asymptotically stable solution $N_{1}(t)$ attracts all solutions with positive initial values for $\gamma \leq 0$.

Taking into account that Eq. (1.1) models evolution of a single unstructured species in a fluctuating habitat, one is particularly interested in existence of asymptotically stable positive periodic solutions. Our analysis demonstrates that such solution, denoted $N_{1}(t)$ in this thesis, exists in all three cases (a) - (c); the basin of attraction in (a) is $\left(N_{2}\left(t_{0}\right),+\infty\right)$ and $(0,+\infty)$ for (b) and (c). 

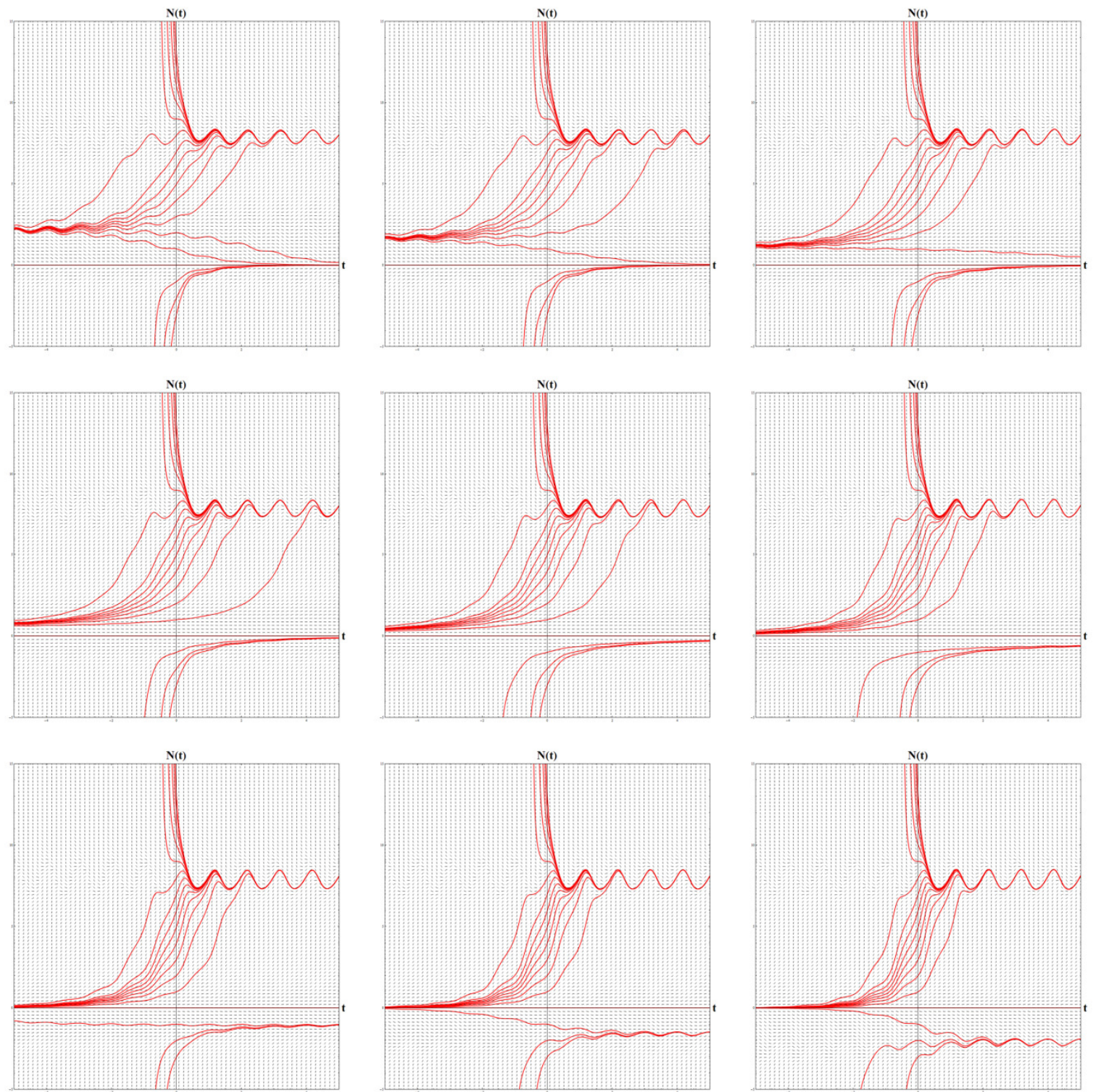

Figure 10: Qualitative changes in the dynamics of Eq. (3.21) as $\gamma$ changes from 1 to -1 with the step -0.25 .

In this thesis, we also discuss the effect of harvesting on a single species population in a fluctuating environment whose dynamics is described by the Eq. (1.7). We have determined the following five patterns in the dynamics of Eq. (1.7) in a relation to the changes of the Allee threshold $A(t)$ and harvesting $h(t)$;

a. for $A(t)>0$ and $h(t)>0$, and harvesting satisfies the condition $h_{\min }<\frac{r_{\min }}{K_{\max }^{2}}\left(\frac{K_{\min }-A_{\max }}{2}\right)^{2}$ then, Eq. (1.7) has three periodic solutions; the 
asymptotically stable trivial solution $N_{\text {triv }}(t)$ and two positive solutions, asymptotically stable solution $N_{1}(t)$ and an unstable solution $N_{2}(t)$. An unstable positive periodic solution $N_{2}(t)$ is situated between a pair of asymptotically stable solutions, a positive solution $N_{1}(t)$ and the trivial solution $N_{\text {triv }}(t)$; all solutions with positive initial values split into two groups that are attracted to one of the solutions in this pair - solutions with initial data $N_{0}<A_{\min }$ and $N_{0}>A_{\max }$ approach, $N_{\text {triv }}(t)$ and $N_{1}(t)$, respectively as $t \rightarrow+\infty$;

b. for $A(t)>0$ and $h(t)<0$ and harvesting satisfies the condition

i) $h_{\min }>-\frac{1}{4} r_{\min }\left(\frac{A_{\min }}{K_{\max }}\right)^{2}$ then Eq. (1.7) has three periodic solutions; the asymptotically stable trivial solution $N_{\text {triv }}(t)$ and two positive solutions, asymptotically stable solution $N_{1}(t)$ and an unstable solution $N_{2}(t)$, whereas the status of these three periodic solutions do not change, as in (a).

ii) $-r_{\min }<h_{\min }<h_{\max }<-\left(r_{\max }\right)\left(\frac{A_{\text {max }}+r_{\max }}{K_{\text {min }}}\right)^{2}$ then Eq. (1.7) has three periodic solutions; the trivial solution $N_{\text {triv }}(t)$ which is unstable a positive solutions $N_{1}(t)$ and a negative solution $N_{2}(t)$, both asymptotically stable. The trivial solution $N_{\text {triv }}(t)$ completely loses stability and becomes a repellor, one of the nontrivial periodic solutions, $N_{2}(t)$, arises again, this time as a negative asymptotically stable solution, whereas the status of a positive asymptotically stable periodic solution $N_{1}(t)$ does not change: as in b(i), it attracts all solutions with positive initial data. 
c. for $A(t)<0$ and $h(t)>0$ and harvesting satisfies the condition

i) $\max _{t \geq 0}\left\{-\frac{A_{\min } r_{\min }}{K_{\max }}, \frac{3}{4} r_{\min }\left(\frac{A_{\max }}{K_{\max }}\right)^{2}\right\}<h_{\min }<h_{\max }<\frac{1}{4} r_{\min }\left(\frac{K_{\min }}{K_{\max }}\right)^{2}$ then Eq. (1.7) has three periodic solutions; the asymptotically stable trivial solution $N_{\text {triv }}(t)$ and two positive solutions, asymptotically stable solution $N_{1}(t)$ and an unstable solution $N_{2}(t)$, whereas the status of these three periodic solutions are same as in (a).

ii) $h_{\max }<\min _{t \geq 0}\left\{-\frac{A_{\max } r_{\max }}{K_{\min }}, \frac{3}{4} r_{\min }\left(\frac{A_{\max }}{K_{\max }}\right)^{2}\right\}$ then Eq. (1.7) has three periodic solutions; the trivial solution $N_{\text {triv }}(t)$ which is unstable a positive solutions $N_{1}(t)$ and a negative solution $N_{2}(t)$, both asymptotically stable, whereas the status of these three periodic solutions are same as in b(ii).

d. for $A(t)<0$ and $h(t)<0$ and harvesting satisfies the condition

$h_{\max }>\max _{t \geq 0}\left\{-r_{\min },-4 r_{\min }\left(\frac{K_{\min }}{K_{\max }}\right)^{2}\right\}$ then Eq. (1.7) has three periodic solutions; ; the trivial solution $N_{\text {triv }}(t)$ which is unstable a positive solutions $N_{1}(t)$ and a negative solution $N_{2}(t)$, both asymptotically stable which are not change as in b(ii).

e. i) for $A(t)=0$ and $h(t)>0$ and harvesting satisfies the condition $h_{\max }<\frac{1}{4} r_{\min }\left(\frac{K_{\min }}{K_{\max }}\right)^{2}$ then Eq. (1.7) has three periodic solutions; the asymptotically stable trivial solution $N_{\text {triv }}(t)$ and two positive solutions, asymptotically stable 
solution $N_{1}(t)$ and an unstable solution $N_{2}(t)$, whereas the status of the periodic solutions are same as in (a).

ii) for $A(t)=0$ and $h(t)<0$ and harvesting satisfies the condition

$h_{\max }<-\frac{3}{4} r_{\min }\left(\frac{K_{\min }}{K_{\max }}\right)^{2}$ then Eq. (1.7) has three periodic solutions; the trivial solution $N_{\text {triv }}(t)$ which is unstable, a positive solutions $N_{1}(t)$ and a negative solution $N_{2}(t)$, both asymptotically stable whereas the status of the periodic solutions are same as in b(ii).

The changeover dynamics for Eq. (4.8) - Eq. (4.15) associated with the variation of parameter $\gamma_{1}$ and $\gamma_{2}$ from 1 to -1 with the step -0.25 is summarized in Figure 11. We observe that qualitative properties of the periodic solutions for the cases $\gamma_{1}, \gamma_{2}>0, \quad \gamma_{1}>0, \gamma_{2}<0, \quad \gamma_{1}<0, \gamma_{2}>0, \quad \gamma_{1}<0, \gamma_{2}<0, \quad \gamma_{1}=0, \gamma_{2}>0 \quad$ and $\gamma_{1}=0, \gamma_{2}<0$.

When we have a negative periodic solution of the differential equations (1.1) and (1.7) describing dynamics of a population, it has no biological meaning. Therefore, in these cases we can state the existence of a unique stable positive solution of these equations. Since it is stable, the population survives and fluctuates periodically. 

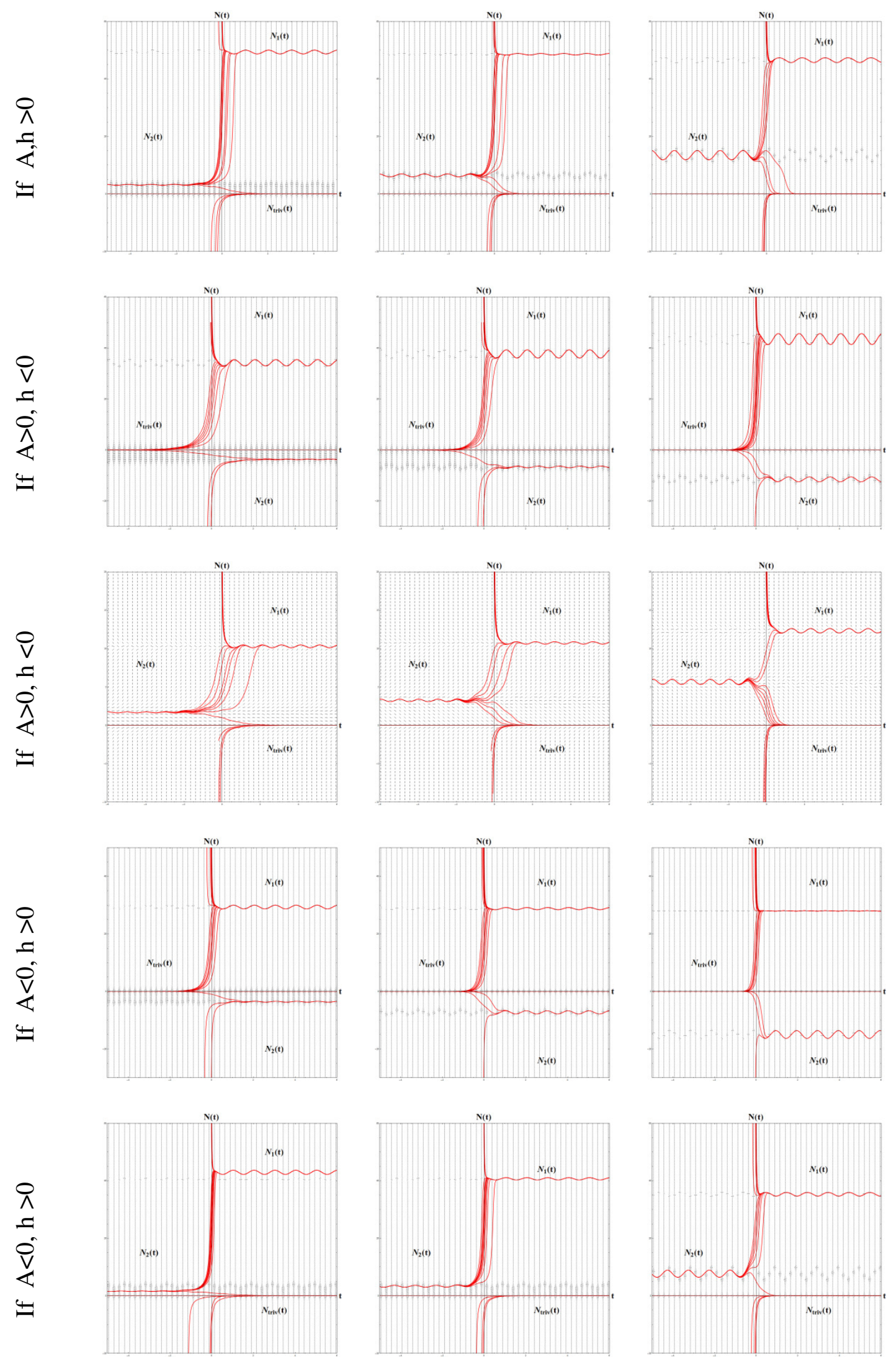

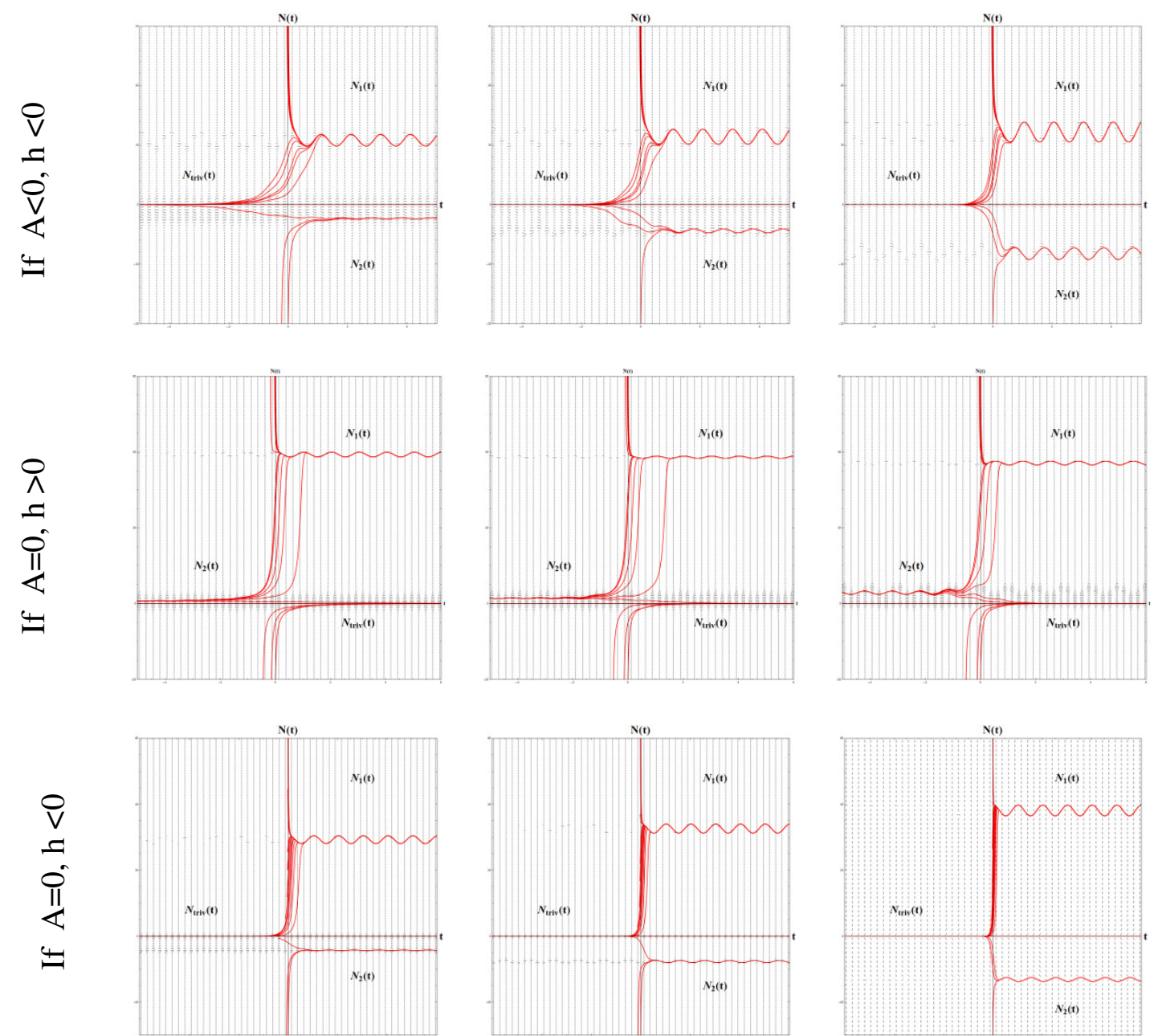

Figure 11: Qualitative changes in the dynamics of Eqs. (4.8) - (4.15) as $\gamma_{1}$ and $\gamma_{2}$ change from 1 to -1 with the step -0.25 . 
Table 1: Phenomenological models of demographic Allee effect and harvesting (or stocking) for the per capita growth rate $g(N)=r\left(1-\frac{N}{K}\right)\left(\frac{N}{K}-\frac{A}{K}\right)$. The meaning of the abbreviations are AS: Asymptotically Stable, SS: Semi-Stable, US: Unstable.

\begin{tabular}{|c|c|c|c|}
\hline $\begin{array}{l}\text { Continuous time } \\
\text { model }\end{array}$ & $\begin{array}{l}\text { Types of Allee } \\
\text { effect }(A(t))\end{array}$ & $\begin{array}{c}\text { Types of } \\
\text { harvesting }(h(t))\end{array}$ & Behaviour Stability \\
\hline \multirow[t]{3}{*}{$\frac{d N}{d t}=N g(N)$} & $\begin{array}{c}A(t)>0 \\
\text { (Strong Allee effect) }\end{array}$ & $h(t)=0$ & $\begin{array}{l}\text { Eq. (1.1) has three periodic solutions, } N_{t r i v}(t) \text { is AS, } \\
N_{1}(t)>0 \text { is AS and } N_{2}(t)>0 \text { is US. }\end{array}$ \\
\hline & $\begin{array}{l}\quad A(t)=0 \\
\text { (Weak Allee effect) }\end{array}$ & $h(t)=0$ & $\begin{array}{l}\text { Eq. (1.1) has two periodic solutions, } N_{\text {triv }}(t) \text { is } \mathrm{SS}, N_{1}(t)>0 \\
\text { is AS. }\end{array}$ \\
\hline & $\begin{array}{c}\quad A(t)<0 \\
\text { (Weak Allee effect) }\end{array}$ & $h(t)=0$ & $\begin{array}{l}\text { Eq. (1.1) has three periodic solutions, } N_{t r i v}(t) \text { is US, } \\
N_{1}(t)>0 \text { and } N_{2}(t)<0 \text { are AS. }\end{array}$ \\
\hline$\frac{d N}{d t}=N g(N)-h(t) N$ & $\begin{array}{c}A(t)>0 \\
\text { (Strong Allee effect) }\end{array}$ & $\begin{array}{c}h(t)>0 \\
\text { (harvesting) }\end{array}$ & $\begin{array}{l}\text { Eq. (1.7) has three periodic solutions, } N_{t r i v}(t) \text { is AS, } \\
N_{1}(t)>0 \text { is AS and } N_{2}(t)>0 \text { are US. }\end{array}$ \\
\hline
\end{tabular}




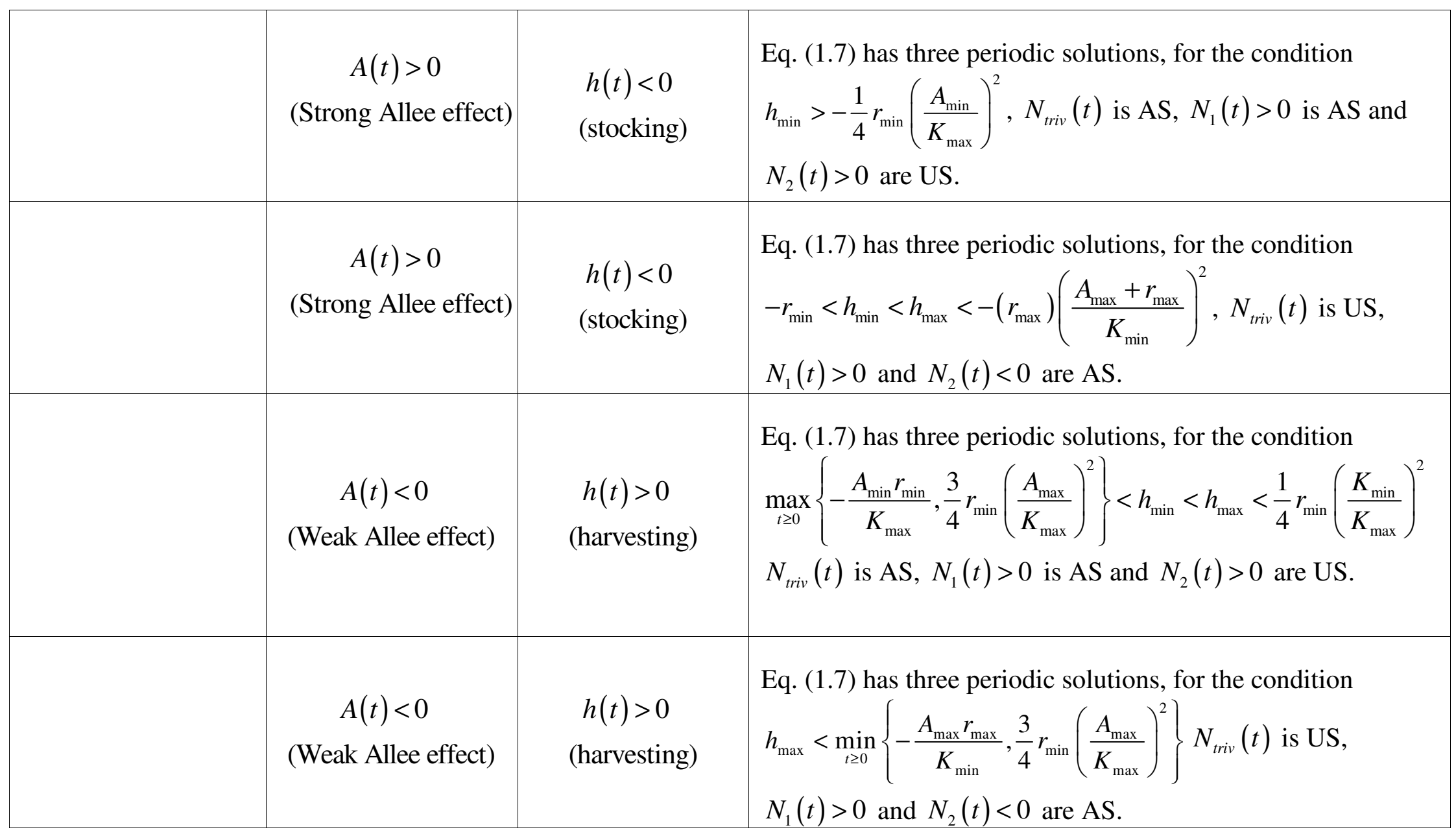




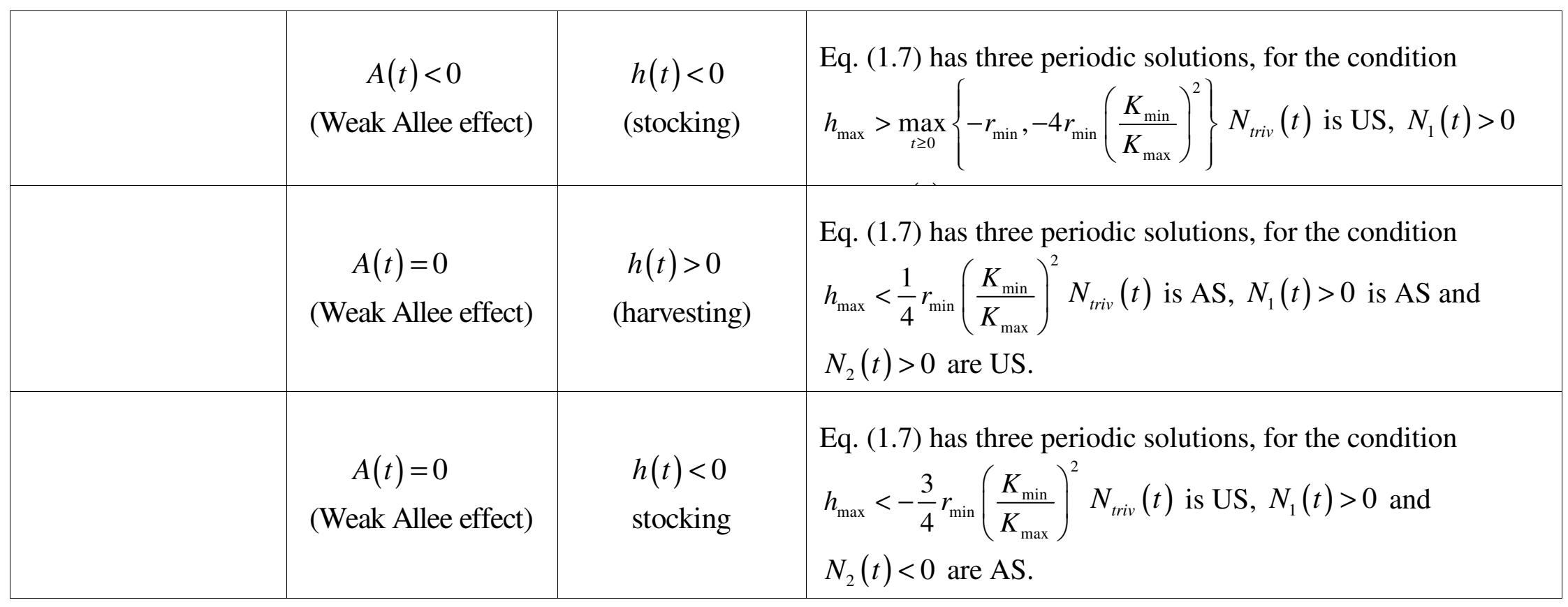




\section{REFERENCES}

[1] Nicholson, A.J. (1954). An outline of the dynamics of animal populations, Aust. J. Zool. 2, 9-65.

[2] Vance, R.R., \& Coddington, E.A. (1989). A nonautonomous model of population growth, J. Math. Biol. 27, 491-506.

[3] Henson, S.M., \& Cushing, J.M. (1997). The effect of periodic habitat fluctuations on a nonlinear insect population model, J. Math. Biol. 36, 201226.

[4] Brauer, F., \& Sánchez, D.A. (2003). Periodic environments and periodic harvesting, Nat. Resour. Model. 16, 233-244.

[5] Rogovchenko, S.P., \& Rogovchenko, Yu.V. (2009). Effect of periodic environmental fluctuations on the Pearl-Verhulst model, Chaos Soliton. Fract. 39, 1169-1181.

[6] Amarasekare, P. (1998). Allee effects in metapopulation dynamics, Am. Nat. 152, 298-302. 
[7] Lewis, M.A., \& Kareiva, P. (1993). Allee dynamics and the spread of invading organisms, Theoretical Population Biology. 43, 141-158.

[8] Schering, I. (1999). Allee effect increases the dynamical stability of populations, J. Theor. Biol. 199, 407-414.

[9] Fowler, M.S., \& Ruxton, G.D. (2002). Population dynamic consequences of Allee effects, J. Theor. Biol. 215, 39-46.

[10] Courchamp, F., Clutton-Brock, \& Grenfell, T. B. (1999). Inverse density dependence and the Allee effect, TREE. 14, 405-410.

[11] Courchamp, F., Berec, L., \& Gascoigne, J. (2008). Allee Effects in Ecology and Conservation, Oxford University Press Inc., New York.

[12] Boukal, D.S., \& Berec, L. (2002). Single-species models of the Allee effect: extinction boundaries, sex ratios and mate encounters, J. Theor. Biol. 218, 375-394.

[13] Berec, L., Angulo, E., \& Courchamp, F. (2006). Multiple Allee effects and population management, TREE. 22, 185-191. 
[14] Padhi, S., Srinivasu, P.D.N., \& Kiran Kumar, G. (2010). Periodic solutions for an equation governing dynamics of a renewable resource subjected to Allee effects, Nonlinear Anal. Real World Appl. 11, 2610-2618.

[15] Leggett, R.W., \& Williams, L.R. (1979). Multiple positive fixed points of nonlinear operators on ordered Banach spaces, Indiana Univ. Math. J. 28, 673-688.

[16] Langa, J.A., \& Robinson, J.C. A. (2002). Suárez, Stability, instability, and bifurcation phenomena in non-autonomous differential equations, Nonlinearity. 15, 887-903.

[17] Berger, A., \& Siegmund, S. (2008). Uniformly attracting solutions of nonautonomous differential equations, Nonlinear Anal. 68, 3789-3811.

[18] Hubbard, J.H., \& West, B.H. (1995). Differential Equations: A Dynamical Systems Approach, Part I: Ordinary Differential Equations, second corrected ed., Springer-Verlag, New York.

[19] Massera, J. (1950). The existence of periodic solutions of systems of differential equations, Duke Math. J. 17, 457-475.

[20] Corduneanu, C. (1977). Principles of Differential and Integral Equations, AMS Chelsea Publishing, The Bronx, New York, 
[21] Hale, J.K., \& Koçak, H. (1996). Dynamics and Bifurcations, third corrected ed., Springer-Verlag, New York.

[22] Pliss, V.A. (1966). Nonlocal Problems of the Theory of Oscillations, Academic Press, New York - London.

[23] Friedrichs, K.O. (1966). Advanced Ordinary Differential Equations, Gordon \& Breach, New York.

[24] Sánchez, D.A. (2002). Ordinary Differential Equations: A Brief Eclectic Tour, Mathematical Association of America, Washington.

[25] Nkashama, M.N. (1989). A generalized upper and lower solutions method and multiplicity results for nonlinear first-order ordinary differential equations, J. Math. Anal. Appl. 140, 381-395.

[26] Lakshmikantham, V., \& Leela, S. (1991). Existence and monotone method for periodic solutions of first-order differential equations, J. Math. Anal. Appl. 159, 127-146.

[27] Andersen, K.M., \& Sandqvist, A. (1991). On the number of closed solutions to an equation $\dot{x}=f(t, x)$, where $f_{x^{n}}(t, x) \geq 0 \quad(n=1,2$, or 3$)$, J. Math. Anal. Appl. 159, 127-146. 
[28] Korman, P., \& Ouyang, T. (1995). Exact multiplicity results for two classes of periodic equations, J. Math. Anal. Appl. 194, 763-779.

[29] Lins Neto, A. (1980). On the number of solutions of the equation $\frac{d x}{d t}=\sum_{j=0}^{n} a_{j}(t) x^{j}, \quad 0 \leq t \leq 1$, for which $x(0)=x(1)$, Invent. Math. 59, $67-76$.

[30] Alvarez, C., \& Lazer, A.C. (1986). An application of topological degree to the periodic competing species problem, J. Austral. Math. Soc. Ser. B. 28, 202-219.

[31] Ahmad, S., \& Montes de Oca, F. (1998). Extinction in nonautonomous $T$ periodic competitive Lotka-Volterra system, Appl. Math. Comput. 90, 155166.

[32] Hasanbulli, M., Rogovchenko, S.P., \& Rogovchenko, Yu.V. (2010). Dynamics of a single species in periodic environment under periodic harvesting, Preprint.

[33] Bardi, M. (1983). An equation of growth of a single species with realistic dependence on crowding and seasonal factors, J. Math. Biology. 17, 33-43. 
[34] Benardete, D.M., Noonburg, V.W., \& Pollina, B. (2008). Qualitative tools for studying periodic solutions and bifurcations as applied to the periodically harvested logistic equation. Amer. Math. Monthly. 115, 202-219.

[35] Campbell, D., \& Kaplan S.R. (2000). A bifurcation problem in differential equations. Math. Mag. 73, 194-203.

[36] Castilho, C., \& Srinivasu, P.D.N. (2007). Bio-economics of a renewable resource in a seasonally varying environment. Math. Biosci. 205, 1-18.

[37] Fan, M., \& Wang K. (1998). Optimal harvesting policy for single population with periodic coefficients. Math. Biosci. 152, 165-77.

[38] Lazer, A. (1980). Qualitative studies of the solutions of the equation of population growth with harvesting. Mat. Ensen. Univ. 17, 29-39.

[39] Lazer, A.C., \& Sánchez, D.A. (1975). Constant rate population harvesting equilibrium and stability. Theor. Pop. Biol. 8, 12-30.

[40] Lazer, A.C., \& Sánchez, D.A. (1984). Periodic equilibria under periodic harvesting. Math. Mag. 57, 156-158.

[41] Liu, P., Shi, J., \& Wang, Y. (2010). Periodic solutions of logistic type population model with harvesting. J. Math. Anal. Appl. 369, 730-735. 
[42] Rizaner, F.B., \& Rogovchenko, S.P. (2012). Dynamics of a single species under periodic habitat fluctuations and Allee effect. Nonlinear Analysis. 13, $141-157$.

[43] Rizaner, F.B., \& Rogovcenko S.P. (2012). Harvesting in a Periodic Habitat with Allee Effect. International Journal of Applied Physics and Mathematics. 2, 175-178.

[44] Hasanbulli, M., Rizaner, F.B., Rogovcenko, S.P., \& Rogovchenko, Y., (2011). Dynamics of a single species in a fluctuating environment. $3^{\text {rd }}$ Swedish Meeting on Mathematics in Biology. Mathematics and Mathematical Statistics, Umeå University, Sweden. 